I NTER NATIONAL MONETARY FUND

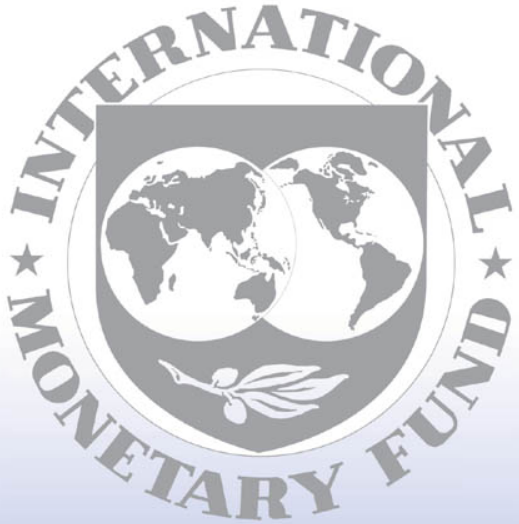

Staff

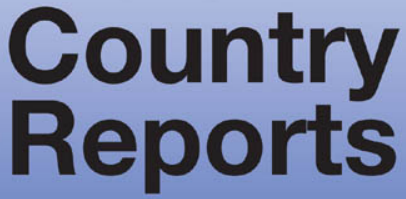


- 1995 International Monetary Fund

June 1995

IMF Staff Country Report No. 95/45

\section{Antigua and Barbuda- Recent Economic Developments}

This report on recent economic developments in Antigua and Barbuda was prepared by a staff team of the International Monetary Fund as background documentation for the periodic consultation with this member country. In releasing this document for public use, confidential material may have been removed at the request of the member.

Copies of this report are available to the public from

International Monetary Fund - Publication Services

700 19th Street, N.W. - Washington, D.C. 20431

Telephone: (202) 623-7430 - Telefax: (202) 623-7201

Telex (RCA): 248331 IMF UR

Internet: publications@imf.org

Price: $\$ 15.00$ a copy

International Monetary Fund

Washington, D.C. 
This page intentionally left blank

CInternational Monetary Fund. Not for Redistribution 


\section{INTERNATIONAL MONETARY FUND \\ ANTIGUA AND BARBUDA \\ Recent Economic Developments}

Prepared by a staff mission consisting of Humberto Arbulu-Neira (Head), Andres Gluski, Wayne Lewis, Ewe-Ghee Lim, and Ricardo Velloso (all WHD)

Approved by the Western Hemisphere Department

April 11, 1995

\section{Contents}

Basic Data iv-v

I. Overview 1

II. Macroeconomic Trends 2

1. Background

2. Expenditure and savings

3. Production by sectors a. Tourism

b. Construction

c. Agriculture and manufacturing

4. Prices, wages, and employment

$\frac{2}{2}$
$\frac{2}{2}$
$\frac{3}{3}$
$\frac{3}{3}$

III. Public Finances 4

1. Overall developments

2. Central Government

a. Revenue and grants

b. Expenditure and net lending

3. Rest of the public sector

a. Social Security Scheme and

Medical Benefits Scheme

b. Public enterprises

IV. Financial Sector

1. Institutional framework

2. Overall developments in the banking system 10

\begin{tabular}{ll}
$3 . \quad$ ECCB operations & 10 \\
\hline
\end{tabular}

a. Operations in Antigua and Barbuda $r$

b. ECCB policy developments 11

4. Commercial banks 11

\begin{tabular}{ll}
\hline . Interest rates & 12 \\
\hline
\end{tabular}

CInternational Monetary Fund. Not for Redistribution 
V. External Sector

1. Balance of payments

a. Overall developments 12

\begin{tabular}{ll}
\hline b. Merchandise trade & 12 \\
\hline
\end{tabular}

\begin{tabular}{ll}
\hline c. Tourism & 13 \\
\hline
\end{tabular}

\begin{tabular}{lll}
\hline d. & Other services and transfers & 14
\end{tabular}

\begin{tabular}{ll}
\hline e. Capital account & 14
\end{tabular}

2. External debt 14

3. Exchange and trade system 15

a. Exchange rate developments 15

b. Trade system 16

Text Tables

1. Selected Economic Indicators 17

2. Summary Operations of the Consolidated Public Sector 18

3. Summary Operations of the Central Government 19

4. Central Government Revenue and Grants 20

5. Summary Operations of the Rest of the General Government 21

6. Summary Operations of the Consolidated Public Enterprises 22

7 . Monetary Survey 23

8. Eastern Caribbean Central Bank Operations in Antigua and Barbuda

9. Summary Accounts of Commercial Banks 25

10. Balance of Payments 26

11. Public and Publicly Guaranteed External Debt 27

\section{Appendices}

I. Summary of Fiscal Measures 28

II. Pax Summary 30

III. Earmarking of Government Revenue to
Service Selected Loans

$\underline{\text { Statistical Appendix Tables }}$

12. GDP by Sector of Origin at Current Prices 38

13. GDP by Sector of Origin at Constant 1977 Prices 39

14. Expenditure on GDP at Current Prices 4

15. Expenditure on GDP at Constant 1985 Prices $r$

\begin{tabular}{lll}
\hline 16. & Savings and Investment & 42 \\
\hline
\end{tabular}

17. Indicators of Construction Activity 4

18. Electricity Generation, Consumption, and Tariffs 44

\begin{tabular}{lll}
\hline 19. & Consumer Prices & 45 \\
\hline
\end{tabular}

20. Retail Prices of Petroleum Products 4

21. Estimates of Population and Labor Force 4

22. Operations of the Consolidated Public Sector 48 
23. Central Government Operations 55

24. Central Government Revenue and Grants 57

25. Central Government Expenditure $r$

26. Commercial Banks' Operations 59

27. Distribution of Commercial Bank Credit to Private Sector 60

28. Selected Interest Rates 61

29. Balance of Payments 62

\begin{tabular}{lll}
\hline 30. & Selected Tourism Statistics & 64
\end{tabular}

31. Air Stayover Arrivals $\quad 65$

32. Air Stayover Arrivals by Origin $\quad 66$

33. Tourism Capacity 167

34. Visitor Arrivals as a Share of the Caribbean Market 68

35. Exchange Rates $\quad 69$

36. Terms of External Debt $r$

37. External Debt Excluding Interest Arrears 73

38. Summary of Arrears Outstanding on External Debt 1

Chart

1. Exchange Rate Developments $16 \mathrm{a}$

CInternational Monetary Fund. Not for Redistribution 
Population and related vital statistics

Population, 1994 (thousands)

Average annual rate of growth of population in 1987-92 (percent)

Population density (per sq kilometer)

Arable land (percent of land area)

Life expectancy at birth (years)

Infant mortality rate (per 1,000 live births)

Nutrition

Per capita protein intake (grams per day)

Bealth

Population per physician

Population per hospital bed

Population with access to health care (percent of total)

Immunization of 1 year olds against infectious diseases (percent)

\section{Education}

Adult literacy rate (percent)

Primary school enrollment (percentage of children, 5-9 years old)

GDP at market prices (1994 prel.)

SDR 344.6 million

GDP per capita (1994 prel.)

SDR 5,356

\section{Origin of GDP (in percent)}

Asriculture

Manufacturing, mining and quarrying

Hotels and restaurants

Construction

Iransport and communications

Government services

Banks and insurance

Trade

Housing

Electricity and water

Other services

Net indirect taxes

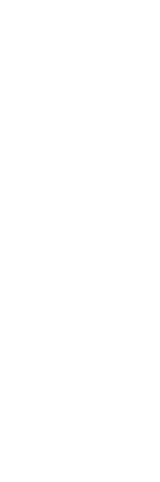

Ratios to GDP

Exports of goods and nonfactor services

Of which: tourism receipts

Imports of goods and nonfactor services

Current account of the balance of payments

(including grants)

Public sector revenue and grants

Public sector expenditure

Public sector savings

Public investment

Public sector overall surplus or deficit (-)

External public debt (end of year)

Money and quasi-money (end of year)

Gross national savings

Gross domestic investment

$\begin{array}{rr}1990 & 1991 \\ 101.7 & 102.9 \\ 75.5 & 72.9 \\ 106.9 & 105.4 \\ -14.8 & -9.4 \\ 25.4 & 24.1 \\ 29.7 & 29.6 \\ -2.7 & -3.3 \\ 2.9 & 3.4 \\ -4.3 & -5.5 \\ 83.2 & 83.6 \\ 52.6 & 56.4 \\ 12.6 & 15.7 \\ 27.4 & 25.2\end{array}$

Prel.

1994

3.2

3.3

12.3

8.0

16.8

14.6

6.5

8. 4

6.1

3.5

1.7

15.6

Prel.

1994

(percent)

110.4

76.3

110.0

$-7.7$

25.0

29.8

$-4.8$

0.9

$-4.8$

82.2

57.0

13.3

21.0

\section{$\underline{1993}$}

$\begin{array}{rr}111.6 & 109.6 \\ 82.7 & 84.2\end{array}$

$111.8 \quad 107.8$

$\begin{array}{ll}-6.9 & -5.4\end{array}$

$25.5 \quad 26.5$

$30.2 \quad 33.0$

$-3.3 \quad-4.0$

$2.4 \quad 2.4$

$-4.7 \quad-6.5$

$79.2 \quad 81.3$

$60.7 \quad 63.1$

$17.6 \quad 16.3$

$24.4 \quad 21.7$ 
Annual chanses in selected indicators

Real GDP at factor cost

GDF at curront market prices

GDP deflator

Real GDP per capita

Consumer prices (annual average)

Public sector rovenue and grants

Public sector expenditure and not londins

Monetary liabllities to the privete sector Money

Quas 1-money

Net domestic assets $1 /$

Credit to the public sector (net) 1/

Credit to the private sector $1 /$

Merchandise exports (in U.S. dollars)

Morchandise imports (in U.S. dollers)

\section{Public sector finances}

Revenue and grants

Expenditure and net lendine

Current account surplus or deficit (-)

Overall surplus or deficit (-)

External financing (net)

Domestic Inancing

\section{Balance of peyonts}

Morchandise exporta

Merchendise Imports

Investment incone (net)

Other services and transfers (net)

of which: tourlaw recelpta

Balance on current eccount (including srants)

Capital account

Official (net)

Banking system

Private direct investmont

Overall deficit (-)

Change in imputed reserves (1ncrease -)

Change in arrears (not)

Rescheduling; other financing

IMF data as of March 31. 1995

\section{Article VIII status}

Intervention currency and rate

Quota

Fund holdings of Eastern Caribbean dollars Total Fund holdings

Special Drawing Rights Department

Cumulative SDR allocation

Net ecquistion or utilization of SDRs

Holdings of SDR:

Irust Fund loan

Shares of profits from sold sales

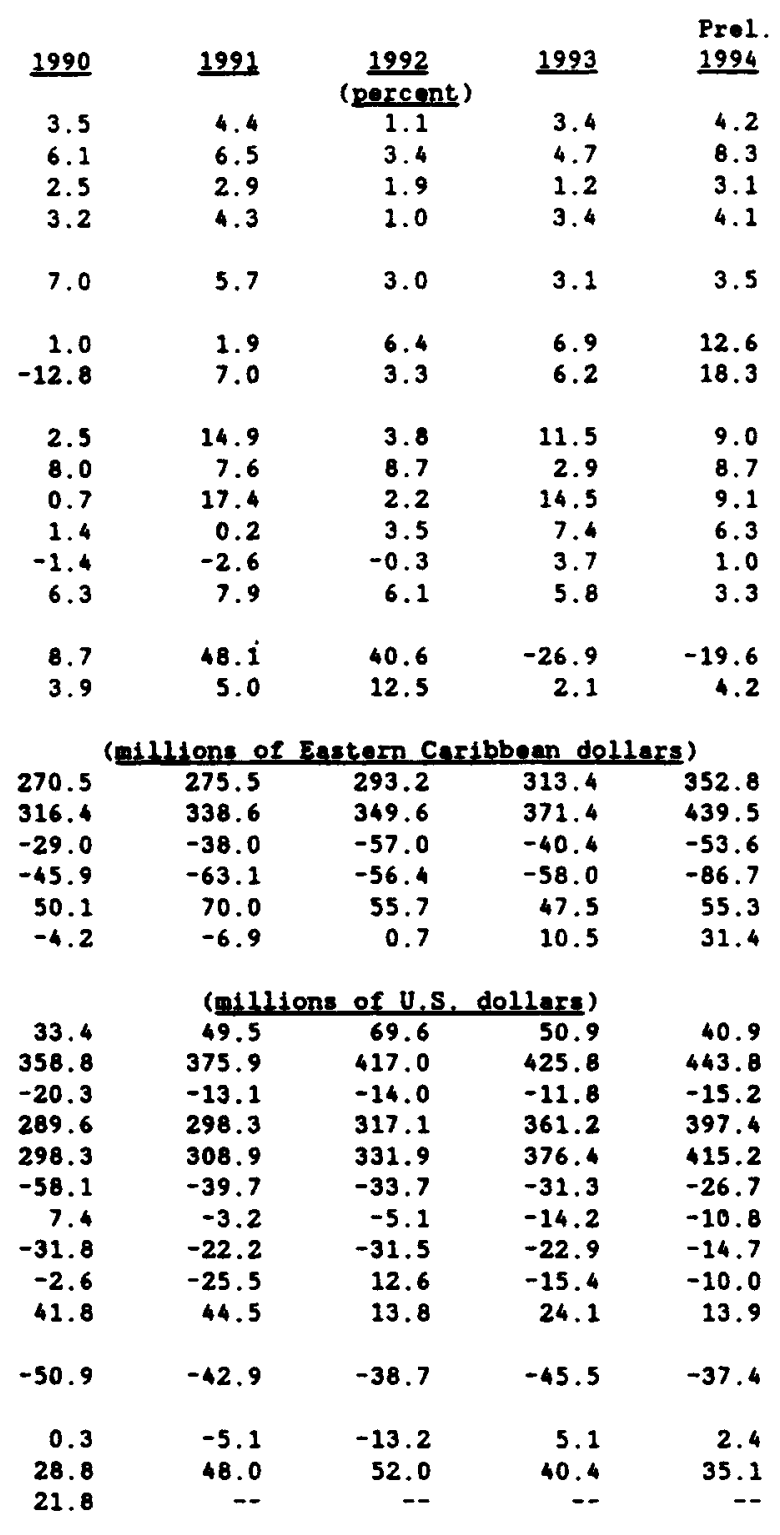

U.S. dollar at EC\$2.70 per US\$ SDR 8.5 million SDR 8.5 million 100 percent of quota

1/ Change in percent of liabilities to the private sector at the beginning of the period. 
This page intentionally left blank

CInternational Monetary Fund. Not for Redistribution 


\section{Overview}

A period of rapid economic growth in the 1980 s was followed by a slowdown in the early 1990s as the growth of tourism--the mainstay of the economy--and tourism-related construction activity slackened. The current balance of the public sector deteriorated substantially, mainly reflecting a sharp increase in wages and other current expenditure; public investment declined; and external arrears continued to build up.

The growth of real GDP picked up to about 4 percent in 1993-94 on the strength of increased tourism while inflation remained low $(3-31 / 2$ percent a year). The current account deficit of the public sector widened from less than 3 percent of GDP in 1990 to 4 percent of GDP in 1994, and the overall deficit went up to $61 / 2$ percent of GDP. Public investment, averaging $21 / 2$ percent of GDP in the period 1990-94, has remained too low to provide for the upgrading of infrastructure needed for the continued growth of tourism and the economy. In 1994 increased revenues were more than offset by an increase in current expenditure, including wages, associated in part with the March 1994 general election. In 1994 and early 1995 a series of revenue measures were introduced that include an education levy, higher contribution rates for medical benefits, increased taxation of tourism, and higher property taxes.

The main source of deficit financing in recent years has been the accumulation of external payments arrears (the buildup averaged 10 percent of GDP a year in the period 1990-94); there have been virtually no new external credits since 1991. In 1994 there was a significant amount of domestic financing. Banks and nonbank financial institutions extended credits subject to the earmarking of fiscal revenue to service their loans, and there was a further accumulation of arrears with the private sector.

The external current account deficit decreased from an average of $91 / 2$ percent of GDP in 1990-93 to $51 / 2$ percent of GDP in 1994 as a result of the continued strengthening of tourism and a slowdown in imports associated with a fall in investment. The capital account shifted to a deficit in this period and the overall balance of payments deficit, that averaged almost 10 percent of GDP in 1990-94, was financed mainly by an accumulation of arrears.

External debt, which has been about 80 percent of GDP since the beginning of the 1990s, consists largely of external arrears that amounted to 68 percent of GDP at the end of 1994. Most of the servicing of external debt that has taken place in recent years (2 percent of GDP on average) has resulted from the earmarking of public sector revenue for such payments.

In January 1995 Antigua and Barbuda implemented the first stage of the Caribbean Community's (CARICOM) common external tariff (CET). At the same time consumption taxes were raised to offset the fiscal impact of the reduction in the average tariff. 


\section{Macroeconomic Trends}

\section{Background}

Antigua and Barbuda is a small, open economy highly dependent on tourism. Tourism, as measured by the hotel and restaurant sector, accounts for some 12 percent of GDP; in addition, a significant part of the activity in the transport, trade, and construction sectors (accounting for an additional 27 percent of GDP) derives from tourism.

The growth of real GDP picked up to 4.2 percent in 1994 after slowing down to 3.2 percent a year in 1990-93 (from 7 percent a year in 1988-89) (Table 1). The growth in 1994 reflected the recovery of tourism and the short-run effect of expansionary fiscal policies prior to the March 1994 general election.

\section{Expenditure and savings}

Real domestic expenditure, after remaining essentially unchanged during the period 1990-9'2, rose by about 1 percent in 1993 when domestic investment increased sharply. In 1994 domestic expenditure rose by 1 1/2 percent in real terms, owing to a sharp increase in public consumption that offset decreases in private consumption and investment (Statistical Appendix Tables 14 and 15). Domestic savings increased from an average of 14 percent of GDP in 1990-92 to 17 1/2 percent in 1993; however, with the decline in investment (from $241 / 2$ percent of GDP in 1993 to $211 / 2$ percent in 1994, both domestic savings and the use of foreign savings decreased in that year (Statistical Appendix Table 16).

\section{Production by sectors}

\section{a. Tourism}

Economic activity in hotels and restaurants accounts for 12 percent of GDP, although as noted above this understates the dominant role of tourism in the economy of Antigua and Barbuda. In 1993 air arrivals increased by 14.3 percent, and real value added of tourism (hotels and restaurants) increased by 10 percent. This increase is partly as a result of greater marketing efforts, particularly in the European market. The growth of real value added in tourism slowed to 6 percent in 1994 as the strong increase in air arrivals from Europe was partly offset by a 3.4 percent decline in air arrivals from the United States. Also, the average tourist stay has lengthened as European tourists tend to stay longer than other visitors (Statistical Appendix Table 32). The number of rooms for tourist accommodation rose by an average of 3.2 percent a year in 1993-94, and the recovery of tourism resulted in an increase in room occupancy rates (Statistical Appendix Table 33). 


\section{b. Construction}

The share of construction in GDP fell from 9.5 percent in 1990 to 8 percent in 1994. Real value added in the construction sector stagnated in 1990-93 and increased by 4 percent in 1994 (but it remained lower than in the late 1980s). The 1994 increase resulted in part from public sector projects--including road improvements and public works in St. John's-assoclated with the March 1994 election campaign.

\section{c. Agriculture and manufacturing}

The agricultural sector accounts for only 3 percent of GDP. Production is directed to the local market, but none of the main crops is sufficient to meet domestic demand. Real value added in agriculture, after increasing by an annual average of 3.4 percent in 1990-93, declined by 1 percent in 1994 as a result of a prolonged drought. Longer term prospects for the agricultural sector are constrained by limited water supplies and labor shortages.

The share of manufacturing in GDP fell from 2.8 percent in 1990 to 2.0 percent in 1994. Manufacturing comprises enclave-type production for the export of handicrafts, small appliances and electronic components, and production for the domestic market, mainly beverages and construction materials. In 1993 real value added declined by 5 percent as a result of the closure of one mattress producer, but it grew 2 percent in 1994 following the opening of a local brewery in late 1993. In late 1994 Parliament approved the Free Trade and Processing Zone Act, providing tax holidays and other incentives, to firms engaged in the manufacture of goods for export. As of February 1995 no firms had yet been established under the terms of this Act.

\section{Prices, wages, and employment}

As a result of the discipline imposed on Antigua and Barbuda by its membership in the Eastern Caribbean monetary union, movements in domestic prices in recent years have largely reflected imported inflation. The Government administers price controls on about 30 food items--mostly imported goods--and on petroleum products, in most cases by setting limits on trade margins. 1/ According to an unofficial price index, consumer prices rose by 3 percent a year in $1992-93$ and by 3.5 percent in 1994 (Statistical Appendix Table 19). 2/

Wage contracts generally are negotiated for periods of three years, and private sector wages are freely determined. Private sector wage increases slowed down from $71 / 2$ percent in 1990 to 6 percent a year in 1992-93,

1 For several foodstuffs, wholesale margins are limited to 10 percent, and retail margins to 15 percent (see SM/93/241, Appendix I).

2) The authorities have been developing a consumer price index with assistance from STA. 
reflecting a slowdown in economic activity and in public sector wages. 1/ In 1994 the growth of private sector wages picked up to an estimated 7 percent, partly reflecting an upturn in the economy and a more rapid growth of government wages. The freezing of civil servant wages in 1991-92 was followed by an increase of 5 percent in 1993 and 8 percent in 1994. Half of the 1994 increase was paid in 1994; the other half was postponed to 1995.

Shortages of workers--common in the 1980s--were reduced in the first half of the 1990s when the slower growth of the economy eased the demand for labor, and the supply increased as a result of the Government's liberal immigration policy regarding workers. The number of immigrant workers has fluctuated around an average of 7,300 in 1990-94 (about 25 percent of the active labor force; Statistical Appendix Table 21). Public sector employment was unofficially estimated at 9,500 in 1994, or one third of the labor force. The growth of public sector employment, that averaged 2 percent a year during the period 1990-93, is estimated to have risen by some 5 percent in 1994 ( 6 percent in the Central Government, which accounts for four fifths of public sector employment).

\section{Public Finances 2/}

\section{Overall developments}

In 1990-94 Antigua and Barbuda's fiscal position continued the substantial deterioration that began in the mid-1980s, when a sharp decline in public savings and an accumulation of external arrears led to the curtailment of foreign financing and large cuts in public investment.

Public sector dissavings rose from an average of 3.5 percent in the period 1990-93 to 4 percent of GDP in 1994, as the growth in revenue failed to keep pace with the sharp increase in current expenditure, which rose from an average of $271 / 2$ percent of GDP in 1990-93 to almost 30 percent in 1994 (Table 2). With negative public savings and a lack of foreign financing, public capital outlays remained low, averaging 2.4 percent of GDP in 1990-94. As a consequence, the maintenance of infrastructure has been neglected and the environment has been affected adversely. Taking account of a slowdown in capital revenue and grants during the period, the public

1/ Fund staff estimates for private sector wages are based on collective agreements and employment data provided by the Labor Department, the Employers' Federation, and the Social Security Scheme.

2f The nonfinancial public sector in Antigua and Barbuda consists of the Central Government, the Social Security Scheme, the Medical Benefits Scheme, and public enterprises, the main ones being the Antigua Port Authority, the Antigua Public Utilities Authority, Deep Bay Development Company, Central Marketing Corporation, and St. John's Development Corporation. 
sector deficit rose from an average of 4.8 percent of GDP in $1990-93$ to 6.5 percent in 1994.1

The Government continued to be unable to service fully its external debt despite the earmarking of revenue to service some loans, and arrears continued to accumulate. External financing was limited during the period as a result of the external arrears problem. Significant domestic financing of the public sector arose in 1993 from commercial bank credit, and in 1994 from domestic arrears to the private sector and credit from the nonbank financial sector. Most of the credits carried interest rates of $11-13$ percent and were guaranteed by earmarked revenues through arrangements similar to those for servicing some external obligations. $2 /$

\section{Central Government}

The current deficit of the Central Government (including unpaid interest) increased from an average of 1.2 percent of GDP in the period 1990-93 to 2.4 percent in 1994 , owing mainly to an increase in the government wage bill and outlays on goods and services (Table 3). During the period 1990-93 capital revenue and grants averaged less than 1 percent of GDP, and capital expenditure was 1 imited to an average of about 2 percent of GDP, resulting in an overall deficit of 2.3 percent of GDP on average. In 1994 the deficit rose to an estimated 4.9 percent of GDP, partly reflecting an increase in capital expenditure and net lending.

In 1990-94 the central government deficits were financed mainly by the accumulation of external and domestic arrears. Arrears on domestic obligations comprise overdue contributions to the Social Security Scheme and Medical Benefits Scheme, overdue bills to domestic suppliers, and unpaid interest on treasury bills and bonds. The Government's net obligations to the Social Security Scheme and the Medical Benefits Scheme continued to rise in 1994 and amounted to about 14 percent of GDP at the end of the year (of which 11 percentage points of GDP represented overdue contributions), despite a transfer in 1994 of government land to the Social Security Scheme in partial settlement of contributions in arrears. Also, most of the financing from the domestic financial system in 1993-94 (mentioned above) was provided to the Central Government.

1) The overall balance of the consolidated public sector is estimated from the financing side. The financing items include: (i) external debt disbursements and repayments (accrual basis); (ii) changes in net liabilities to the banking system and the Eastern Caribbean Central Bank; (iii) accumulation of external arrears (interest and principal); and (iv) change in domestic arrears of the Central Government.

2/ Information on the earmarking of revenue to service selected external and domestic loans to the Government is presented in Appendix III. 


\section{a. Revenue and grants}

Central government revenue averaged 21.5 percent of GDP in 1990-94, and tax revenue averaged 17.4 percent of GDP in the same period, of which about two thirds is related to external transactions (Table 4). 1/ The buoyancy of the tax system is limited as shown by the fall since 1990 in corporate income taxes (in relation to GDP) because of inadequate collection procedures; the stagnation of property taxes owing to infrequent revision of property valuations; and the slow growth of customs duty revenue. The average import tariff declined in the first half of the 1990s, from an average of 4.5 percent of imports in 1990 to 3.9 percent in 1994 , mainly because of the high incidence of exemptions. Tariff exemptions are granted under laws that aim at fostering the development of the hotel sector and new manufacturing businesses and also on a discretionary basis to nonprofit organizations. Import duty exemptions, including those for public enterprises, amounted to an estimated 3 percent of GDP in 1993 (the most recent estimate available).

The decline in the average tariff also reflected deficient customs administration. To address this problem, Parliament approved the Customs Management and Control Act in January 1995, substantially increasing penalties for offenses including the under-invoicing and nondeclaration of imports.

A number of tax measures implemented in mid-1994 boosted revenue by one half of 1 percentage point of GDP in that year. The cruise-ship passenger tax was doubled to US\$6 per passenger; the customs service charge was doubled to 5 percent of the import value; and the sales tax on restaurants was extended to nonhotel establishments.

To address the fiscal imbalance the authorities are in the process of developing an adjustment program with help from the Eastern Caribbean Central Bank (ECCB), Caribbean Development Bank (CDB), and the Organization of Eastern Caribbean States (OECS). As part of the program, an education levy on wages and salaries was introduced at the beginning of 1995 at a basic rate of 2.5 percent (Appendix I). Also, indirect taxes on the tourism sector and several licenses and fees were increased. In addition, property taxes were raised substantially in early 1995 when the value of property used as a basis for taxation for the major part of St. John's (including all commercial properties) was nearly quadrupled on average, and tax rates were reduced by one half. In early 1995 the authorities were revising the cadastre for the remainder of St. John's. These tax measures are expected to increase revenue by about 1.8 percent of GDP in 1995 .

The Customs Duties Act of 1993, that took effect in January 1995 , implemented the first stage of CARICOM's CET for Antigua and Barbuda,

1 Consumption duties (which are mostly on imports), import duties, and tourism-related taxes accounted for 12 percent of GDP in 1994, compared with an overall tax ratio of 17.7 percent of GDP. 
resulting in a lower average tariff. I/ To avoid adverse fiscal effects, the authorities increased the consumption tax for goods subject to lower import tariffs as a consequence of the CET.

\section{b. Expenditure and net lending}

Total expenditure and net lending of the Central Government rose from 22-23 percent of GDP during the period 1990-93 to 26.3 percent in 1994 -owing to increases in the wage bill, other current expenditure on goods and services, and a loan to LIAT (the regional airline).

Despite the postponement until 1995 of the payment of one half of the 8 percent general wage increase, the government wage bill increased by 15 percent in 1994 (following a 5 percent increase in 1993), as government employment rose by about 6 percent and a vocational training scheme was introduced. Under the vocational training scheme, the Government pays a wage (and social security and medical benefit contributions) to individuals entering apprenticeships--mostly in private enterprises--for a period of 6-12 months. In 1994, expenditure under the scheme amounted to the equivalent of 0.5 percent of GDP.

A loan of EC\$10 million was granted by the Government to LIAT, the regional airline, as part of a financial rescue plan. The loan permitted the clearing of LIAT's arrears with the CDB, a step needed to proceed with the sale of the airline. In the meantime, Antigua and Barbuda, together with Trinidad and Tobago, took over the airline's administration.

\section{Rest of the public sector}

\section{a. Social Security Scheme and Medical Benefits Scheme}

The Social Security Scheme provides pensions and benefits including maternity, disability, sickness, and funeral expenses. It is funded by monthly contributions of 8 percent of employees' earnings ( 5 percent paid by the employer, 3 percent by the employee), up to a maximum monthly income that was raised from EC $\$ 2,500$ to EC $\$ 4,500$ in 1994. The Social Security Scheme had cash surpluses that averaged 2 percent of GDP in the period 1990-94, notwithstanding the Government's failure to pay contributions (Table 5). (Government employees comprise 45 percent of all contributors to social security, but in 1993-94 the Government paid neither the employer's contribution nor the employees' contributions that it had retained at source.) In 1994 an actuarial review of social security operations, based on 1993 data, recommended that the government arrears problem be addressed; otherwise the Social Security Scheme would begin to experience cash-flow shortages by 1998 . The Government's total obligations to the Social Security Scheme reached about EC\$150 million at end-1994, after taking

1 See SM/93/241, Appendix V. 
account of a government transfer of 100 acres of Crown land to settle EC\$5 million in arrears.

The Medical Benefits Scheme (MBS) provides benefits for the treatment of specific chronic diseases (diabetes, cancer, hypertension, etc.). The MBS recorded small cash surpluses that averaged 0.3 percent of GDP in the period 1990-94. In January 1995 the contribution was raised from 5 percent to 7 percent of employees' earnings (shared equally between employers and employees), and the celling on monthly earnings subject to contributions from EC $\$ 2,500$ to EC $\$ 4,500$.

As with the Social Security Scheme, in 1993-94 the Government failed also to pay the employer contribution and transfer the employee contribution retained at source, and liabilities to the MBS rose, from EC\$26 million at end-1993 to about EC\$30 million (2.3 percent of GDP) at end-1994. In addi tion, the MBS granted EC\$5 million to the Government to help meet the costs of a government-owned hospital and a number of health-care programs.

\section{b. Public enterprises}

The dissavings of the public enterprises averaged 4.7 percent of GDP in 1990-94 (Table 6). With little capital revenue or expenditure, the overall deficit also was about 5 percent of GDP during the period and was financed primarily by an accumulation of external arrears.

A large part of the public sector's external arrears are owed by the Deep Bay Development Company to the Italian export credit guarantee agency (more than 40 percent of total external arrears) on a loan for the construction of the Royal Antiguan Hotel. Since it began operations in 1988, the hotel has had management and maintenance problems that led to low occupancy rates and continuous financial losses. As a result, debt service has not been paid on the loan. In October 1994 a management contract with a multinational corporation was terminated and a 15-year contract (with an optionto-buy clause) was signed-with a domestic group, committing the new management to invest US\$4.8 million during the first year to refurbish the hotel. The management contract is expected to set the stage for the privatization of the hotel. However, since the market value of the hotel appears to be lower than the outstanding amount of the loan, the authorities have sought to refinance the debt with the Italian agency on terms that would include a substantial write-off.

The Antigua Public Utilities Authority (APUA) provides electricity, water, and telephone services. Its current account deficit declined from an average of EC\$25 million a year in 1990-93 (2.3 percent of GDP) to EC\$5 million in 1994 ( 0.4 percent of GDP), owing mainly to the APUA's stepped-up bill collection efforts. The APUA has had virtually no capital expenditure since 1990 (Statistical Appendix Table 22). The current account has been in deficit since the late 1980s, when desalination and power plant started to operate at a cost that was not covered by water and electricity tariffs. In addition, the APUA's technical and nontechnical losses have remained high 
(20 percent of water and electricity production at end-1994). The authorities have been reluctant to increase tariffs to cover economic costs because of political considerations; electricity tariffs were last raised in 1990, and water tariffs in January 1993. However, the APUA introduced a computerized billing system in 1992 to improve billing and collection procedures. Also, a program for the repair or replacement of water meters has been underway since 1993. On the expenditure side, overstaffing has been a major problem. A management study requested by the authorities in 1993 estimated overstaffing in the water and electricity services at about 40 percent.

The St. John's Development Corporation, which administers the Heritage Quay tourist complex (comprising a hotel, duty-free shops, a casino, and other tourist facilities), registered annual average deficits of EC\$4 million in 1990-94 and has been unable to service its external debt. The Antigua Port Authority has recorded small cash surpluses for several years. However, it has effected only a part of the scheduled transfers that the Government expected to receive for the servicing of the Port's external debt that was subrogated by the Government. Since there is limited scope for increasing docking fees (among the highest in the region), the authorities are working on a program to improve the efficiency of the port including through a voluntary employment reduction scheme. The Central Marketing Corporation is the sole importer of rice, sugar, and five vegetable products not produced locally in sufficient quantity to meet domestic demand (cabbage, carrots, onions, peppers, and tomatoes). The activity of the corporation, which is limited to the marketing of these imports and certain domestic crops, has resulted in near financial balance.

\section{Financial Sector}

\section{Institutional framework}

As a member of the Eastern Caribbean monetary union, Antigua and Barbuda does not have an independent monetary or credit policy. The ECCB issues a common currency, implements monetary policy, and regulates and supervises commercial banking activities in the eight member territories. I In addition, the ECCB is required to keep foreign exchange cover equivalent to at least 60 percent of its demand liabilities, and it is subject to strict limits on credit to member governments. 2/ With these rules, the ECCB generally has maintained noninterventionist monetary policies and acted much like a currency board; its foreign exchange cover was

1/ Other members are Anguilla, Dominica, Grenada, Montserrat, st. Kitts and Nevis, St. Lucia, and St. Vincent and the Grenadines.

2) ECCB holdings of a member government's treasury bills are limited to 10 percent of that government's current revenue, and temporary advances are limited to 5 percent of current revenue. For a description of these limits and a more detailed explanation of the ECCB's institutional arrangements and its responsibilities and powers, see SM/93/241, Appendix IV. 
93 percent of demand liabilities as of December 1994. Net international reserves imputed to a member country are the net foreign asset counterpart of the ECCB's net liabilities to Antigua and Barbuda (currency in circulation less net domestic assets, including the net position with commercial banks).

The financial sector in Antigua and Barbuda includes nine commercial banks, a state development bank, two private finance companies, four credit unions, one leasing company and several insurance companies. 1 / There also is a fledgling offshore financial sector with 37 banks, 15 insurance companies, and 4 international trust companies. Interest rates in Antigua and Barbuda are determined freely except for a minimum of 4 percent on commercial bank savings deposits, established by the ECCB for all its member countries.

\section{Overall developments in the banking system}

Monetization of the Antigua and Barbuda economy (as measured by banking sector liabilities to the private sector relative to nominal GDP) increased during the period 1990-94 as banking services expanded (two commercial banks began operations in Antigua and Barbuda), and domestic interest rates kept pace with international rates.

The banking system's net domestic assets grew much less rapidly than its liabilities to the private sector in 1990-94, partly reflecting a stagnation in credit to the public sector (Table 7). By contrast, in 1994 the growth in net domestic assets was restrained by a slowdown in the growth of credit to the private sector. The net foreign assets of the banking system (including imputed international reserves) rose from EC\$5 million at the end of 1990 to about EC\$140 million at the end of 1994 .

\section{ECCB operations}

\section{a. Operations in Antigua and Barbuda}

Antigua and Barbuda's imputed holdings of net international reserves increased during the period $1990-94$ by EC\$29 million (US\$11 million) to an estimated EC\$106 million (US\$40-million) at end-1994, mainly reflecting the growth in commercial banks' deposits at the ECCB (Table 8). During this period, the ECCB's lending to commercial banks was minimal, and its net credit to the Government rose by only EC\$2 million.

1) The availability of data on the operations of the nonbank financial institutions is limited. 


\section{b. ECCB policy developments}

The ECCB has used monetary policy instruments sparingly, and has kept credit expansion to member governments well within the statutory limits mentioned above. In late 1994 the ECCB implemented the Basle Committee's prudential guidelines on lending practices, which established a statutory limit, equal to 25 percent of the bank's unimpaired capital and reserves, on the credit that a person or group of related persons may obtain from a bank. Previously, the maximum credit amount extended was stipulated in the Banking Act of the individual member country. The ECCB has not yet implemented the Basle Committee's recommendation on requiring that banks maintain an 8 percent risk-weighted capital-asset ratio, but it has sought commercial banks' comments on this recommendation with a view to implementing this capitalasset ratio throughout the region. In 1994 the ECCB also required all banks to adopt practices recommended in the Basle Committee's Statement of Principles on money laundering. Procedures are aimed at proper identification of banks' new customers, cooperation of banks with law enforcement agencies, etc.

The ECCB's efforts to establish a regional home mortgage bank led to an agreement in May 1994 to create the Eastern Caribbean Home Mortgage Bank (ECHMB). Accordingly, the ECHMB will serve as a provider of liquidity to primary lenders in the residential mortgage market. The agreement has been ratified by seven of the elght ECCB member countries, and enabling legislation has been approved in five of them. The ECCB expects the mortgage bank to start operations in the first half of 1995.

\section{Commerctal banks}

Commercial bank liabilities to the private sector grew faster than nominal GDP during the period 1990-94. Domestic interest rates remained roughly in line with international rates except in 1993 when interest rates on savings deposits (fixed by statute at a minimum of 4 percent a year) were higher than international rates. This led to a brisk increase in savings deposits in that year (Table 9).

Commercial bank net domestic assets grew much more slowly than liabil. ities during this period, and their net reserve position (which includes net foreign assets) improved from about minus EC\$10 million at end-1990 to EC\$110 million in 1994; most of the improvement was reflected in an increase in net foreign assets of some EC\$100 million (US\$38 million).

Commercial banks have strengthened their liquidity position in recent years, as the loan-to-deposit ratio decreased from 95 percent in 1990 to 85 percent in 1993 and 83 percent in 1994. In particular, in 1994 the private sector's demand for credit was weak following the March 1994 election, possibly reflecting uncertainty about future policies, particularly in the fiscal area. On the other hand, commercial bank credit to the Government rose substantially in 1993-94; the servicing of most new loans, which carry 
annual interest rates of $11-13$ percent, has been secured through the earmarking of fiscal revenue for debt service (see Appendix III).

Personal loans account for a predominant share of the commercial banks' loan portfolio; this proportion averaged 43 percent of the total in 1990-93, and rose to about 45 percent at the end of 1994 (Statistical Appendix Table 27). The share of credit to the construction sector contracted sharply from 17 percent of total loans at end-1990 to 2 percent at end-1994.

\section{Interest rates}

Domestic interest rates in Antigua and Barbuda, although influenced by domestic liquidity conditions, tend not to deviate substantially from international interest rates. In the period 1990-93, average deposit rates in Antigua and Barbuda fell, albeit with a lag, in response to the decline in international interest rates, and remained approximately unchanged in 1994 despite the rise in U.S. dollar interest rates. Average lending rates also fell during the period 1990-93. As bank liquidity continued to improve in 1994, banks lowered their prime rates even further and reduced their consumer loan rates by about 5 percentage points (Statistical Appendix Table 28). As a result, the spread between average deposit and lending rates fell slightly in both years.

\section{v. External Sector}

\section{Balance of payments}

\section{a. Overall developments}

The current account deficit narrowed from an average of 9.7 percent in 1990-93 to 5.4 percent of GDP in 1994, reflecting a recovery of tourism that outpaced the growth of imports, which were restrained by a decline in domestic investment (Table 10).

The net capital outflow increased from an average of 0.8 percent of GDP in 1990-93 to 2.2 percent of GDP in 1994, owing to a lower level of direct foreign investment that more than offset a decline in scheduled amortization of public sector debt. In addition, in 1994 commercial banks recorded a net capital outflow of US\$10 million, compared with US\$15 million the year before. Antigua and Barbuda's imputed reserves with the ECCB increased by a total of US\$10 million during the period 1990-94.

\section{b. Merchandise trade}

Merchandise exports accounted for less than 8 percent of current account receipts in 1994; one half of this amount comprised re-exports of petroleum products to neighboring islands. The re-export of petroleum derivatives dropped from an average of US\$32 million in 1990-93 to US\$21 
million in 1994, owing to a decline in demand for the blending and storage of naphtha.

Imports provide most of the economy's supply of consumption and investment goods; merchandise imports (including petroleum products for re-export) averaged 92 percent of GDP in 1990-93. This ratio fell to 90 percent in 1994, in part because of the decline in private direct investment. While corroborating trade statistics are not available, the authorities indicated that the share of trade with other members of the OECS has risen thus far during the 1990s.

As mentioned before, Antigua and Barbuda implemented the first stage of CARICOM's CET in January 1995. The changes in the tariff structure, with rates now in the range of $0-35$ percent for nearly all items, entailed a decrease in the average tariff rate. 1/ Soon after implementation of the CET, the Government reduced to zero the rates on a number of items, including milk and poultry meat, because of their importance for household consumption.

\section{c. Tourism}

Antigua and Barbuda is highly dependent on tourism for income and employment; in 1994 the industry generated about 75 percent of current foreign exchange receipts and directly accounted for 12 percent of GDP. After slowing down in the early 1990s, the number of air stayovers picked up In 1993-94 as the number of tourists from Europe increased by an annual average of about 24 percent. In 1994 there was a small increase in cruiseship arrivals to Antigua (2 percent) despite a decrease in cruise-ship visitors to the Caribbean region associated with heightened competition from South American and Pacific destinations (Statistical Appendix Table 34). However, average dafly expenditures by air stayovers decreased in $1993-94$ as a result of competition among hotels (such as all-inclusive tours) and changes in the profile of tourists visiting Antigua and Barbuda toward the younger and less affluent.

The share of European tourists in total arrivals increased from 30 percent in 1990-92 to 38 percent in 1993-94, mainly as a result of a greater number of direct flights (including charters) from Europe and competitively priced tours. The recent changes in the composition of tourists has served to smooth out the seasonal variations in the tourist industry because the

1/ Nonetheless, tariff rates were raised on a number of items under the CET. Many of these rates will be lowered over the period to 1998 in the context of phased reductions in the maximum rates of the CET to 20 percent. In addition, CARICOM is maintaining a rate of 40 percent on some agricultural commodities, which is planned to be reviewed in the light of the results of the Uruguay Round. 
high season for the North American tourist is in the winter, while that of the European is in the summer.

\section{d. $\quad$ ther services and transfers}

Interest obligations on the external public debt fell from an average of 6.4 percent of GDP in the period 1990-93 to 5.5 percent in 1994, partly as a result of lower interest rates on non-U.S. dollar-denominated loans (more than half of Antigua and Barbuda's external debt is denominated in currencies other than the U.S. dollar). In addition, the rescheduling that resulted in two interest-free loans (see below) has contributed to a reduction in the average interest rate from 9 percent in 1990 to 6.8 percent in 1994 (see Table 10). Almost one half of Antigua and Barbuda's external debt bears variable interest rates, at significant spreads over LIBOR.

Net receipts from other nonfactor services decreased from an average of US\$28 million a year in 1990-93 to US\$21 million in 1994 , reflecting an increase in net insurance payments and a recovery of profit remittances abroad by hotels and tour operators. Net official transfers fell from $1990-93$ (US\$2.3 million) to 1994 (US\$1 million) while net private transfers from citizens of Antigua and Barbuda living abroad remained almost unchanged throughout the period.

\section{e. Capital account}

External credit to Antigua and Barbuda was limited in 1990-94 as a result of the country's external arrears problem and weak fiscal performance. Official drawings on loans have been negligible since 1992, resulting in an average of only US\$3.1 million a year in 1990-93. Disbursements of US\$1.6 million in 1994 from the European Development Fund and the Kuwaiti Fund financed road construction. Direct foreign investment declined from US $\$ 30$ million a year in 1990-93 to US\$14 million (about 3 percent of GDP) in 1994 probably because investors were uncertain about future policies--in particular in the tax area--following the March 1994 election. Commercial banks contributed to the deficit in the capital account in 1993-94 when they increased their foreign asset portfolio due to the slackening in the demand for domestic credit.

\section{External debt}

The public sector contracted debt in the early 1980 s mainly in the form of suppliers' credits with official guarantees for the financing of large public investment projects. As the public finances deteriorated, the Government found it more difficult to service its external debt and since the mid-1980s it has been accumulating arrears. Despite a bilateral rescheduling of part of Antigua and Barbuda's external debt in 1990, the stock of external arrears reached US\$337 million (68 percent of GDP) at the end of 1994, of which US\$157 million represented interest arrears, including 
penalty interest (Table 11). Total external debt of the public sector increased from about US\$330 million at end-1990 to US\$400 million in 1994 , equivalent to 81 percent of GDP.

Arrears on debt-service payments for two construction projects--the Royal Antiguan Hotel and a desalination and power plant--accounted for more than one half of the outstanding external arrears as of end-1994. The first credit was from a consortium of banks, denominated in deutsche mark, and guaranteed by the Italian export credit guarantee agency. The second credit was from a Japanese supplier, denominated in U.S. dollars and guaranteed by the Japanese Export-Import Bank. Other arrears are to Brazil, France, other Italian creditors, the United Kingdom, and the United States. Also, there were overdue obligations to the CDB amounting to US\$7.7 million at end-1994 following a partial settlement of arrears in 1994. The remaining arrears preclude new lending by the $\mathrm{CDB}$ to Antigua and Barbuda.

Cash payments on external obligations remained at about US\$9 million on average in 1990-94. Most of the payments were made from public sector revenues earmarked for this purpose, including lease payments for the U.S. military base and the consumption tax on gasoline. These revenues were used to service loans with the U.S. Export-Import Bank and a U.S. supplier, a loan from a U.K. supplier that was restructured into an interest-free loan, and a bank loan that was restructured with a clause providing for a performance-related forgiveness of debt (see Appendix III).

The ratio of contractual debt-service obligations to exports of goods and nonfactor services declined from 12.8 percent in $1990-93$ to 8.1 percent in 1994, largely reflecting a decline in scheduled amortization, as about 85 percent of the debt had fallen into arrears by the end of 1994 . Contractual debt-service obligations with respect to public sector revenue also declined but still remain at over 34 percent.

\section{Exchange and trade system}

\section{a. Exchange rate developments}

Antigua and Barbuda is a member of the Eastern Caribbean monetary union whose currency, the Eastern Caribbean dollar, has been pegged to the U.S. dollar at a rate of EC\$2.7 per U.S. dollar since 1976. Antigua and Barbuda's exchange system is free from restrictions on payments and transfers for all bona fide international transactions.

The real effective exchange rate was approximately constant from 1990 to 1992, but in 1993-94 Antigua and Barbuda's currency appreciated by 5.1 percent in real effective terms, reflecting mainly the strengthening of the U.S. dollar over this period with respect to the currencies of Antigua and Barbuda's trading partners (Chart 1 and Statistical Appendix Table 35). 


\section{b. Trade system}

The trade regime allows the free importation of goods with the exception of certain goods that require a prior license to import. Goods from the OECS member countries and most agricultural imports are duty free, as are imports of capital goods and raw materials for construction under the Fiscal Incentives Act of 1975 and the Hotel Incentives Act. 
ANTIGUA AND BARBUIDA

EXCHANGE RATE DEVELOPMENTS

$(1980=100)$

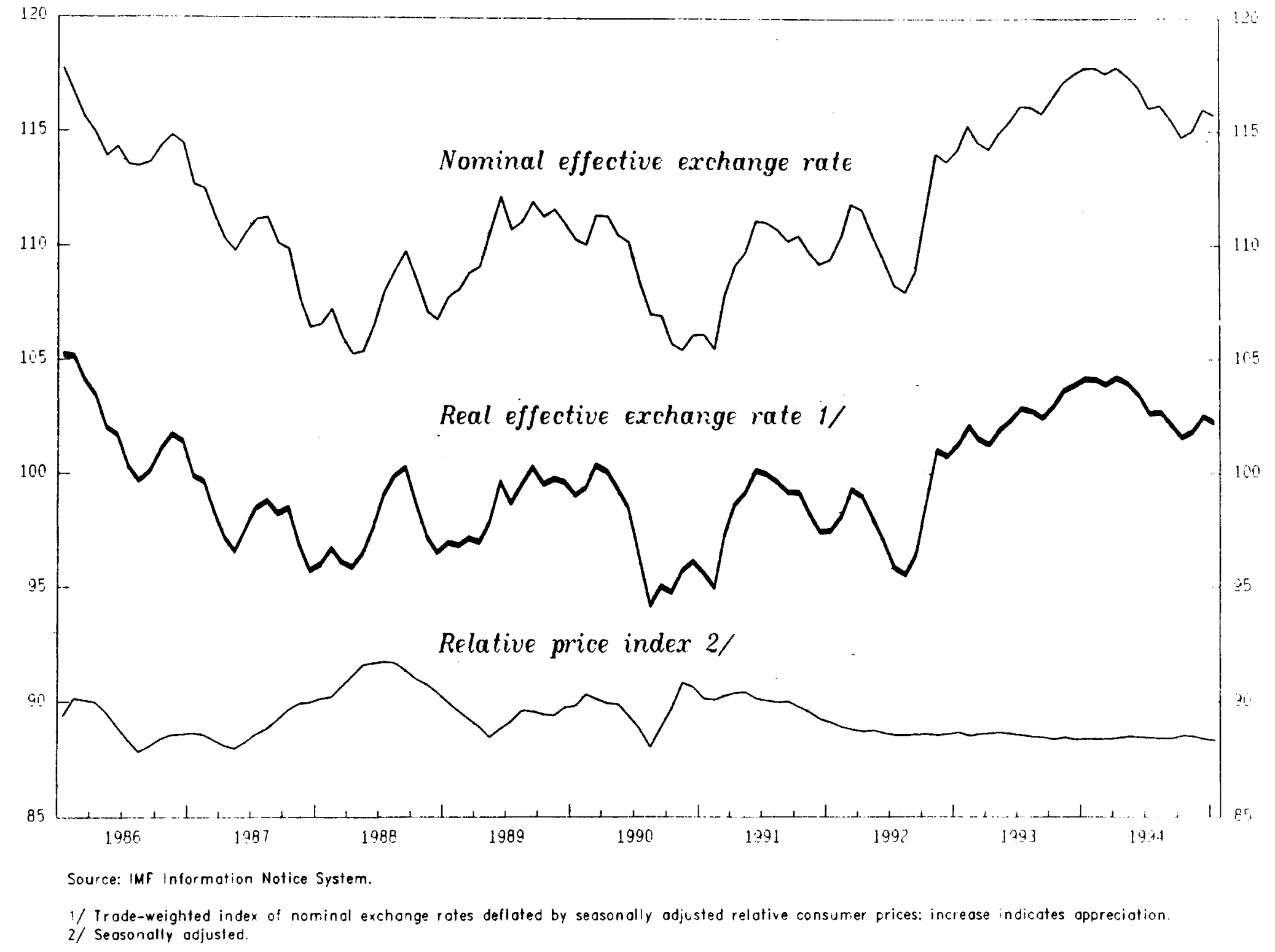


This page intentionally left blank

CInternational Monetary Fund. Not for Redistribution 
Table 1. Antigua and Barbuda: Selected Economic Indicators

\begin{tabular}{|c|c|c|c|c|c|}
\hline & 1990 & 1991 & 1992 & 1993 & $\begin{array}{l}\text { Prel. } \\
1994\end{array}$ \\
\hline \multicolumn{6}{|c|}{ (In millions of Eastern Caribbean dollars) } \\
\hline $\begin{array}{l}\text { Nominal GDP at market prices } \\
\text { Nominal GDP at factor cost } \\
\text { Real GDP at factor cost } \\
\text { (1977 prices) }\end{array}$ & $\begin{array}{r}1,065.6 \\
901.8 \\
362.0\end{array}$ & $\begin{array}{r}1,135.2 \\
969.2 \\
378.1\end{array}$ & $\begin{array}{r}1,174.3 \\
999.4 \\
382.4\end{array}$ & $\begin{array}{l}1,230.0 \\
1,045.7\end{array}$ & $\begin{array}{l}1,331.9 \\
1,123.5\end{array}$ \\
\hline \multicolumn{6}{|c|}{ (Annual percentage change) } \\
\hline $\begin{array}{l}\text { Nominal GDP at market prices } \\
\text { Nominal GDP at factor cost } \\
\text { Real GDP at factor cost } \\
\text { Agriculture } \\
\text { Manufacturing } \\
\text { Construction } \\
\text { Transport and communications } \\
\text { Hotels and restaurants }\end{array}$ & $\begin{array}{r}6.2 \\
6.1 \\
3.5 \\
5.1 \\
3.0 \\
-10.0 \\
4.2 \\
4.6\end{array}$ & $\begin{array}{r}6.5 \\
7.5 \\
4.4 \\
3.0 \\
-5.5 \\
8.4 \\
9.3 \\
-0.1\end{array}$ & $\begin{array}{r}3.4 \\
3.1 \\
1.1 \\
3.8 \\
-6.0 \\
-4.6 \\
3.5 \\
6.1\end{array}$ & $\begin{array}{r}4.7 \\
4.6 \\
3.4 \\
3.3 \\
-5.0 \\
-5.0 \\
6.1 \\
10.0\end{array}$ & $\begin{array}{r}8.3 \\
7.4 \\
4.2 \\
-1.0 \\
2.0 \\
4.0 \\
4.3 \\
6.0\end{array}$ \\
\hline $\begin{array}{l}\text { Consumer prices (average) } \\
\text { Export prices } \\
\text { Import prices } \\
\text { Terms of trade }\end{array}$ & $\begin{array}{r}7.0 \\
4.3 \\
7.1 \\
-2.6\end{array}$ & $\begin{array}{r}5.7 \\
2.7 \\
-1.8 \\
2.9\end{array}$ & $\begin{array}{l}3.0 \\
0.7 \\
0.3 \\
0.4\end{array}$ & $\begin{array}{r}3.1 \\
-1.6 \\
1.6 \\
3.0\end{array}$ & $\begin{array}{r}3.5 \\
-0.3 \\
0.6 \\
-2.3\end{array}$ \\
\hline $\begin{array}{l}\text { Wages (private sector) } \\
\text { Hotels } \\
\text { Nonhotels }\end{array}$ & $\begin{array}{l}7.6 \\
5.8 \\
8.7\end{array}$ & $\begin{array}{l}7.1 \\
3.6 \\
9.3\end{array}$ & $\begin{array}{l}5.7 \\
3.5 \\
7.0\end{array}$ & $\begin{array}{l}6.0 \\
5.1 \\
7.2\end{array}$ & $\begin{array}{l}7.0 \\
6.0 \\
8.0\end{array}$ \\
\hline $\begin{array}{l}\text { Wages (Central Government, } \\
\text { accrual basis) }\end{array}$ & 6.5 & - - & $\cdots$ & 5.0 & 8.0 \\
\hline \multicolumn{6}{|c|}{ (In percent of GDP at market prices) } \\
\hline $\begin{array}{l}\text { Gross fixed capital formation } \\
\text { Gross domestic saving } \\
\text { Public sector } \\
\text { Private sector }\end{array}$ & $\begin{array}{l}27.4 \\
12.6 \\
-2.7 \\
15.4\end{array}$ & $\begin{array}{l}25.2 \\
15.7 \\
-3.4 \\
19.1\end{array}$ & $\begin{array}{l}21.0 \\
13.3 \\
-4.8 \\
18.1\end{array}$ & $\begin{array}{l}24.4 \\
17.6 \\
-3.3 \\
20.8\end{array}$ & $\begin{array}{l}21.7 \\
16.3 \\
-4.0 \\
20.3\end{array}$ \\
\hline
\end{tabular}

Sources: Statistics Division, Ministry of Finance; Labor Commission; OECS; and Fund staff estimates. 
Table 2. Antigua and Barbuda: Summary Operations of the Consolidated Public Sector

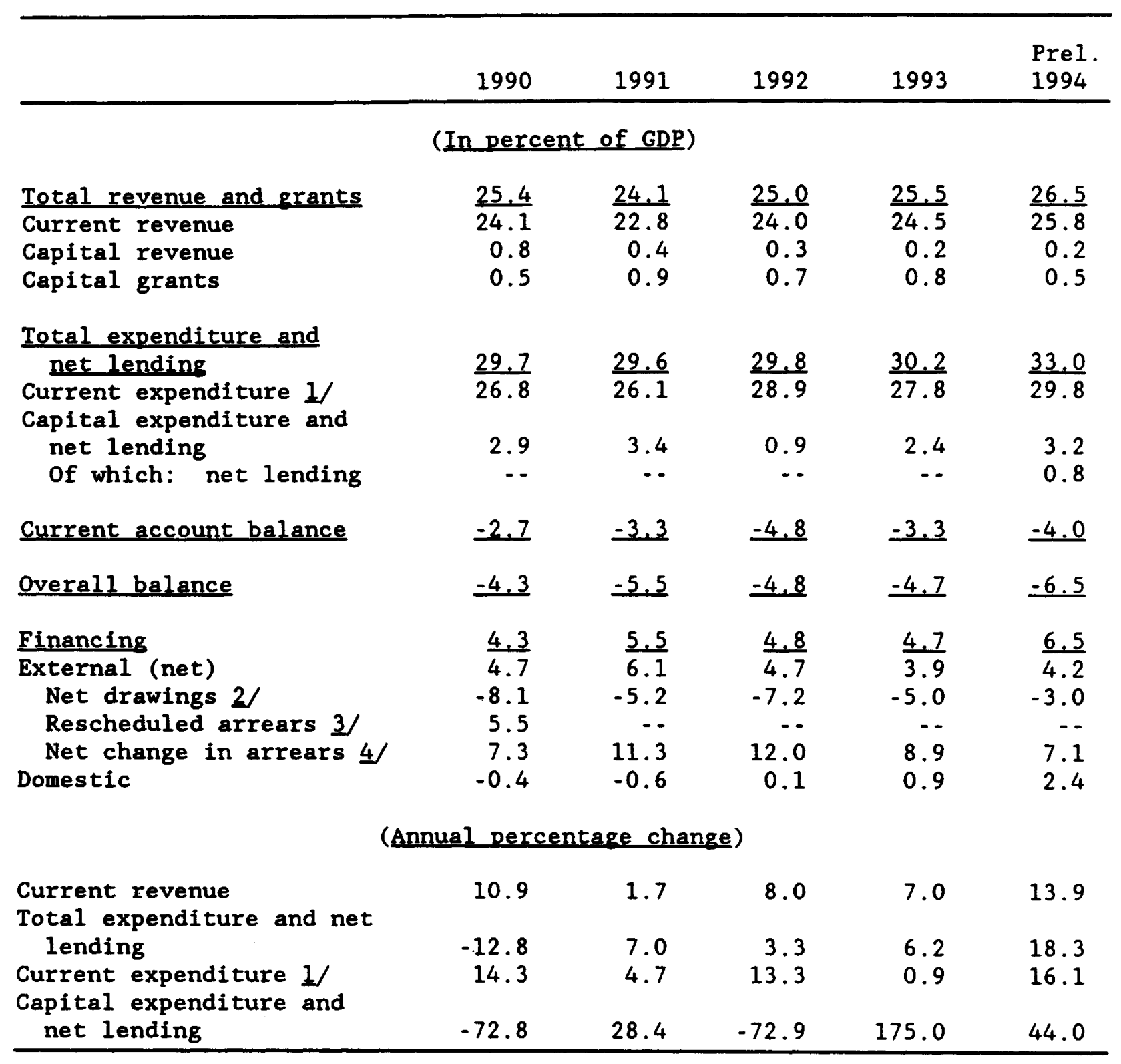

Source: Statistical Appendix Table 22.

1) Includes statistical discrepancy between items above and below the line.

2) Includes change in net foreign assets.

3/ Includes write-off of arrears.

4. Net of rescheduling. 
Table 3. Antigua and Barbuda: Summary Operations of the Central Government

\begin{tabular}{|c|c|c|c|c|c|}
\hline & 1990 & 1991 & 1992 & 1993 & $\begin{array}{l}\text { Prel. } \\
1994\end{array}$ \\
\hline \multicolumn{6}{|c|}{ (In percent of GDP) } \\
\hline Total revenue and grants & 22.1 & 20.0 & $\frac{21.5}{20.1}$ & 20.8 & $\frac{21.4}{10.4}$ \\
\hline Current revenue & $\overline{21.0}$ & $\overline{19.3}$ & $\overline{20.6}$ & 20.1 & $\overline{20.7}$ \\
\hline Capital revenue & 0.7 & 0.4 & 0.3 & 0.2 & 0.2 \\
\hline Capital grants & 0.5 & 0.3 & 0.7 & 0.5 & 0.5 \\
\hline Total expenditure and net lending & 23.9 & $\underline{23.1}$ & 23.7 & 22.9 & $\underline{26.3}$ \\
\hline Current expenditure & $\overline{22.1}$ & $\overline{20.2}$ & $\overline{22.8}$ & $\overline{20.8}$ & $\overline{23.2}$ \\
\hline Of which: wages and salaries & 10.9 & 10.7 & 11.3 & 11.4 & 12.1 \\
\hline goods and services $1 /$ & 5.1 & 3.8 & 5.6 & 3.0 & 5.1 \\
\hline interest & 4.3 & 4.0 & 3.7 & 4.1 & 3.5 \\
\hline Capital expenditure and net lending & 1.8 & 2.9 & 0.9 & 2.1 & 3.2 \\
\hline Current account balance & -1.1 & -0.8 & -2.2 & -0.7 & $\underline{-2.4}$ \\
\hline Overall balance & -1.8 & -3.0 & -2.2 & -2.2 & -4.9 \\
\hline Financins & 1.8 & 3.0 & $\underline{2.0}$ & $\underline{2.2}$ & 4.9 \\
\hline External (net) & $-\overline{0.1}$ & $\overline{1.5}$ & $-\overline{0.2}$ & $-\overline{0.5}$ & $\overline{0.2}$ \\
\hline Net drawings $2 /$ & -1.6 & -- & -2.0 & -2.0 & -1.4 \\
\hline Rescheduled arrears $\underline{3} /$ & 3.5 & -- & -- & -- & -- \\
\hline Net change in arrears 4 / & -2.0 & 1.5 & 1.8 & 1.5 & 1.6 \\
\hline Domestic & 1.9 & 1.6 & 2.2 & 2.6 & 4.7 \\
\hline Change in arrears & 0.7 & 2.0 & 1.3 & 0.2 & 2.6 \\
\hline Other financing & 1.2 & -0.5 & 1.0 & 2.5 & 2.1 \\
\hline \multicolumn{6}{|c|}{ (Annual percentase change) } \\
\hline Current revenue & $\underline{12.1}$ & -0.9 & $\underline{9.0}$ & $\underline{2.2}$ & 11.9 \\
\hline Totel expenditure and net lendins & -15.0 & $\underline{3.6}$ & $\underline{5,3}$ & $\underline{1.4}$ & $\underline{24.4}$ \\
\hline Current expenditure & 1.7 & -1.9 & 15.7 & -4.4 & 20.6 \\
\hline Of which: wages and salaries & 9.9 & 5.9 & 7.6 & 6.2 & 15.0 \\
\hline goods and services 1 / & -21.2 & -21.3 & 54.0 & -43.6 & 82.6 \\
\hline interest & 22.8 & -1.1 & -4.0 & 15.2 & -7.0 \\
\hline Capital expenditure and net lending & -71.6 & 70.5 & -67.7 & 146.0 & 61.0 \\
\hline
\end{tabular}

Source: Statistical Appendix Table 22.

1/ Includes statistical discrepancy between items above and below the line.

2/ Includes change in net foreign assets.

3/ Includes write-off of arrears.

4f Net of rescheduling. 
Table 4. Antigua and Barbuda: Central Government Revenue and Grants (In percent of GDP)

\begin{tabular}{|c|c|c|c|c|c|}
\hline & 1990 & 1991 & 1992 & 1993 & $\begin{array}{l}\text { Prel } \\
1994\end{array}$ \\
\hline Total revenue and grants & $\underline{22.1}$ & 20.0 & 21.5 & 20.8 & 21.4 \\
\hline Current revenue & $\overline{21.0}$ & $\overline{19.3}$ & $\overline{20.6}$ & $\overline{20.1}$ & 20.7 \\
\hline Tax revenue & 18.2 & 16.8 & 17.1 & 17.2 & 17.7 \\
\hline taxes on income & 2.6 & 2.1 & 2.0 & 1.9 & 1.9 \\
\hline consumption duties & 5.4 & 5.6 & 5.7 & 5.8 & 5.6 \\
\hline import duties & 4.1 & 3.7 & 3.6 & 3.6 & 3.5 \\
\hline tourism-related taxes 1 & 3.0 & 2.5 & 2.6 & 2.8 & 2.9 \\
\hline other taxes & 3.2 & 3.0 & 3.1 & 3.1 & 3.8 \\
\hline Nontax revenue & 2.7 & 2.5 & 3.4 & 2.9 & 3.0 \\
\hline $\begin{array}{c}\text { of which: West Indies } 0 \text { il Company } \\
\text { profits }\end{array}$ & $\cdots$ & -- & 0.9 & 0.4 & 0.3 \\
\hline Capital revenue and grants & 1.2 & 0.7 & 1.0 & 0.7 & 0.7 \\
\hline
\end{tabular}

Source: Statistical Appendix Table 24.

1/ Hotel and guest tax, hotel guest levy, travel tax, embarkation tax, cruise-ship passenger tax, and foreign currency levy. 
Table 5. Antigua and Barbuda: Summary Operations of the Rest of the General Government 1/

\begin{tabular}{|c|c|c|c|c|c|}
\hline & 1990 & 1991 & 1992 & 1993 & $\begin{array}{l}\text { Prel. } \\
1994\end{array}$ \\
\hline \multicolumn{6}{|c|}{ (In percent of GDP) } \\
\hline$\frac{\text { Current revenue }}{\text { of which: contributions }}$ & $\frac{4.5}{3.8}$ & $\frac{4.3}{3.7}$ & $\frac{4.9}{4.1}$ & $\frac{5.0}{4.3}$ & $\frac{5.0}{4.2}$ \\
\hline $\begin{array}{l}\text { Current expenditure } \\
\text { of which: benefits }\end{array}$ & $\frac{2.1}{1.0}$ & $\frac{2.3}{0.9}$ & $\frac{2.2}{0.9}$ & $\frac{2.5}{1.0}$ & $\frac{2.8}{1.2}$ \\
\hline Current account balance & $\underline{2.4}$ & $\underline{2.0}$ & $\underline{2.7}$ & $\underline{2.5}$ & $\underline{2.1}$ \\
\hline $\begin{array}{l}\text { Overall balance } \\
\text { Of which: Social Security Scheme } \\
\text { Medical Benefits Scheme }\end{array}$ & $\begin{array}{l}\frac{2.4}{2.0} \\
0.4\end{array}$ & $\begin{array}{l}\frac{2.0}{1.9} \\
0.1\end{array}$ & $\begin{array}{l}\frac{2.7}{2.2} \\
0.5\end{array}$ & $\begin{array}{l}\frac{2.5}{2.1} \\
0.4\end{array}$ & $\begin{array}{l}\frac{2.1}{1.8} \\
0.3\end{array}$ \\
\hline \multicolumn{6}{|c|}{ (Annual percentage change) } \\
\hline $\begin{array}{l}\text { Current revenue } \\
\text { of which: contributions }\end{array}$ & $\frac{12.0}{9.2}$ & $\frac{1.6}{2.6}$ & $\frac{16.7}{14.4}$ & $\frac{8.5}{9.4}$ & $\frac{6.8}{7.3}$ \\
\hline $\begin{array}{l}\text { Current expenditure } \\
\text { of which: benefits }\end{array}$ & $\frac{5.6}{6.3}$ & $\frac{15.8}{-2.9}$ & $\frac{-0.9}{1.9}$ & $\frac{19.8}{24.8}$ & $\frac{23.2}{31.5}$ \\
\hline
\end{tabular}

Source: Statistical Appendix Table 22.

1) Social Security Scheme and Medical Benefits Scheme. 
Table 6. Antigua and Barbuda: Summary Operations of the Consolidated Public Enterprises 1/

\begin{tabular}{|c|c|c|c|c|c|}
\hline & 1990 & 1991 & 1992 & 1993 & $\begin{array}{l}\text { Prel } \\
1994\end{array}$ \\
\hline \multicolumn{6}{|c|}{ (In percent of GDP) } \\
\hline Total revenue and grants & 11.2 & 10.8 & 10.8 & 11.9 & $\underline{12.4}$ \\
\hline Current revenue & $\overline{10.9}$ & 10.3 & 10.8 & 11.7 & 12.4 \\
\hline Capital revenue & 0.3 & $\cdots$ & $\cdots$ & - & - \\
\hline Capital grants & -- & 0.6 & -- & 0.3 & -- \\
\hline Total expenditure & $\underline{16.2}$ & 15.3 & 16.0 & 17.0 & 16.3 \\
\hline Current expenditure & $\overline{14.9}$ & 14.8 & 16.0 & 16.8 & 16.3 \\
\hline Wages and salaries & 2.4 & 2.5 & 2.6 & 2.5 & 2.4 \\
\hline Interest & 5.5 & 4.9 & 5.4 & 4.6 & 4.2 \\
\hline Other 2 & 7.0 & 7.4 & 8.0 & 9.7 & 9.7 \\
\hline Capital expenditure & 1.2 & 0.6 & $-\cdot$ & 0.3 & $\cdots$ \\
\hline Current account balance & $\underline{-4.0}$ & -4.5 & $\underline{-5.3}$ & -5.1 & -3.9 \\
\hline Overall balance & -4.9 & -4.5 & -5.3 & -5.1 & -3.9 \\
\hline \multicolumn{6}{|l|}{ Memorandum items } \\
\hline External financing & 2.8 & 4.6 & 4.9 & 4.3 & 4.0 \\
\hline Net drawings & -6.5 & -5.2 & -5.2 & -3.1 & -1.6 \\
\hline Rescheduled arrears $3 /$ & 2.0 & - & - & - & - \\
\hline Net change in arrears 4 & 7.3 & 9.8 & 10.2 & 7.4 & 5.5 \\
\hline Principal & 5.3 & 5.2 & 5.0 & 3.0 & 1.5 \\
\hline Interest & 4.0 & 4.7 & 5.1 & 4.4 & 4.0 \\
\hline Operating balance & -0.4 & 0.5 & -- & 0.9 & 1.7 \\
\hline \multicolumn{6}{|c|}{ (Annual percentage change) } \\
\hline Current revenue & 9.3 & 1.4 & 7.2 & 13.6 & 14.5 \\
\hline Current expenditure $2 /$ & 36.9 & 6.4 & 11.5 & 9.4 & 9.9 \\
\hline
\end{tabular}

Source: Statistical Appendix Table 22.

1/ The main enterprises are the Antigua Port Authority, Antigua Public Utilities Authority, Deep Bay Development Company, St. John's Development Corporation, and Central Marketing Corporation.

2/ Includes statistical discrepancy between items above and below the line.

3/ Includes write-off of arrears.

4/ Net of rescheduling. 
Table 7. Antigua and Barbuda: Monetary Survey

\begin{tabular}{|c|c|c|c|c|c|}
\hline & \multicolumn{5}{|c|}{ December 31} \\
\hline & 1990 & 1991 & 1992 & 1993 & $\begin{array}{l}\text { Est. } \\
1994\end{array}$ \\
\hline \multicolumn{6}{|c|}{ (In millions of Eastern Caribbean dollars) } \\
\hline Net foreign assets & $\underline{5.7}$ & $\underline{88.4}$ & 90.0 & 117.8 & 138.2 \\
\hline Net (imputed) international reserves & $7 \overline{76.6}$ & $\overline{90.4}$ & $\overline{126.1}$ & $\overline{112.2}$ & 105.8 \\
\hline Commercial bank net foreign assets & -70.9 & -2.0 & -36.1 & 5.5 & 32.4 \\
\hline Net domestic assets & 556.1 & $\underline{557.0}$ & 579.8 & $\underline{629.3}$ & $\underline{676.1}$ \\
\hline Net credit to public sector & 98.3 & 83.8 & 81.9 & 106.4 & 113.8 \\
\hline Central Government & 122.9 & 118.0 & 124.4 & 147.8 & 151.0 \\
\hline ECCB claims & 43.1 & 44.6 & 36.0 & 38.3 & 38.0 \\
\hline Commercial bank claims & 79.8 & 73.4 & 88.4 & 109.4 & 113.0 \\
\hline Statutory bodies & -24.6 & -34.2 & -42.5 & -41.4 & -37.2 \\
\hline Net credit to other financial & & & & & \\
\hline institutions & 1.8 & -3.1 & -35.8 & -30.8 & -28.5 \\
\hline Credit to private sector & 503.1 & 547.4 & 586.7 & 625.3 & 650.3 \\
\hline Other items (net) & -47.1 & -71.2 & -53.0 & -71.5 & -59.5 \\
\hline Monetary liabilities (M2) & 561.8 & 645.4 & 669.8 & 747.1 & $\underline{814.3}$ \\
\hline Money (M1) & $\overline{143.9}$ & $\overline{154.9}$ & $\overline{168.4}$ & $\overline{173.2}$ & $\overline{188.3}$ \\
\hline Currency outside banks & 57.5 & 57.9 & 64.3 & 59.4 & 64.8 \\
\hline Demand deposits & 86.4 & 97.0 & 104.1 & 113.8 & 123.5 \\
\hline Quasi-money & 417.9 & 490.6 & 501.4 & 573.9 & 626.1 \\
\hline Time deposits & 207.6 & 254.6 & 244.2 & 265.9 & 274.4 \\
\hline Savings deposits & 188.1 & 203.6 & 224.1 & 282.9 & 320.0 \\
\hline Foreign currency deposits & 22.2 & 32.4 & 33.1 & 25.1 & 31.7 \\
\hline
\end{tabular}

(Percentage change) 1/

\begin{tabular}{lrrrrr} 
Net foreign assets & $\underline{1.1}$ & $\underline{14.7}$ & $\underline{0.2}$ & $\underline{4.1}$ & $\underline{2.7}$ \\
Net domestic assets & $\underline{1.4}$ & $\underline{0.2}$ & $\underline{3.5}$ & $\underline{7.4}$ & $\underline{6.3}$ \\
\hline Net credit to public sector & -1.4 & -2.6 & -0.3 & 3.7 & 1.0 \\
$\quad \begin{array}{l}\text { Central Government } \\
\text { Statutory bodies }\end{array}$ & 0.2 & -0.9 & 1.0 & 3.5 & 0.4 \\
Net credit to other financial & -1.6 & -1.7 & -1.3 & 0.2 & 0.6 \\
$\quad \begin{array}{l}\text { institutions } \\
\text { Credit to private sector }\end{array}$ & -0.4 & -0.9 & -5.1 & 0.7 & 0.3 \\
Other items (net) & 6.3 & 7.9 & 6.1 & 5.8 & 3.3 \\
Monetary liabilities (M2) & -3.1 & -4.3 & 2.8 & -2.8 & 1.6 \\
Memorandum items & $\underline{2.5}$ & $\underline{14.9}$ & $\underline{3.8}$ & $\underline{11.5}$ & $\underline{9.0}$ \\
Loan-to-deposit ratio & & & & & \\
Velocity & 95.2 & 88.2 & 85.1 & 84.5 & 83.0 \\
& 1.92 & 1.90 & 1.79 & 1.74 & 1.71 \\
\hline
\end{tabular}

Sources: Eastern Caribbean Central Bank; and Fund staff estimates.

1/ Change during period in percent of liabilities to the private sector at the end of preceding period. 
Table 8. Antigua and Barbuda: Eastern Caribbean Central Bank Operations in Antigua and Barbuda

(In millions of Eastern Caribbean dollars)

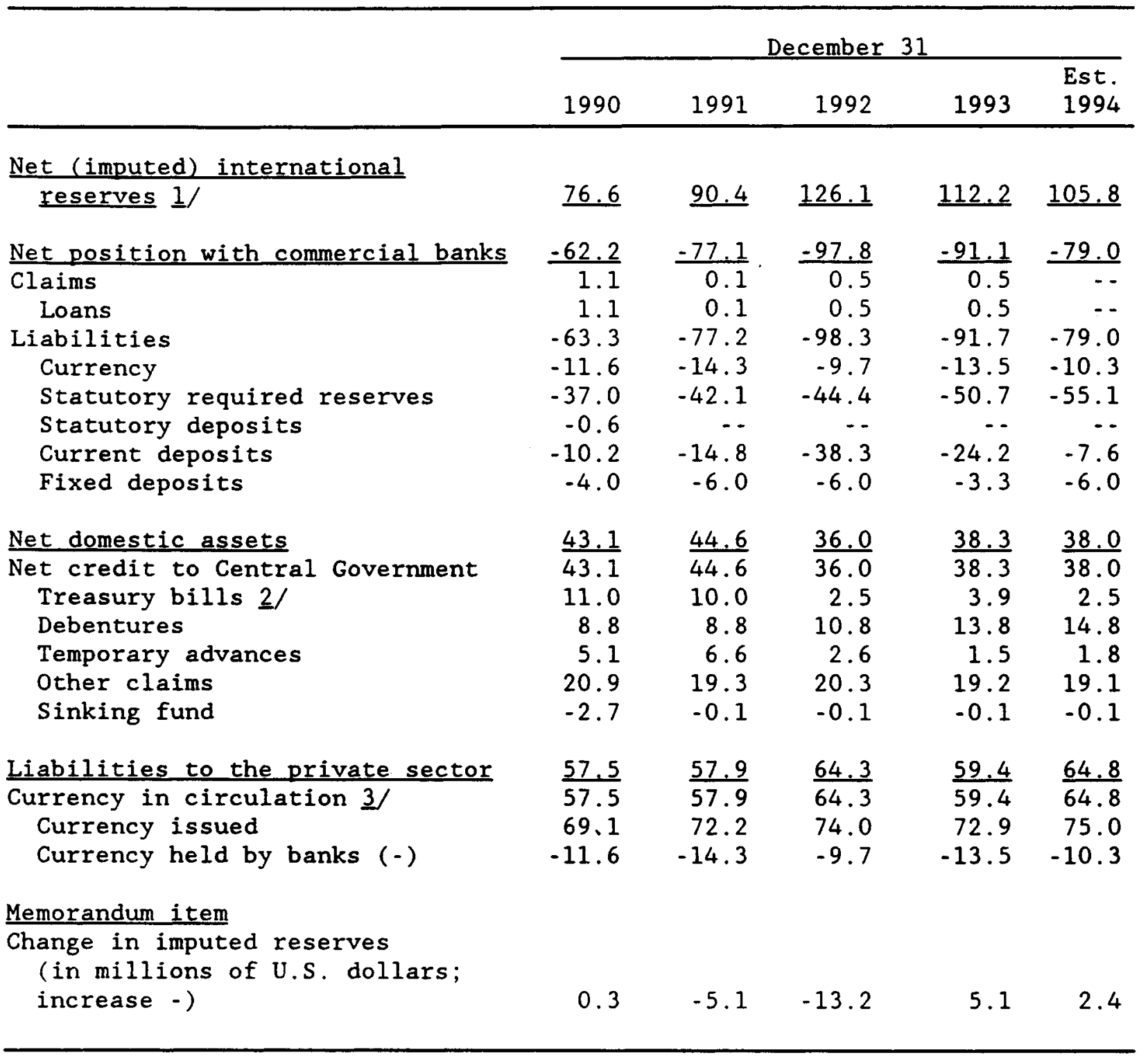

Sources: Eastern Caribbean Central Bank; and commercial banks.

1) Refers to the imputed share of Antigua and Barbuda in the net international reserves of the ECCB; Calculated as the net foreign asset counterpart of net liabilities to Antigua and Barbuda (currency in circueation plus net liabilities to commercial banks less net domestic asseis).

2/ Includes net purchases from commercial banks.

3/ Estimated on the basis of notes issued by the ECCB for Antigua and Barbuda . 
Table 9. Antigua and Barbuda: Summary Accounts of Commercial Banks

\begin{tabular}{|c|c|c|c|c|c|}
\hline & \multicolumn{5}{|c|}{ December 31} \\
\hline & 1990 & 1991 & 1992 & 1993 & $\begin{array}{l}\text { Est. } \\
1994\end{array}$ \\
\hline \multicolumn{6}{|c|}{ (In millions of Eastern Caribbean dollars) } \\
\hline $\begin{array}{l}\text { Net reserve position } \\
\text { Net foreign assets } \\
\text { Net position with ECCB }\end{array}$ & $\begin{array}{r}-\frac{-8.8}{-70.9} \\
62.2\end{array}$ & $\begin{array}{l}\frac{75.1}{-2.0} \\
77.1\end{array}$ & $\begin{array}{r}\frac{61.7}{-36.1} \\
97.8\end{array}$ & $\begin{array}{r}\frac{96.7}{5.5} \\
91.1\end{array}$ & $\begin{array}{r}\frac{111.4}{32.4} \\
79.0\end{array}$ \\
\hline $\begin{array}{l}\text { Net domestic assets } \\
\text { Domestic credit } \\
\text { Public sector (net) } \\
\text { Central Government } \\
\text { Rest of public sector } \\
\text { Other financial institutions (net) } \\
\text { Private sector } \\
\text { Other net assets }\end{array}$ & $\begin{array}{r}513.0 \\
560.1 \\
55.2 \\
79.8 \\
-24.6 \\
1.8 \\
503.1 \\
-47.1\end{array}$ & $\begin{array}{r}512.4 \\
583.6 \\
39.2 \\
73.4 \\
-34.2 \\
-3.1 \\
547.4 \\
-71.2\end{array}$ & $\begin{array}{r}\frac{543.8}{596.8} \\
45.9 \\
88.4 \\
-42.5 \\
-35.8 \\
586.7 \\
-53.0\end{array}$ & $\begin{array}{r}\frac{591.0}{662.5} \\
68.0 \\
109.4 \\
-41.4 \\
-30.8 \\
625.3 \\
-71.5\end{array}$ & $\begin{array}{r}\frac{638.2}{697.6} \\
75.8 \\
113.0 \\
-37.2 \\
-28.5 \\
650.3 \\
-59.5\end{array}$ \\
\hline $\begin{array}{l}\text { Liabilities to private sector } \\
\text { Demand deposits } \\
\text { Savings deposits } \\
\text { Time deposits } \\
\text { Foreign currency deposits }\end{array}$ & $\begin{array}{r}504.3 \\
86.4 \\
188.1 \\
207.6 \\
22.2\end{array}$ & $\begin{array}{r}587.5 \\
97.0 \\
203.6 \\
254.6 \\
32.4\end{array}$ & $\begin{array}{r}\frac{605.5}{104.1} \\
224.1 \\
244.2 \\
33.1\end{array}$ & $\begin{array}{r}\frac{687.7}{113.8} \\
282.9 \\
265.9 \\
25.1\end{array}$ & $\begin{array}{r}\frac{749.6}{123.5} \\
320.0 \\
274.4 \\
31.7\end{array}$ \\
\hline \multicolumn{6}{|c|}{ (Percentage change) 1} \\
\hline Net reserve position & $\underline{3,5}$ & $\underline{16.6}$ & -2.3 & $\underline{5.8}$ & $\underline{2.1}$ \\
\hline $\begin{array}{l}\text { Net domestic assets } \\
\text { Domestic credit } \\
\text { Public sector (net) } \\
\text { Central Government } \\
\text { Rest of public sector } \\
\text { Other financial institutions (net) } \\
\text { Private sector } \\
\text { Other assets (net) }\end{array}$ & $\begin{array}{r}\frac{0.1}{3.5} \\
-3.1 \\
-1.3 \\
-1.8 \\
-0.5 \\
7.1 \\
-3.4\end{array}$ & $\begin{array}{r}-0.1 \\
4.7 \\
-3.2 \\
-1.3 \\
-1.9 \\
-1.0 \\
8.8 \\
-4.8\end{array}$ & $\begin{array}{r}\frac{5.3}{2.2} \\
1.1 \\
2.6 \\
-1.4 \\
-5.6 \\
6.7 \\
3.1\end{array}$ & $\begin{array}{r}\frac{7.8}{10.9} \\
3.7 \\
3.5 \\
0.2 \\
0.8 \\
6.4 \\
-3.1\end{array}$ & $\begin{array}{l}\frac{6.9}{5.1} \\
1.1 \\
0.5 \\
0.6 \\
0.3 \\
3.6 \\
1.8\end{array}$ \\
\hline Liabilities to private sector & $\underline{3.6}$ & $\underline{16.5}$ & $\underline{3.1}$ & $\underline{13.6}$ & $\underline{9.0}$ \\
\hline \multicolumn{6}{|l|}{ Memorandum item } \\
\hline Loan-to-deposit ratio (in percent) & 95.2 & 88.2 & 85.1 & 84.5 & 83.0 \\
\hline
\end{tabular}

Sources: Eastern Caribbean Central Bank; commercial banks; and Fund staff estimates.

1/ Change during year in percent of liabilities to the private sector at end of preceding year. 
Table 10. Antigua and Barbuda: Balance of Payments

\begin{tabular}{|c|c|c|c|c|c|}
\hline - & 1990 & 1991 & 1992 & 1993 & $\begin{array}{l}\text { Prel } \\
1994 \\
\end{array}$ \\
\hline \multicolumn{6}{|c|}{ (In millions of U.S. dollars) } \\
\hline Current balance & -58.2 & -39.7 & $\underline{-33.7}$ & -31.3 & -26.7 \\
\hline Exports, f.o.b. & $\overline{33.4}$ & $\overline{49.5}$ & $\overline{69.6}$ & $\overline{50.9}$ & 40.9 \\
\hline Petroleum products (re-exports) & 19.3 & 32.1 & 45.0 & 30.9 & 21.0 \\
\hline Non-011 exports & 14.1 & 17.4 & 24.6 & 20.0 & 19.9 \\
\hline Imports, c.i.f. $1 /$ & 358.8 & 375.9 & 417.0 & 425.8 & 443.8 \\
\hline Petroleum products & 35.4 & 51.4 & 64.9 & 47.6 & 38.0 \\
\hline Non-oil imports & 323.4 & 324.5 & 352.1 & 378.2 & 405.8 \\
\hline Services (net) & 257.9 & 276.9 & 305.9 & 335.3 & 369.5 \\
\hline Iourist receipts & 298.3 & 308.9 & 331.9 & 376.4 & 415.2 \\
\hline Interest on public debt 1 / & -26.9 & -25.7 & -29.1 & -27.0 & -27.2 \\
\hline Due and paid & -3.0 & -2.9 & -3.0 & -4.0 & -3.2 \\
\hline Due but not paid & -23.9 & -22.8 & -26.1 & -23.0 & -24.0 \\
\hline Other services & -13.5 & -6.4 & 3.1 & -14.1 & -18.5 \\
\hline Transfers & 9.3 & 9.7 & 7.8 & B.3 & 6.8 \\
\hline Private (net) & 7.9 & 6.6 & 4.9 & 5.6 & 5.6 \\
\hline Official (not) & 1.5 & 3.1 & 2.9 & 2.7 & 1.2 \\
\hline Capital account & 7.4 & -3.2 & $-5,1$ & -14.2 & -10.8 \\
\hline Official drawings: & $\overline{3.9}$ & 8.3 & 0.2 & -- & 1.6 \\
\hline Official obligations $1 /$ & -35.7 & -30.5 & -31.7 & -22.9 & -16.3 \\
\hline Ropaymonts made & -8.8 & -5.2 & -5.8 & -5.5 & -5.3 \\
\hline Ropeyments in errears & -26.9 & -25.3 & -25.9 & -17.4 & -11.0 \\
\hline Commercial banks & -2.6 & -25.5 & 12.6 & -15.4 & -10.0 \\
\hline Private direct investment & 41.8 & 44.5 & 13.8 & 24.1 & 13.9 \\
\hline Qverall balance & -50.7 & -42.9 & $-38,7$ & $\underline{-45.5}$ & -37.4 \\
\hline Finaneins & 50.7 & $\underline{42.9}$ & 38.7 & 45.5 & 37.4 \\
\hline Change In foreign assots & -0.3 & $=$ & $=-$ & -- & -- \\
\hline Change in imputed reserves (incroase -) 2 / & 0.3 & -5.1 & -13.2 & 5.1 & 2.4 \\
\hline Accumulation of arrears & 50.7 & 48.0 & 52.0 & 40.4 & 35.1 \\
\hline Reduction of arrears & -21.9 & - & - & -- & $-\quad-$ \\
\hline Rescheduling of arrears and write-offs & 21.9 & $=-$ & -- & $\cdots$ & -- \\
\hline \multicolumn{6}{|c|}{ (In parcent of GDP) } \\
\hline Exports, c.1.f. & 8.5 & 11.7 & 16.0 & 11.2 & 8.3 \\
\hline of which: non-oil & 3.6 & 4.1 & 5.7 & 4.4 & 3.9 \\
\hline $\begin{array}{l}\text { Imports, f.o.b. } \\
\text { of which: non-oil }\end{array}$ & $\begin{array}{l}90.8 \\
81.8\end{array}$ & $\begin{array}{l}88.7 \\
76.6\end{array}$ & $\begin{array}{l}95.9 \\
81.0\end{array}$ & $\begin{array}{l}93.6 \\
83.1\end{array}$ & $\begin{array}{l}90.0 \\
82.3\end{array}$ \\
\hline Tour1st receipts & 75.5 & 72.9 & 76.3 & 82.7 & 84.2 \\
\hline Trade balance & -82.3 & -77.0 & -79.9 & -82.4 & -81.7 \\
\hline Current balance after grants & -14.7 & -9.4 & -7.7 & -6.9 & -5.4 \\
\hline Private direct investment & 10.6 & 10.5 & 3.2 & 5.3 & 2.8 \\
\hline Overall balance & -12.8 & -10.1 & -8.9 & -10.0 & -7.6 \\
\hline
\end{tabular}

Sources: Statistics Division, Ministry of Finance; and Fund staff estimates.

1/ Including orrors and onissions.

2f Change in imputed reserves at the ECCB. 


\section{Zazle 12. Anziglia and Earvuda: Fubitc and Pubicly \\ Guaranteed External Debt}

\begin{tabular}{|c|c|c|c|c|c|}
\hline & 1990 & 1991 & 1992 & 1993 & $\begin{array}{l}\text { Est. } \\
1994\end{array}$ \\
\hline \multicolumn{6}{|c|}{ (In millions of U.S. dollars) } \\
\hline Outstanding debt (end of year) I/ & $\underline{328.6}$ & 351.7 & $\underline{357.6}$ & $\underline{362.2}$ & $\underline{401.3}$ \\
\hline Valuation adjustment $2 /$ & $\underline{12.0}$ & -1.9 & -13.7 & -12.1 & 19.6 \\
\hline of which: on stock of arrears & $\overline{12.4}$ & 1.1 & $\overline{-11.5}$ & $\overline{-10.6}$ & $\overline{19.0}$ \\
\hline Drawings on official debt & $\underline{3.9}$ & $\underline{8.3}$ & $\underline{0.2}$ & $\underline{-}$ & $\underline{1.6}$ \\
\hline Scheduled debt obligations & $\underline{62.5}$ & $\underline{56.3}$ & $\underline{60.9}$ & 49.9 & 43.5 \\
\hline Interest & $\overline{26.9}$ & $\overline{25.7}$ & $\overline{29.1}$ & $\overline{27.0}$ & $\overline{27.2}$ \\
\hline Principal & 35.7 & 30.6 & 31.8 & 23.0 & 16.3 \\
\hline Cash payments on debt obligations & $\underline{11.0}$ & 8.1 & $\underline{8.8}$ & 9.4 & $\underline{8.5}$ \\
\hline Interest & 3.0 & $\overline{2.9}$ & $\overline{3.0}$ & $\overline{4.0}$ & $\overline{3.2}$ \\
\hline Principal & 8.8 & 5.2 & 5.8 & 5.4 & 5.3 \\
\hline Change in arrears (net) & $\underline{28.8}$ & $\underline{48.0}$ & $\underline{52.0}$ & 40.3 & 35.0 \\
\hline Interest & $\overline{15.0}$ & $\overline{22.8}$ & $\overline{26.1}$ & $\overline{22.5}$ & $\overline{24.0}$ \\
\hline Principal & 14.1 & 25.3 & 25.9 & 17.4 & 11.0 \\
\hline \multicolumn{6}{|l|}{ Rescheduling and write-offs } \\
\hline of arrears & $\underline{21.9}$ & - & ․ & - & ․ \\
\hline Interest & 9.1 & -- & $\cdots$ & $\overline{-}$ & $\cdots$ \\
\hline Principal & 12.8 & $-\cdot$ & $-\cdot$ & -- & - \\
\hline Stock of arrears (end of year) & 163.5 & 212.7 & 253.3 & 283.1 & 337.1 \\
\hline Interest & 61.5 & 85.0 & $\overline{106.6}$ & $\overline{125.1}$ & $\overline{157.2}$ \\
\hline Principal & 102.0 & 127.7 & 146.7 & 158.0 & 179.9 \\
\hline \multicolumn{6}{|c|}{ (In percent) } \\
\hline $\begin{array}{l}\text { Scheduled debt service/exports } \\
\text { of goods and nonfactor services } \\
\text { Scheduled debt service/total }\end{array}$ & 15.6 & 12.9 & 12.7 & 9.8 & 8.1 \\
\hline public sector revenue & 62.4 & 54.0 & 55.8 & 45.2 & 34.0 \\
\hline Debt outstanding/GDP & 83.2 & 83.6 & 82.2 & 79.2 & 81.3 \\
\hline Stock of arrears/GDP & 41.4 & 50.6 & 58.2 & 61.9 & 68.3 \\
\hline Average interest rate & 8.6 & 7.5 & 8.2 & 7.5 & 7.1 \\
\hline
\end{tabular}

Sources: Ministry of Finance; Eastern Caribbean Central Bank; and Fund staff estimates.

1/ Including penalty interest.

2) Including exchange rate valuations.

CInternational Monetary Fund. Not for Redistribution 


\section{Summary of Fiscal Measures}

Changes in 1991

In February 1992 the cruise-ship passenger tax was increased from US\$2 to US\$3 per passenger.

Changes in 1992

In March 1992 the departure (embarkation) tax was increased to EC\$25 for foreigners and EC\$20 for nationals.

Changes in June 1994

- The restaurant sales tax of 7 percent, previously applicable to restaurants in hotels, was extended to nonhotel restaurants.

- The customs service tax was raised from 2.5 percent to 5 percent of the c.i.f. value of imports.

- The cruise-ship passenger tax was raised from US\$3 to US\$6 per passenger.

Changes in December 1994/January 1995

- The hotel guest tax was raised from 5 percent to 6.5 percent of the hotel bill.

- An education levy on wages and salaries was introduced at the rate of 2.5 percent on annual earnings of EC $\$ 6,500-60,000$, and a marginal rate of 5 percent on annual earnings in excess of EC $\$ 60,000$. Income from selfemployment also is subject to this tax.

- The departure (embarkation) tax was increased by EC\$5, to EC\$30 for foreigners and EC\$25 for nationals.

- The rate of contribution for medical benefits was raised from 5 percent to 7 percent of wages and salaries, and the ceiling for chargeable earnings was raised from EC $\$ 2,500$ a month to EC $\$ 4,500$ a month.

- The Business Licence Act was introduced, expanding the registration of businesses and replacing certain trade and professional licenses. The annual registration fee for citizens varies from EC $\$ 50$ to EC $\$ 5,000$, depending on the nature of the business (e.g., EC\$1,000 for professional services). Fees for noncitizens range from EC $\$ 50$ to EC $\$ 10,000$.

- Property tax valuations were raised for the first time in several years in seven of ten sections of St. John's, including all commercial establishments; the estimated three- to four-fold increase in valuation combined with rate reductions is expected to yield approximately a 100 percent increase in property taxes on these properties. 
- The business tax was extended to self-employed persons.

- The Customs Duties Act of 1993 became effective, implementing the first stage of the CARICOM common external tariff, the net effect of which was to lower the average import tariff.

- Consumption tax rates were raised to compensate for the revenue effect of the decline in the average import tariff just mentioned. The harmonized nomenclature utilized by CARICOM countries in the import tariff was adopted, with all rates on an ad valorem basis except for those on petroleum products.

- The Revenue (Miscellaneous Provisions) Act of 1994, in addition to increasing the departure tax, hotel guest tax, and customs service tax mentioned previously, increased several licenses, fees and duties, including the following:

\section{(Amounts in Eastern Caribbean dollars)}

Annual liquor licenses 1

Retail liquor sales

Retail beer sales

Hotel liquor license

Importer's license

Annual tobacco license

Annual motor vehicle license $2 /$

Private motor cars

Motorcycles

Commercial vehicles

Driver's licenses $3 /$

One-year

Three-year

Transfer tex in form of stamp

duty paid by seller 4/

$$
\begin{array}{r}
180 \text { to } 1,000 \\
100 \text { to } 500 \\
2,400 \text { to } 9,600 \\
2,000 \\
10 \text { to } 500
\end{array}
$$

125 to 300

25 to 100

150 to 1,500

$$
\begin{aligned}
& 20 \text { to } 40 \\
& 50 \text { to } 100
\end{aligned}
$$

5 percent

$$
\begin{array}{r}
400 \text { to } 1,200 \\
200 \text { to } 800 \\
3,000 \text { to } 12,000 \\
3,000 \\
50 \text { to } 1,500
\end{array}
$$

125 to 450

25 to 450

250 to 2,500

50 to 75

120 to 200

7.5 percent

1 Varies by location.

2) Varies by type and engine size.

3 Varies by type of vehicle.

4. In addition to 2.5 percent tax paid by purchaser. 
1. Texes on income, profits and payroll

Taxes on income

1.1 Law Cap. 266 Amended 1980
Basis of assessment. The basis of assessment is the income of the previous calendar yoar for nonresidents and the accounting year for companies. Hage and salary earners are not subject to income tax.

Liability to income tex Nonresident individuals are taxed on income from any source within Antigua. Income tax 1s levied on the income of any company registered in Antigue and on the income of foreign companies which is generated in Antigua.

Assessable income is defined as (a) earnings in money or kind from employnent;

(b) profits from trade;

(c) dividends, interest, and dis counts:

(d) pensions and annuities:

(e) property income;
Exemptions

Official woluments of Governor General, incore (other than that derived from trade) of local authorities, statutory bodies, friendly societies, ecclesiastical, charitable, and educational institutions. Also, emoluments payable to permanent consular members, armed forces, wound and disability pensions and sratuities paid to armed forces. and sratuities paid to armed forces, income of the Government Development. Bank and Government Savings Bank, and public officers, allowances to the Chief Justice of the West Indies and scholarships and other educational allowances. Income derived from investments on Government's treasury bills and development bonds.

Deductions include normal operatins costs, interest, rent of land and industrial building depreciation, previous losses against income, income coming under th Hotels Aid Ordinance Act of 1952, the Fiscal Incentives Ordinance 1976 Contributions to charitable, religious educational, and scientific institutions. Dividends are taxable in the shareholder's hands.

\section{Personal reliefs}

Income tax is payable at graduated rates on chargeable income of nonresident individuals and at at flate for compantes as follows:

\section{(a) Individuals}

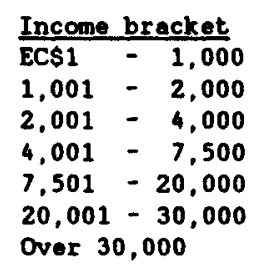

$\frac{\text { Rate of tax }}{\text { (percent) }}$
From $21 / 2$ to 5
From $71 / 2$ to 10
From 10 to 20
From 20 to 30
From 35 to 45
From 50 to 55
55

(b) Companies 
(f) undistributed profits of . company controlled by no more than five persons may be decened income of the shareholders and taxed as such, provided that it could be distributed without detriment to the company's existins business. Subsequent distribution of such income 18 excluded from tax.

1.2 Business Tax Act Law $22 / 82$, enended in 1994

1.3 Nonresident withholding on account

1.4 Education levy Board of Education Act. 1994

\section{Iaxes on property}

2.1 Land and bulldings Laws: Cap. 267 Law $13,15,17$ Amended 1979

2.2 Undeveloped land tax omed by nonbelongers
$A$ tax on declared undeveloped land.
Individuals deriving income from a business are 1iable for the payment of a tax on that income.

Payments made to nonresident in None.

the form of a dividend, interest on discounts, rental, lease, premium on income, royelties.

management fees, comission,

annuities, or any other payment

an of income neture ore pubjent

to withholding on account.

A levy on wages and salaries

(1ncludins overtime, but

excluding tips and other nomwage

income is withheld by the

employer and paid into an

Education Fund on weekly or

monthly basis. Income from self-

employment also is subject to

this levy: self-employed persons

pay the levy monthly. A Board of

Education established by the

Government will authorizo

expenditures for education from

this Fund.

Basis of assessment. Annual rental value of house or area of land, or any property. Subject

Government buildings used for public purposes, buildings owned and used by the University of the West Indies,

to revision every five years.

butldings used for religious purposes.

None
25 percent of chargeable income.

$\begin{array}{ll}\text { Individual } & -20 \text { percent } \\ \text { Companies } & -40 \text { percent } \\ \text { Any other income } & -25 \text { percent }\end{array}$

The levy is payable on wages and salaries, including overtime, and on self-employment income above EC\$6,500 a year, as follows:

\section{Annual Incone}

Rate of tax

Ec\$6, 500-60,000 Over EC $\$ 60,000$

and and buildings, from 3.5 percent to 20 percent of assessed annual rental value; for land, less than one half acre exempt.

5 percent first year, increasing 5 percent each additional year to a maximum of 20 percent. 
Ant1gua and Barbuda: Tax Surmary (Continued)

(As of January 31, 1995)

\section{$\operatorname{Tax}$}

2.3 Property transfer tax on nonbelongers

2.4 Property transfer tax (part of stamp duty ordinance)

\section{Taxes on soods and services}

3.1 Consumption Tax Act $28 / 93$

3.2 Consumption tax on fuel

3.3 Excise duty

3.4 Hotel accommodation tax - Lan 14/62

3.5 Hotel guest taxLaw $14 / 62$

3.6 Hotel guest levy $19 / 82$

3.7 Embarkation tax

3.8 Travel tax - Law $4 / 78$

3.9 Telecomunications tax - Law $7 / 74$

3.10 Cruise passenger tax

Nature of Tax

Exemptions and Deductions

None.

A tax on transfers by non-

belongers to be paid by the

vendors.

A tax on property purchased by a None

noncitizen to be paid by the

purchaser.

Paid by means of stamps:

None.

Consumption taxes comprise ad valorem taxes on goods (mainly consumer durables and. luxuries) payable by the importer. The same rate is applicable to locally produced goods (0.8.) rum, cigarettes, and furniture) For purposes of the tax, imports For purposes of the tax,
are valued on the basis of c.i.f. value plus impart duty.

Specific.

Levied on locally manufactured rum.

Paid by hotel proprietor.

Payable by guest on hotel bill. including room, food, and drink charges.

Payable on each hotel bill.

Passengers embarking at an airport for external flights.

Payable on tickets abroad.

International telephone calls

Visiting cruise-ship passengers.
The Act incorporates a schedule of 12 exemptions, including military arms and munitions for government use; re-imports on which tax has been paid; articles for diplomatic personnel; certain personal effects; articles for use in and construction of schools; boats for shipping or commercial fishing and items pertaining to them; and second-hand clothing imported for charity.

None.

Collected only when excise duty is more than consumption tax.

None.

None.

None.

Diplomats, in transit passengers

Antiguans under 16 years and diplomats. people travelling to seek medical treatment.

None

None
Rates

5 percent of the capital gain.

5 percent of the value of property purchased.

2.5 percent paid by purchaser, 7.5 percent paid by vendor.

Most rates on taxable items are 15 percent, 20 percent or 30 percent. A few items (e.g., some motor vehicles firearms) are taxed at 50 percent.

EC\$2.95 per gallon of motor spirits. EC\$2.89 per gallon of gas oil, diesel oll.

EC\$0.93 per gallon of kerosene.

EC\$1.32 per gallon.

Hotels: from EC\$2.50 per person per night to EC\$10.

6.5 percent.

2 percent.

Embarkation: EC\$30 for foreigners and EC\$25 for nationals.

10 percent of cost of ticket.

20 percent of cost of telephone bill.

US\$6 per passenger 


\section{Tax}

Exemptions and Deductions

Mone.

None.

Levied on insurance policies other than vehicle insurance.

Tax paid by casino operators.

None.

Levied on betting and lottery None. tickets.

Levied on the sale or resale of Hone.

time-sherins intervals.

Levied on the sale or resale of time-sharing intervals.

Mone.

3.16 Ifme-sharing service $\operatorname{tax}$

4. Taxes on international trade

4.1 Import tariffs - Law 27/93

4.2 Import surcharge

4.3 Customs service charge Law 6/86, amended by Law $18 / 94$
The tariff structure implements the first stase of the CARICOM common oxternal tariff (CET), and is based on the harmonized system nomenclature of CARICOM.

Exceptions to standerd CARICOM rates are specified in List A (rates on items not needing protectlon; thay may be lowered

at the national government's discretion): List B (national list of exceptions generally lower than the CET; may be lower than the CET; may be
changed only by decision of CARICOM); and List $C$ (rates may be raised for revenue purposes at the discretion of the national government).

Levied on c.i.f. value of goods not subject to consumption tax.

Levied on the c.i.f. value of all imports.
Imports exempt under industrial incentives legislation, or exempt under Hotel Alds Oxdinance; Imports by diplomatic personnel. Imports from within CARICOM are exempt from duty $1 f$ thay satisfy requirements on orisin of production.

None.

Imports exempt under industrial

incentives legislation and under the Hotel Aids Ordinance. Diplomatic personnel.
Rates

10 percent of cost of ticket.

3 percent of value of premium.

Rate varies according to casino size; EC\$300,000 maximum rate.

15 percent.

5 percent of the purchase price of the time-sharing interval, paid by the developing owner.

5 percent of the purchase price of the time-sharing interval, paid by the managing asent.

Rates are in the range of 0-40 percent for nearly all items, and 70 percent for munitions. 
Nature of Tax
$\operatorname{Tax}$
Exemptions and Deductions

None.

Transactions w1th value of EC\$25 or 1ess.

exchange by comercial bank payable by the customers.

5. Other taxes

5.1 Stamp duties Lam Cap. 270 amended 1977 and 1994

5.2 Stamp Duty (Amendment) Act 1986

\section{Licenses}

6.1 Bank licenses

\subsection{Licenses under the} International Business Corporation Act 28/82

6.3 Business License $17 / 94$

\subsection{Licenses payable by} insurers $21 / 82$
Ad valorem and specific duties on a range of specified instruments including receipts, cortificates. and other legel documents.

\section{A tax payable by any bank or financial institution which} provides loans to noncitizens.

\section{Annual license foe for domestic} commercial banks.

\section{Annual license payable by}

businesses registered under the

Act.

Annual license payable by persons practicing a profession for profit.
Agreements of which the subject matter has a value of EC\$25 or less; agreements for the hire of a laborer or anufacturer: and sales agreements.

Antigua and Barbuda Development Bank.

Mone.

None.

None.

None.
Lobsters EC\$O.50 per pound.

Fish EC\$O. 10 per pound.

1 percent.

\begin{tabular}{cc} 
Subject matter value & Stamp duty \\
\hline (1n Eastern Caribbean dollars) \\
$25-250$ & 10 \\
$251-500$ & 20 \\
$501-750$ & 40 \\
$751-1000$ & 60
\end{tabular}

(duty rises by EC\$20 per $\$ 250$ increment in the value of the subject matter).

1 percent of the face value of the loan.

Antigua Commercial Bank EC $\$ 10,000$, other commercial banks EC\$20,000.

Banks : US $\$ 5,000$

Trust companies: US\$2,500.

Insurance companies: US\$2,500.

Others: US\$500.

Annual fees for citizens vary from EC\$50 to EC\$5,000 depending on nature of

business (EC $\$ 1.000$ for professional services). Fees for noncitizens range from EC\$50 to EC $\$ 10,000$.

For insurance companies: EC\$5,000.

For insurance agents: EC\$500.

For insurance brokers: EC\$500.

For special insurance brokers: EC $\$ 1,000$ For insurance salesmen: EC\$50.

\section{EC\$2,000 year.}

Licenses range from EC\$400 to EC\$1,200 a year depending on the location of the establishment (EC\$200 to EC\$800 to sell only beer). 
6.7 Hotel license

6.8 Importers 11 cense

6.9 Iavern. club license

6.10 Tobacco 11 conse

6.11 Motor vehicle 11 cense

7. Eees

7.1 Passport rees

7.2 Citizonship

7.3 Extension of Imaleration permit

7.4 Driver's licenses

7.5 Other fees

7.6 Navigation services
Hature of $\operatorname{Tax}$

License to sell Liquor, based on number of roons.

License to import 11quor.

License to seill liquor.

Annual 11conse to sell tobecco products.

Annual 11cense. Rate depends on engine size, and for comerelel vehicles on the type of vehicle.
Mone.

None.

Exemptions and Deductions

None.

None.

Mone

Exaptions and Deduction.

Rates

From EC $\$ 3,000$ to EC\$12,000 a year depending on number of hotel rooms.

EC\$3,000 year.

EC $\$ 400$ to EC\$2.000 year, depending on location.

eC\$50 for retall, EC\$1,000 for wholesale, EC\$1,500 to Import and distribute.

Motorcycles, EC\$100 to EC\$250; private sutomobiles, EC\$125 to EC\$450; and comerercial vehleles. EC\$250 to EC\$2,500.

Ec\$20.

Mone.

None.

Mone.

None.

Licenses for 1 and 3 years.

Various.

A charge per landing for navigation services provided.
None. cesso.

CARICOY nembers EC\$300. Nonmembers EC\$1, 000 .

CARICOA nembers ECS5O. Nommembers EC\$300.

Motorcycle ECS50 ( 1 year) and EC\$120 (3 years): motor vehicles EC\$60 (1 year). and EC\$150 (3 years); chauffeur EC\$75 (1 yoar) and EC\$200 (3 years).

Driver exanination ECS30; motor vehicle ragistration ECS100; visitors' permit to drive EC\$50: registration of change of ownership EC\$50; duplicate license EC\$40; examination of vehtcles EC\$30.

For aircraft up to 12,500 lbs: EC\$15

From 12,501 to 75,000 1bs: EC\$30 From 75,001 lbs: EC\$90 


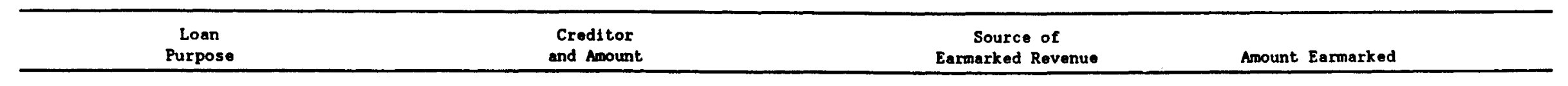

\section{External Loans}

1. Loans contracted for st. John's Harbour improvement (including dredging).

2. Rescheduling of obligations related to construction of Halcyon Cove Resort.

3. Rescheduling of obligations related to construction of a desalination and power plant.

4. Loans contracted to support the operations of the state utilities company (APUA) and the Antigua Port Authority.

\section{Domestic Loans}

1. Loan for current expenditure.

2. Loan to pay salary arrears (EC\$2.2 million) and to consolidate previous loans (EC\$2.8 million).

3. Credit onlent to airline (LIAT) to ready it for privatization.

4. Loan for roads project: disbursement of EC\$1.9 million in July 1994 was consolidated with previous loan of EC\$2.2 million.
Antigua Masonry Products (supplier. subsidiary of DEVCON--U.S. company) Loan amount was US\$27.6 million.

Credit Suisse Bank. In 1990 an agreement was reached to repay outstanding arrears (including interest arrears) of Sw F 20.7 million in 1990-95. The loan is interest free; half of the outstanding obligations are written off provided that the new amortization schedule is observed. Otherwise, the loan would revert to the original terms, including an ennual interest rate of 8 percent.

Foster Wheeler Power Products (British supplier). In 1990 on egreement was reached to repay outstanding obligations (including arrears) of us\$22 million in 1990-95. The loan is interest free.

U.S. Export-Import Bank. Loan amount was US\$7. 1 million.

Swiss-American Bank. Amount of 10-year loan was EC\$2 million, disbursed in April 1994.

Swiss-American Bank. Amount of 5-year loan was EC\$5 million, disbursed in December 1993.

Finance and Development Co. Ltd. Amount of 10-year loan was EC\$10 million.

Finance and Development Co. Ltd. Amount of 5-year loan was EC\$4.15 million. a) As of 1987 proceeds from lease of U.S. Dilitary base.

b) As of April 1991 operating revenue of the Antigua Port Authority (collected by New Port Antigua Ltd.).

As of 1990 proceeds from the consumption tax on gasoline collected by the West Indies oil Company are deposited in an account at Swiss-American Bank (SAB) in Antigua. Final payment is made from the SAB account.

As of 1990 proceeds from the consumption tax on gasoline are deposited in an account at $S A B$ in Antigua by the West Indies Oil Company. Final payment is made from the SAB account.

As of 1987 proceeds from lease of U.S. military base.

Consumption tax on petroleum products.

Consumption tax on petroleum products.

\section{Consumption tax on petroleum products.}

Consumption tax on petroleum products.
EC\$113,766 a month

EC\$146,410 a month.

a) US\$500,000 quarter.

b) Variable

Sw F 1.2 million a year in semiannual installments.

US\$4 million a year in semiannual installments.

US\$125,000 quarter

EC\$28.694 a month.

month

EC $\$ 98,010$ a month. 
Antigua and Barbuda: Earmarking of Government Revenue to Service Selected Loans (Concluded)

\begin{tabular}{|c|c|c|c|}
\hline $\begin{array}{c}\text { Loan } \\
\text { Purpose }\end{array}$ & $\begin{array}{l}\text { Creditor } \\
\text { and Amount }\end{array}$ & $\begin{array}{c}\text { Source of } \\
\text { Earmarked Revenue }\end{array}$ & Amount Earmarked \\
\hline 5. Loan for roads project. & $\begin{array}{l}\text { Finance and Development Co. Ltd. Amount } \\
\text { of 2-year loan was ECS4.5 million, dis- } \\
\text { bursed in July } 1994 \text {. }\end{array}$ & Consumption tax on petroloum products. & Not available. \\
\hline $\begin{array}{l}\text { 6. Loan in January } 1995 \text { to } \\
\text { finance current expenditure } \\
\text { (backpay, regional organizations, } \\
\text { etc.). }\end{array}$ & $\begin{array}{l}\text { Bank of Antigus. Amount of } 15 \text {-year loan } \\
\text { was ECS20 million. }\end{array}$ & Consumption tax on petroleum products. & EC $\$ 257.610$ a month. \\
\hline $\begin{array}{l}\text { 7. Loan to refurbish } \\
\text { airport (EC\$10 million) } \\
\text { and for backpay of hospital } \\
\text { speciallsts (ECS1 million). }\end{array}$ & $\begin{array}{l}\text { Bank of Antigua. Amount of } 15-y e a r \text { loan } \\
\text { is EC\$1 million, of which about EC } \$ 6 \text { mil- } \\
\text { lion was disbursed in 1993, and EC\$5 } \\
\text { million in } 1994 \text {. }\end{array}$ & Alrport landing fees. & EC\$159,200 a month. \\
\hline $\begin{array}{l}\text { 8. Loan to settle arrears on } \\
\text { airport project. }\end{array}$ & $\begin{array}{l}\text { Caribbean Banking Corp. Amount of } \\
4.5 \text {-year loan was EC\$6.75 million. }\end{array}$ & $\begin{array}{l}\text { Government's share of West Indies Oil } \\
\text { Co. proffts. }\end{array}$ & $\begin{array}{l}\text { Payments of EC } \$ 378,000 \text { a } \\
\text { quarter. }\end{array}$ \\
\hline $\begin{array}{l}\text { 9. Loan for purchase of embassy } \\
\text { property in Washington. }\end{array}$ & $\begin{array}{l}\text { Antigua Commercial Bank. Amount of } \\
15 \text {-year loan was EC } \$ 1.7 \text { million. }\end{array}$ & $\begin{array}{l}\text { Government receipts from offshore } \\
\text { companies. }\end{array}$ & EC\$18,268 anth. \\
\hline 10. Loan to purchase textbooks. & $\begin{array}{l}\text { Antigua Commercial Bank. Anount of } \\
\text { 1-year variable loan was EC\$2.5 million, } \\
\text { disbursed in } 1994 \text {. }\end{array}$ & Education levy. & Not available. \\
\hline $\begin{array}{l}\text { 11. Loan to consolidate arrears, } \\
\text { previous loans, purchase of } \\
\text { CAT-Scan (EC\$2.7 million), and } \\
\text { other equipment. }\end{array}$ & $\begin{array}{l}\text { Antigua Commercial Bank. Amount of } \\
15 \text {-year loan was EC\$24.8 million. } \\
\text { disbursed in } 1993 \text {. }\end{array}$ & $\begin{array}{l}\text { a) Modical Benefits Scheme. } \\
\text { b) Foreign currency levy. } \\
\text { c) Pre-paid income tax. } \\
\text { d) Deep Bay Development Company Account. }\end{array}$ & $\begin{array}{l}\text { a) EC\$56,066 a month } \\
\text { b) EC\$75,000 a month } \\
\text { c) EC } \$ 100,000 \text { a month } \\
\text { d) EC } \$ 18,688 \text { a month } \\
\text { (Remaining debt service of } \\
\text { EC\$80,037 a month is to be } \\
\text { paid from the Treasury's } \\
\text { Consolidated Fund.) }\end{array}$ \\
\hline $\begin{array}{l}\text { 12. Loan to settle arrears to } \\
\text { local supplier. }\end{array}$ & $\begin{array}{l}\text { Canadian Imperial Commercial Bank. } \\
\text { Amount of } 10 \text {-year loan was EC } \$ 6.4 \\
\text { million, disbursed in } 1993 \text {. }\end{array}$ & $\begin{array}{l}\text { Partial servicing from: } \\
\text { a) casino licensing fees; and } \\
\text { b) taxes owed by Hadeed Co. }\end{array}$ & $\begin{array}{l}\text { a) EC } \$ 300,000 \text { a year from } \\
\text { casino fees. } \\
\text { b) EC } \$ 200,000 \text { a year from } \\
\text { taxes owed by Hadeed Co. }\end{array}$ \\
\hline
\end{tabular}

Sources: Ministry of Finance: Antigua Port Authority: comercial banks; and Fund staff estimates. 
Table 12. Antigua and Barbuda: GDP by Sector of Origin at Current Prices

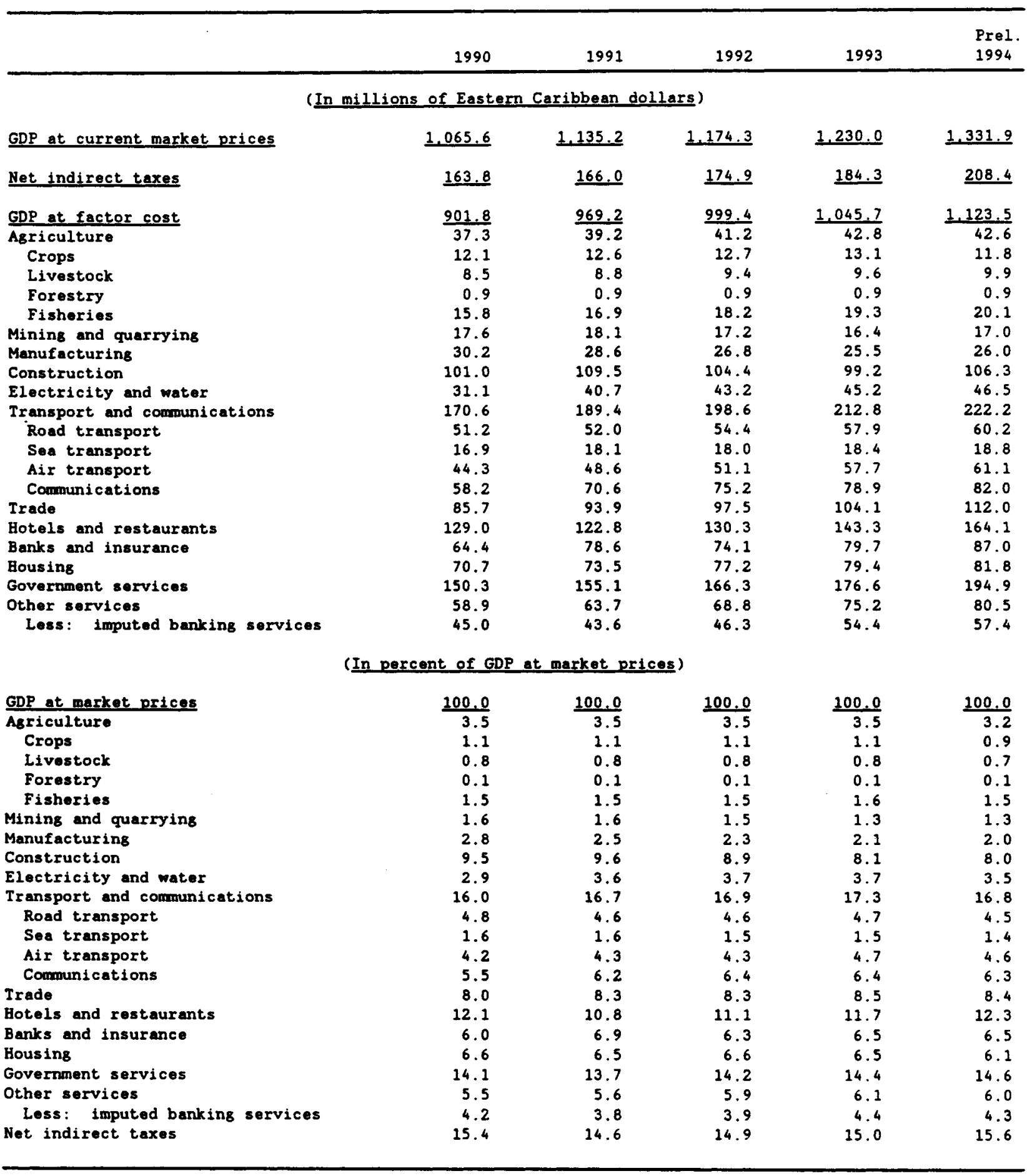

Sources: Statistics Division, Ministry of Finance; OECS; and Fund staff estimates. 
Table 13. Antigua and Berbuda: GDP by Sector of Origin at Constant 1977 Prices

\begin{tabular}{|c|c|c|c|c|c|}
\hline & 1990 & 1991 & 1992 & 1993 & $\begin{array}{l}\text { Prel. } \\
1994\end{array}$ \\
\hline \multicolumn{6}{|c|}{ (In millions of Eastern Caribben dollars) } \\
\hline GDP at factor cost & $\underline{362.0}$ & 378.1 & $\underline{382.4}$ & $\underline{395,2}$ & $\underline{412.0}$ \\
\hline Asriculture & 13.2 & 13.6 & 14.1 & 14.5 & 14.4 \\
\hline Crope & 3.5 & 3.7 & 3.8 & 3.9 & 3.5 \\
\hline Livestock & 5.5 & 5.6 & 5.7 & 5.9 & 6.0 \\
\hline Forestry & 0.4 & 0.4 & 0.4 & 0.4 & 0.4 \\
\hline Fishorios & 3.8 & 3.9 & 4.1 & 4.4 & 4.5 \\
\hline Mining and quarrying & 8.4 & 8.6 & 8.2 & 7.8 & 8.1 \\
\hline Manufecturing & 17.8 & 26.8 & 15.8 & 15.0 & 15.3 \\
\hline Construction & 35.8 & 38.8 & 37.0 & 35.2 & 36.6 \\
\hline Electricity and water & 11.3 & 22.0 & 12.6 & 13.1 & 13.5 \\
\hline Iraneport and communications & 89.0 & 97.3 & 100.7 & 106.8 & 111.5 \\
\hline Road transport & 22.4 & 22.9 & 22.3 & 23.7 & 24.6 \\
\hline Sea transport & 7.1 & 7.5 & 7.5 & 7.7 & 7.8 \\
\hline A1r transport & 21.6 & 21.0 & 21.5 & 24.2 & 25.7 \\
\hline Communication: & 37.8 & 45.9 & 49.4 & 51.2 & 53.3 \\
\hline Trade & 31.9 & 32.6 & 32.6 & 33.2 & 34.3 \\
\hline Botols and restaurants & 57.0 & 57.0 & 60.5 & 66.5 & 70.5 \\
\hline Banks and insurance & 25.4 & 27.0 & 26.1 & 27.4 & 28.6 \\
\hline Housing & 30.4 & 31.3 & 32.2 & 33.2 & 34.2 \\
\hline Govermment services & 33.3 & 34.0 & 33.2 & 34.1 & 36.1 \\
\hline Other services & 21.7 & 22.6 & 23.5 & 24.4 & 25.4 \\
\hline Less: imputed banking eervices & 13.2 & 13.4 & 14.1 & 16.0 & 16.6 \\
\hline \multicolumn{6}{|c|}{ (Annual percentake change) } \\
\hline GDP at factor cost & $\underline{3.5}$ & 4.4 & $\underline{1.1}$ & $\underline{3.4}$ & 4,2 \\
\hline Asriculture & 5.1 & 3.0 & 3.8 & $\overline{3.3}$ & -1.0 \\
\hline Crops & 9.0 & 4.0 & 4.9 & 2.9 & -11.9 \\
\hline Livestock & 2,4 & 2.2 & 2.5 & 1.9 & 2.6 \\
\hline Forestry & 2.5 & -2.4 & -2.5 & -2.6 & -2.6 \\
\hline Fisherios & 5.9 & 4.0 & 5.1 & 6.1 & 4.1 \\
\hline Mining and quarrying & -5.0 & 3.0 & -4.5 & -5.0 & 4.0 \\
\hline Manufacturing & 3.0 & -5.5 & -6.0 & -5.0 & 2.0 \\
\hline Construction & -10.0 & 8.4 & -4.6 & -5.0 & 4.0 \\
\hline Electricity and water & 1.6 & 6.2 & 5.0 & 4.6 & 3.0 \\
\hline Transport and communicetions & 4.2 & 9.3 & 3.5 & 6.1 & 4.3 \\
\hline Road transport & 4.6 & 1.9 & -2.3 & 6.0 & 4.0 \\
\hline Sea transport & 3.0 & 6.1 & -0.4 & 2.0 & 2.5 \\
\hline Air transport & 3.1 & -2.9 & 2.3 & 12.9 & 6.0 \\
\hline Conmunications & 5.0 & 21.3 & 7.6 & 3.7 & 4.0 \\
\hline Trade & 4.0 & 1.9 & -- & 1.9 & 3.5 \\
\hline Hotels and restaurants & 4.6 & -0.1 & 6.1 & 10.0 & 6.0 \\
\hline Banks and insurance & -11.5 & 6.1 & -3.2 & 5.0 & 4.5 \\
\hline Housing & 4.1 & 3.0 & 3.0 & 2.9 & 3.0 \\
\hline Government services & 3.8 & 2.1 & -2.4 & 2.7 & 6.0 \\
\hline Other services & 5.0 & 4.2 & 4.0 & 4.0 & 4.0 \\
\hline Less: Imputed benking services & -12.4 & 1.6 & 5.0 & 14.0 & 3.5 \\
\hline
\end{tabular}

Sources: Statistics Division, Ministry of Finance; OECS; and Fund staff estimates. 
Table 14. Antigua and Barbuda: Expenditure on GDP at Current Prices

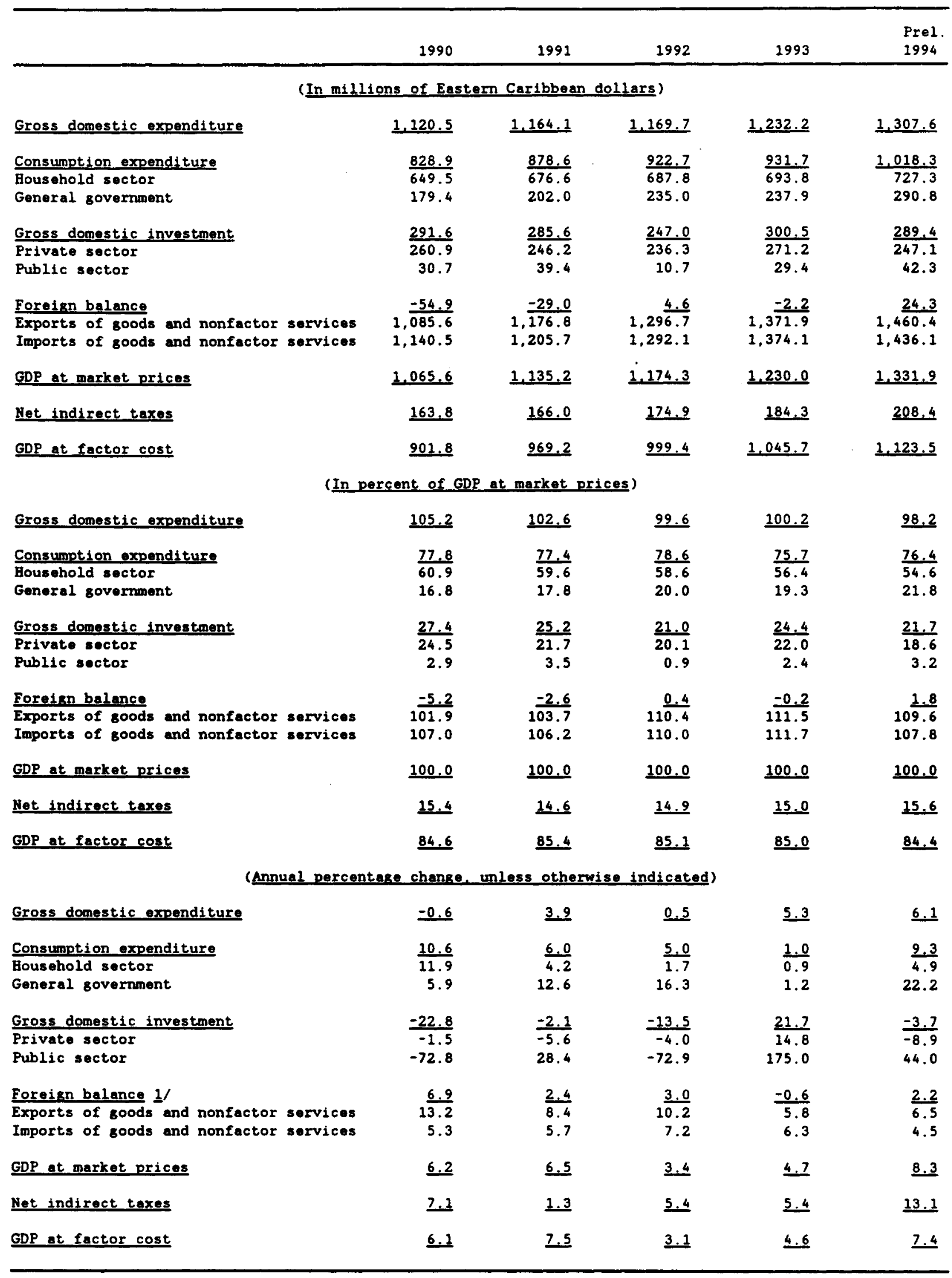

Sources: Statistical Appendix Iables 12, 22, and 29; and Fund staff estimates for household consumption and private sector investment.

1/ Change in percent of GDP in preceding year. 
Table 15. Antigue and Barbuda: Expenditure on GDP at Constant 1985 Prices $\underline{1}$ /

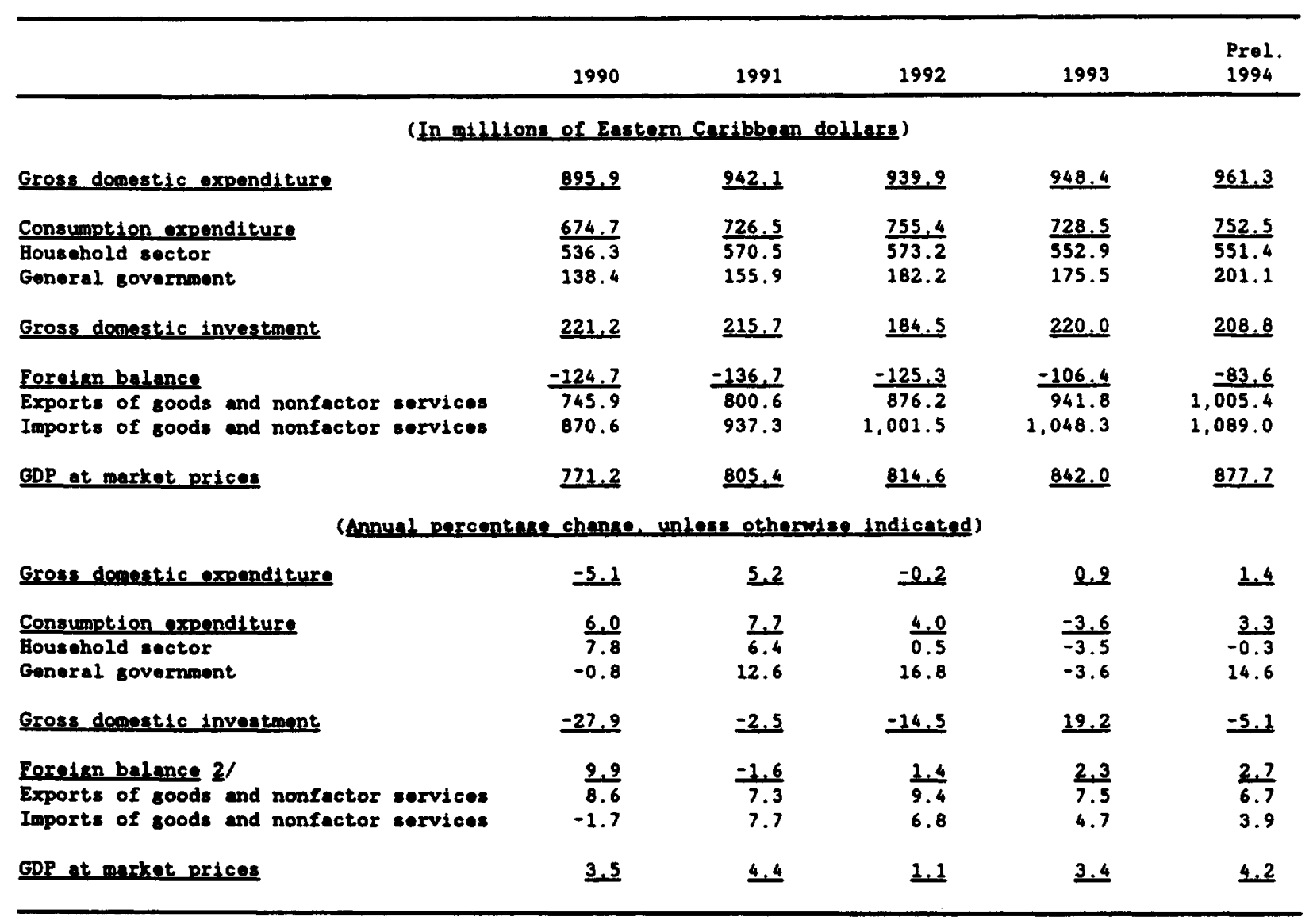

Sources: Statistical Appendix Iables 13, 22, and 29; and Fund staff estimates.

1/ Bousohold expenditure calculeted as residual; government consumption deflated by import prices and sovernment wase rates; exports deflated by an index of daily tourist expenditure; imports and investment deflated by export prices of Antigua and Barbuda's trading partners woighted by imports of Antigua and Barbuda.

2/ Change in percent of GDP in preceding year. 
Table 16. Antigua and Barbuda: Savings and Investment

\begin{tabular}{|c|c|c|c|c|c|}
\hline & 1990 & 1991 & 1992 & 1993 & $\begin{array}{l}\text { Prel. } \\
1994\end{array}$ \\
\hline \multicolumn{6}{|c|}{ (In millions of Eastern Caribbean dollars) } \\
\hline $\begin{array}{l}\text { Gross domestic savings } \\
\text { Private sector } \\
\text { Consolidated public sector }\end{array}$ & $\begin{array}{l}134.7 \\
163.7 \\
-29.0\end{array}$ & $\begin{array}{l}\frac{178.3}{216.3} \\
-38.0\end{array}$ & $\begin{array}{l}\frac{156.0}{212.9} \\
-57.0\end{array}$ & $\begin{array}{l}\frac{216.0}{256.4} \\
-40.4\end{array}$ & $\begin{array}{l}\frac{217.2}{264.2} \\
-47.0 \quad 1\end{array}$ \\
\hline Foreign savings & $\underline{156.9}$ & $\underline{107.3}$ & $\underline{91.0}$ & $\underline{84.5}$ & $\underline{72.1}$ \\
\hline Total savings & $\underline{291.6}$ & $\underline{285.6}$ & $\underline{247.0}$ & $\underline{300.5}$ & $\underline{289.4}$ \\
\hline $\begin{array}{l}\text { Gross domestic investment } \\
\text { Private sector } \\
\text { Public sector }\end{array}$ & $\begin{array}{r}\frac{291.6}{260.9} \\
30.7\end{array}$ & $\begin{array}{r}285.6 \\
246.2 \\
39.4\end{array}$ & $\begin{array}{r}\frac{247 \cdot 0}{236 \cdot 3} \\
10.7\end{array}$ & $\begin{array}{r}\frac{300.5}{271.2} \\
29.4\end{array}$ & $\begin{array}{r}\frac{289.4}{247.1} \\
42.3\end{array}$ \\
\hline \multicolumn{6}{|c|}{ (In percent of GDP at market prices) } \\
\hline $\begin{array}{l}\text { Gross domestic savings } \\
\text { Private sector } \\
\text { Consolidated public sector }\end{array}$ & $\begin{array}{l}\frac{12.6}{15.4} \\
-2.7\end{array}$ & $\begin{array}{l}\frac{15.7}{19.1} \\
-3.4\end{array}$ & $\begin{array}{l}\frac{13.3}{18.1} \\
-4.8\end{array}$ & $\begin{array}{l}\frac{17.6}{20.8} \\
-3.3\end{array}$ & $\begin{array}{l}\frac{16.3}{19.8} \\
-3.51 /\end{array}$ \\
\hline Foreign savings & 14.7 & $\underline{9.4}$ & $\underline{7.7}$ & $\underline{6.9}$ & $\underline{5.4}$ \\
\hline Total savings & 27.4 & 25.2 & $\underline{21.0}$ & $\underline{24.4}$ & $\underline{21.7}$ \\
\hline $\begin{array}{l}\text { Gross domestic investment } \\
\text { Private sector } \\
\text { Public sector }\end{array}$ & $\begin{array}{r}\frac{27.4}{24.5} \\
2.9\end{array}$ & $\begin{array}{r}\frac{25.2}{21.7} \\
3.5\end{array}$ & $\begin{array}{r}\frac{21.0}{20.1} \\
0.9\end{array}$ & $\begin{array}{r}\frac{24.4}{22.0} \\
2.4\end{array}$ & $\begin{array}{r}\frac{21.7}{18.6} \\
3.2\end{array}$ \\
\hline
\end{tabular}

Source: Statistical Appendix Tables 14, 22, and 29.

1/ Fiscal data are adjusted to exclude payments under the vocational training scheme (EC\$6.6 million in 1994), which in the national accounts are treated as government transfers to the private sector. 
Table 17. Antigua and Barbuda: Indicators of Construction Activity

\begin{tabular}{|c|c|c|c|c|c|}
\hline & 1990 & 1991 & 1992 & 1993 & $\begin{array}{l}\text { Prel } \\
1994\end{array}$ \\
\hline \multicolumn{6}{|c|}{ (In numbers, unless otherwise indicated) } \\
\hline $\begin{array}{l}\text { Number of building applications } \\
\text { New construction } \\
\text { Extension } \\
\text { Other }\end{array}$ & $\begin{array}{r}1.109 \\
788 \\
123 \\
198\end{array}$ & $\begin{array}{r}1.027 \\
765 \\
93 \\
169\end{array}$ & $\begin{array}{r}\frac{926}{705} \\
99 \\
122\end{array}$ & $\begin{array}{r}\frac{795}{591} \\
65 \\
139\end{array}$ & $\begin{array}{r}\frac{896}{658} \\
85 \\
153\end{array}$ \\
\hline \multicolumn{6}{|l|}{ Estimated value of applications } \\
\hline$\frac{\text { Total square footage approved }}{\text { (in thousands) }}$ & $\begin{array}{l}1.771 \\
\text { rcenta }\end{array}$ & $\begin{array}{l}1.842 \\
\text { change }\end{array}$ & $\underline{1.592}$ & $\underline{1.135}$ & 1.378 \\
\hline $\begin{array}{l}\text { Number of building applications } \\
\text { New construction } \\
\text { Extension } \\
\text { Other }\end{array}$ & $\begin{array}{l}-4.9 \\
-5.9 \\
-4.7 \\
-1.0\end{array}$ & $\begin{array}{l}\frac{-7.4}{-2.9} \\
-24.4 \\
-14.6\end{array}$ & $\begin{array}{r}\frac{-9.8}{-7.8} \\
6.5 \\
-27.8\end{array}$ & $\begin{array}{r}\frac{-14.1}{-16.2} \\
-34.3 \\
13.9\end{array}$ & $\begin{array}{l}\frac{12.7}{11.3} \\
30.8 \\
10.1\end{array}$ \\
\hline Estimated value of applications & -8.9 & 27.5 & -7.5 & -44.2 & $\underline{38.0}$ \\
\hline Total square footage approved & -5.6 & $\underline{4.0}$ & -13.6 & -28.7 & $\underline{21.4}$ \\
\hline
\end{tabular}

Source: Development Control Authority. 
Table 18. Antigua and Barbuda: Electricity Generation, Consumption, and Tariffs

\begin{tabular}{|c|c|c|c|c|c|}
\hline & 1990 & 1991 & 1992 & 1993 & $\begin{array}{l}\text { Prel. } \\
1994\end{array}$ \\
\hline \multicolumn{6}{|c|}{ (In millions of kilowatt-hours) } \\
\hline $\begin{array}{l}\text { Units generated } \\
\text { Units consumed }\end{array}$ & $\begin{array}{r}101.0 \\
91.5\end{array}$ & $\begin{array}{l}120.0 \\
111.0\end{array}$ & $\begin{array}{l}116.2 \\
106.4\end{array}$ & $\begin{array}{l}112.8 \\
102.3\end{array}$ & $\begin{array}{l}117.5 \\
105.7\end{array}$ \\
\hline \multicolumn{6}{|c|}{ (Annual percentage change) } \\
\hline $\begin{array}{l}\text { Units generated } \\
\text { Units consumed }\end{array}$ & $\begin{array}{l}8.4 \\
7.5\end{array}$ & $\begin{array}{l}18.8 \\
21.3\end{array}$ & $\begin{array}{l}-3.2 \\
-4.1\end{array}$ & $\begin{array}{l}-2.9 \\
-3.8\end{array}$ & $\begin{array}{l}4.2 \\
3.3\end{array}$ \\
\hline \multicolumn{6}{|c|}{ (In Eastern Caribbean dollars) } \\
\hline \multicolumn{6}{|l|}{ Domestic tariff } \\
\hline Rate per kwh & 0.4 & 0.4 & 0.4 & 0.4 & 0.4 \\
\hline Minimum charge per month & 8.0 & 8.0 & 8.0 & 25.0 & 25.0 \\
\hline \multicolumn{6}{|l|}{ Commercial tariff } \\
\hline Rate per kwh & 0.45 & 0.45 & 0.45 & 0.44 & 0.44 \\
\hline Minimum charge per month & 45 & 45 & 45 & 45 & 45 \\
\hline \multicolumn{6}{|l|}{ Industrial tariff } \\
\hline Rate per kwh & 0.45 & 0.45 & 0.45 & 0.43 & 0.43 \\
\hline Minimum charge per month & 45 & 45 & 45 & 45 & 45 \\
\hline
\end{tabular}

Source: Antigua Public Utilities Authority. 
Table 19. Antigua and Barbuda: Consumer Prices

(Annual percentage change)

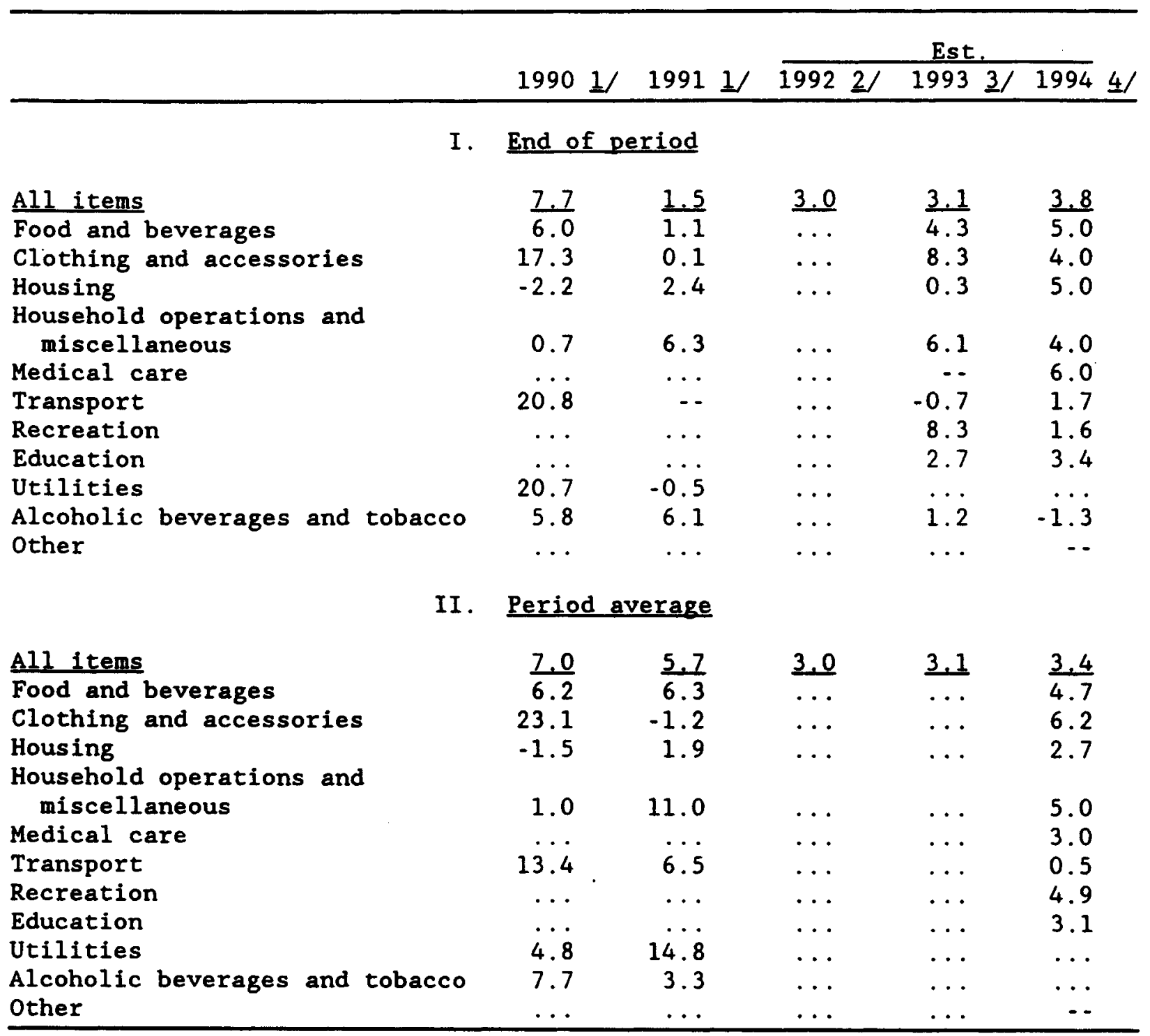

Sources: Statistics Division, Ministry of Finance; and Fund staff estimates.

1/ Based on unpublished consumer price index compiled by the Statistics Division, Ministry of Finance. The index was discontinued after December 1991 and was replaced by a revised index, beginning September 1993, that is based on methodology developed with the assistance of the Fund's Statistics Department.

2/ Fund staff estimate.

3/ The 1993 end-of-period estimate is based on an annualization of monthly average changes in the period October-December 1993.

4/ The 1994 end-of-period estimate is based on the percentage change from October-December 1993 to the corresponding period of 1994. 
Table 20. Antigua and Barbuda: Retail Prices of Petroleum Products

(In U.S. dollars per imperial gallon at end of period. unless otherwise indicated)

$1990-94$

Gasoline

2.54

Kerosene

1.53

Diesel

2.43

Propane (in U.S. dollars per pound)

0.4

Sources: Statistics Division, Ministry of Finance; and West Indies Oil Company. 
Table 21. Antigua and Barbuda: Estimates of Population and Labor Force

\begin{tabular}{|c|c|c|c|c|c|}
\hline & 1990 & 1991 & 1992 & $\begin{array}{l}\text { Prel. } \\
1993\end{array}$ & $\begin{array}{l}\text { Est. } \\
1994\end{array}$ \\
\hline \multicolumn{6}{|c|}{ (In numbers) } \\
\hline $\begin{array}{l}\text { Total midyear population } \\
\text { Total births } \\
\text { Total deaths } \\
\text { Natural population increase } \\
\text { Net immigration } 1 / \\
\text { Net total population increase }\end{array}$ & $\begin{array}{r}63,839 \\
1,288 \\
404 \\
884 \\
-833 \\
51\end{array}$ & $\begin{array}{r}63.896 \\
1,178 \\
412 \\
766 \\
-709 \\
57\end{array}$ & $\begin{array}{r}63.939 \\
1,257 \\
416 \\
841 \\
-798 \\
43\end{array}$ & $\begin{array}{r}64.049 \\
1,253 \\
434 \\
819 \\
-709 \\
110\end{array}$ & $\begin{array}{r}64.166 \\
1,217 \\
415 \\
802 \\
-685 \\
117\end{array}$ \\
\hline \multicolumn{6}{|c|}{ (Annual percentage change) } \\
\hline $\begin{array}{l}\text { Crude birth rate } \\
\text { Crude death rate } \\
\text { Rate of natural increase } \\
\text { Net immigration } \\
\text { Net total population increase }\end{array}$ & $\begin{array}{r}1.4 \\
0.5 \\
0.9 \\
-1.3 \\
0.1\end{array}$ & $\begin{array}{r}1.8 \\
0.6 \\
1.2 \\
-1.1 \\
0.1\end{array}$ & $\begin{array}{r}2.0 \\
0.7 \\
1.3 \\
-1.2 \\
0.1\end{array}$ & $\begin{array}{r}2.0 \\
0.7 \\
1.3 \\
-1.1 \\
0.2\end{array}$ & $\begin{array}{r}1.9 \\
0.6 \\
1.2 \\
-1.1 \\
0.2\end{array}$ \\
\hline \multicolumn{6}{|c|}{ (In numbers) } \\
\hline $\begin{array}{l}\text { Total active labor force } \\
\text { Antiguan active labor } \\
\text { force } 2 / \\
\text { Total immigrants } 3 /\end{array}$ & $\begin{array}{r}28,383 \\
21,156 \\
7,227\end{array}$ & $\begin{array}{r}28,671 \\
21,175 \\
7,496\end{array}$ & $\begin{array}{r}28,878 \\
21,189 \\
7,689\end{array}$ & $\begin{array}{r}28,247 \\
21,226 \\
7,021\end{array}$ & $\begin{array}{r}28,495 \\
21,264 \\
7,231\end{array}$ \\
\hline \multicolumn{6}{|c|}{ (Annual percentage change) } \\
\hline Total active labor force & $\underline{4.9}$ & 1.0 & $\underline{0.7}$ & -2.2 & $\underline{0.9}$ \\
\hline
\end{tabular}

Sources: Statistics Division, Ministry of Finance; and Fund staff estimates.

1/ Reflects net emigration of citizens of Antigua and Barbuda; calculated as a residual.

2/ Assumed to be one third of Antigua and Barbuda's population.

3/ Based on work permits outstanding at the end of the year. 
Table 22. Antigua and Barbuda: Operations of the Consolidated Public Sector

(In millions of Eastern Caribbean dollars)

\begin{tabular}{|c|c|c|c|c|c|}
\hline & 1990 & 1991 & 1992 & 1993 & $\begin{array}{l}\text { Prel. } \\
1994\end{array}$ \\
\hline \multicolumn{6}{|c|}{ I. Consolidated Public Sector } \\
\hline Total revenue and grants & 270.5 & 275.5 & 293.2 & 313.4 & 352.8 \\
\hline Current revenue & 256.7 & $\overline{261.2}$ & $\overline{282.0}$ & $\overline{301.6}$ & $\overline{343.6}$ \\
\hline Capital revenue & 8.5 & 4.4 & 3.5 & 2.4 & 3.0 \\
\hline Capital grants & 5.3 & 9.9 & 7.7 & 9.4 & 6.2 \\
\hline Totel expenditure and net lending & 316.4 & $\underline{338.6}$ & $\underline{349.6}$ & $\underline{371.4}$ & 439.5 \\
\hline Current expenditure $1 /$ & 285.7 & 299.2 & 339.0 & 342.0 & 397.2 \\
\hline of which: interest on external debt & 72.5 & 69.3 & 78.7 & 72.8 & 73.6 \\
\hline cash & 8.2 & 7.6 & 8.1 & 10.8 & 8.6 \\
\hline arrears & 64.3 & 61.7 & 70.6 & 62.1 & 65.0 \\
\hline Capital expenditure and net lending & 30.7 & 39.4 & 10.7 & 29.4 & 42.3 \\
\hline Fixed capital expenditure & 30.4 & 39.4 & 10.3 & 25.9 & 32.3 \\
\hline Purchase of land & 1.3 & -- & 0.4 & 3.5 & $\therefore$ \\
\hline Change in stocks & -1.0 & -- & -- & - & -- \\
\hline Net lending & -- & -- & -- & -- & 10.0 \\
\hline Current account balance & -29.0 & -38.0 & -57.0 & -40.4 & -53.6 \\
\hline Overall balance & -45.9 & -63.1 & $\underline{-56.4}$ & -58.0 & $\underline{-86.7}$ \\
\hline$\frac{\text { Financins }}{\text { External (net) }}$ & $\frac{45.9}{50.1}$ & $\frac{63.1}{70.0}$ & $\frac{56.4}{55.7}$ & $\frac{58.0}{47.5}$ & $\frac{86.7}{55.3}$ \\
\hline Drawings & 10.4 & 22.5 & 0.6 & -- & 4.4 \\
\hline Amortization & -96.3 & -82.3 & -85.6 & -61.9 & -44.1 \\
\hline Cash & -23.9 & -14.1 & -15.5 & -14.6 & -14.1 \\
\hline Arrears & -72.5 & -68.2 & -70.2 & -47.3 & -30.0 \\
\hline Change in foreign assets & -0.8 & -- & -- & - & -- \\
\hline Rescheduled arrears $\underline{2}$ & 59.1 & -- & -- & -- & -- \\
\hline Net change in arrears $\underline{3}$ / & 77.7 & 129.9 & 140.8 & 109.4 & 95.0 \\
\hline Principal & 38.1 & 68.2 & 70.2 & 47.3 & 30.0 \\
\hline Interest & 39.6 & 61.7 & 70.6 & 62.1 & 65.0 \\
\hline Domestic & -4.2 & -6.9 & 0.7 & 10.5 & 31.4 \\
\hline Net ECCB borrowing & 7.2 & 1.5 & -8.6 & 2.3 & 0.1 \\
\hline Coumercial banks & -15.0 & -16.0 & 6.7 & 22.1 & 10.2 \\
\hline \multicolumn{6}{|c|}{ II. General Government } \\
\hline Total revenue and grants & $\underline{269.1}$ & 263.0 & $\underline{292.7}$ & 299.1 & 330.4 \\
\hline Current revenue & $\overline{256.7}$ & 255.0 & 281.5 & 290.4 & $\overline{321.2}$ \\
\hline Capital revenue & 7.1 & 4.4 & 3.5 & 2.4 & 3.0 \\
\hline Capital grants & 5.3 & 3.6 & 7.7 & 6.3 & 6.2 \\
\hline Total expenditure and net lending & $\underline{262.8}$ & 274.8 & $\underline{287.2}$ & $\underline{294.8}$ & $\underline{365.2}$ \\
\hline Current expenditure & $\overline{243.2}$ & $\overline{241.8}$ & 276.5 & $\overline{268.5}$ & $\overline{322.9}$ \\
\hline of which: transfers to public enterprises & 0.7 & 0.8 & 1.0 & 1.3 & 1.0 \\
\hline Capital expenditure and not lending & 19.6 & 33.1 & 10.7 & 26.3 & 42.3 \\
\hline Fixed capital expenditure & 16.1 & 33.1 & 10.3 & 22.8 & 32.3 \\
\hline Purchase of land & 1.3 & -- & 0.4 & 3.5 & - \\
\hline Net lending & -- & -- & -- & -- & 10.0 \\
\hline Capital transfors to public onterprises & 2.2 & -- & -- & -- & -- \\
\hline Current account belence & 13.5 & $\underline{13.2}$ & $\underline{5.0}$ & 21.9 & -1.7 \\
\hline Overall balance & $\underline{6.4}$ & -11.9 & $\underline{5.5}$ & $\underline{4.4}$ & -34.8 \\
\hline
\end{tabular}


Table 22. Antigue and Barbuda: Operations of the Consolidated Public Sector (Continued)

(In millions of Eastern Caribbean dollars)

\begin{tabular}{|c|c|c|c|c|c|}
\hline & 1990 & 1991 & 1992 & 1993 & 1994 \\
\hline \multicolumn{6}{|c|}{ al Gove } \\
\hline Total revenue and grants & 235.9 & 229.5 & 252.6 & 255.5 & 285.2 \\
\hline Current revenue & 223.5 & $\overline{221.5}$ & $\overline{241.4}$ & $\overline{246.8}$ & $\overline{276.0}$ \\
\hline Capital revenue & 7.1 & 4.4 & 3.5 & 2.4 & 3.0 \\
\hline Capital grants & 5.3 & 3.6 & 7.7 & 6.3 & 6.2 \\
\hline Total expenditure and not lendins & 255.0 & 264.2 & $\underline{278.2}$ & 282.0 & 350.7 \\
\hline Current expenditure $1 /$ & $\overline{235.6}$ & $\overline{231.1}$ & 267.5 & $\overline{255.7}$ & $\overline{308.4}$ \\
\hline \multicolumn{6}{|l|}{ of which: } \\
\hline Contributions to Social Security Scheme & 5.0 & 5.4 & 5.9 & 6.1 & 7.0 \\
\hline Cash & -- & -- & -- & $\rightarrow$ & -- \\
\hline Arrears & 5.0 & 5.4 & 5.9 & 6.1 & 7.0 \\
\hline Contributions to Medical Benefits Scheme & 2.1 & 2.2 & 2.8 & 3.0 & 3.3 \\
\hline Cash & -- & - & -- & -- & -- \\
\hline Arrears & 2.1 & 2.2 & 2.8 & 3.0 & 3.3 \\
\hline Interest arrears Social Security Scheme & 2.5 & 2.6 & 3.0 & 3.7 & 4.4 \\
\hline Interest on external debt & 15.8 & 15.3 & 17.6 & 18.3 & 19.4 \\
\hline Cash & 5.3 & 7.1 & 7.3 & 10.3 & 7.9 \\
\hline Arrears & 10.5 & 8.2 & 10.4 & B. 0 & 11.5 \\
\hline Iransfers to rest of public sector & 0.7 & 0.8 & 1.0 & 1.3 & 1.0 \\
\hline Capital expenditure and net lending & 19.4 & 33.1 & 10.7 & 26.3 & 42.3 \\
\hline Fixed capital expenditure & 15.9 & 33.1 & 10.3 & 22.8 & 32.3 \\
\hline Purchase of land & 1.3 & -- & 0.4 & 3.5 & -- \\
\hline Net lending & -- & -- & -- & -- & 10.0 \\
\hline Capital transfers to public enterprises & 2.2 & -- & -- & -- & -- \\
\hline Current account balance & -12.1 & -9.7 & -26.1 & -8.9 & -32.4 \\
\hline Overall balince & $-19,1$ & -34.8 & -25.6 & -26.5 & -65.5 \\
\hline Financins & 19.1 & 34.7 & 24.0 & 26.5 & 65.5 \\
\hline External (net) & $\overline{20.4}$ & -0.2 & $-\overline{23.6}$ & $-\overline{24.0}$ & $-\overline{18.7}$ \\
\hline External borrowing (net) & -16.5 & -0.2 & -23.6 & -24.0 & -18.7 \\
\hline Drawings & 10.4 & 22.5 & 0.6 & -- & 4.4 \\
\hline Amortization & -27.0 & -22.7 & -24.2 & -24.0 & -23.1 \\
\hline Cash & -21.1 & -13.8 & -13.3 & -13.9 & -13.2 \\
\hline Arrears & -5.8 & -8.9 & -10.9 & -10.1 & -9.9 \\
\hline Change in foreign essets & -0.8 & -- & -- & -- & - \\
\hline Rescheduled arrears & 37.7 & -- & -- & -- & -- \\
\hline Domestic & 12.7 & -5.2 & 11.7 & 30.4 & 28.6 \\
\hline ECCB (net) & 7.2 & 1.5 & -8.6 & 2.3 & 0.1 \\
\hline Commercial banks & -6.2 & -6.4 & 15.0 & 21.0 & 6.0 \\
\hline Social Security Scheme & 13.6 & 5.2 & -- & - & -- \\
\hline Rest of public sector & 0.1 & -1.7 & -- & -- & -- \\
\hline Other private sector (bills and bonds) & -2.0 & -3.8 & 5.3 & 4.9 & 5.0 \\
\hline Change in arrears & -14.0 & 40.2 & 35.9 & 20.1 & 55.6 \\
\hline External (net increase) & -21.4 & 17.1 & 21.2 & 18.1 & 21.4 \\
\hline Principal & -18.8 & 8.9 & 10.9 & 10.1 & 9.9 \\
\hline Interest & -2.6 & 8.2 & 10.4 & B. 0 & 11.5 \\
\hline Domestic & 7.4 & 23.1 & 14.7 & 2.0 & 34.2 \\
\hline Social Securlty Scheme & -1.8 & 4.9 & 11.2 & 12.1 & 13.5 \\
\hline Medical Benefits Scheme & 4.3 & 4.5 & 5.5 & 5.9 & 6.6 \\
\hline Interest & -- & 3.9 & 1.6 & 0.4 & 4.3 \\
\hline Floating debt & 4.9 & 9.8 & -3.7 & -5.8 & 7.8 \\
\hline Vouchers & 9.5 & 9.8 & -3.7 & -5.8 & 1.7 \\
\hline Checks & -4.6 & -- & -- & -- & 6.1 \\
\hline Other & - & -- & 0.1 & -10.6 & 2.0 \\
\hline
\end{tabular}


Table 22. Antigua and Barbuda: Operations of the Consolidated Public Sector (Continued)

(In millions of Eastern Caribbean dollars)

\begin{tabular}{|c|c|c|c|c|c|}
\hline & 1990 & 1991 & 1992 & 1993 & $\begin{array}{l}\text { Prel. } \\
1994\end{array}$ \\
\hline II. 2 & \multicolumn{5}{|c|}{ Social Security Scheme } \\
\hline Total revenue and grants & 34.8 & 35.2 & 38.3 & 41.9 & 43.7 \\
\hline Current revenue & 34.8 & $\overline{35.2}$ & $\overline{38.3}$ & $\overline{41.9}$ & $\overline{43.7}$ \\
\hline Contributions & 28.0 & 28.8 & 29.7 & 33.0 & 34.5 \\
\hline \multirow{2}{*}{$\begin{aligned} \text { Of which: central government } \\
\text { central government }\end{aligned}$} & -- & -- & -- & -- & -- \\
\hline & 8.0 & 8.2 & 8.3 & 8.5 & 9.8 \\
\hline \multirow{2}{*}{$\begin{array}{l}\text { Interest } \\
\text { of which: contral government arrears }\end{array}$} & 5.2 & 5.2 & 7.4 & 7.6 & 7.9 \\
\hline & 2.5 & 2.6 & 3.0 & 3.7 & 4.4 \\
\hline Other & 1.6 & 1.2 & 1.2 & 1.3 & 1.3 \\
\hline Total expenditure & 13.1 & 12.9 & 13.3 & 16.3 & 20.4 \\
\hline Current expenditure & 12.9 & $\overline{12.9}$ & 13.3 & $\overline{16.3}$ & $\overline{20.4}$ \\
\hline & 8.8 & 9.3 & 9.4 & 11.8 & 15.6 \\
\hline Wages and salaries & 2.1 & 2.2 & 2.3 & 2.7 & 2.8 \\
\hline Other & 2.0 & 1.4 & 1.6 & 1.8 & 2.0 \\
\hline Capital exponditure & 0.2 & -- & -- & -- & -- \\
\hline Current account balance & $\underline{21.9}$ & $\underline{22.3}$ & $\underline{25.0}$ & $\underline{25.6}$ & $\underline{23.3}$ \\
\hline Overall balance & $\underline{21.7}$ & $\underline{22.3}$ & $\underline{25.0}$ & $\underline{25.6}$ & 23.3 \\
\hline \multicolumn{6}{|c|}{ edical Benefits Scheme } \\
\hline Total revenue and grants & $\underline{13.2}$ & 13.6 & 18.7 & 19.9 & $\underline{22.2}$ \\
\hline Current revenue & $\overline{13.2}$ & $\overline{13.6}$ & $\overline{18.7}$ & $\overline{19.9}$ & $\overline{22.2}$ \\
\hline \multirow{2}{*}{$\begin{array}{l}\text { Contributions } \\
\text { Of which: Central Government }\end{array}$} & 12.7 & 13.0 & 18.1 & 19.3 & 21.6 \\
\hline & 4.3 & 4.5 & 5.6 & 5.9 & 6.6 \\
\hline Interest & 0.1 & 0.1 & -- & 0.1 & 0.1 \\
\hline Other & 0.4 & 0.5 & 0.6 & 0.5 & 0.5 \\
\hline Total expenditure & 9.5 & 13.0 & 12.4 & 14.5 & 17.5 \\
\hline Current expenditure & $\overline{9.5}$ & $\overline{13.0}$ & $\overline{12.4}$ & $\overline{14.5}$ & $\overline{17.5}$ \\
\hline $\begin{array}{l}\text { Benefits } \\
\text { Wages and salaries }\end{array}$ & 1.4 & 0.6 & 0.7 & 0.8 & 0.9 \\
\hline Wages and salaries & 1.2 & 1.5 & 1.5 & 1.5 & 1.6 \\
\hline Other & 6.9 & 10.9 & 10.2 & 12.2 & 14.9 \\
\hline Capital expenditure & $=$ & $=$ & $=$ & $=$ & $=$ \\
\hline Current account balance & $\underline{3.7}$ & $\underline{0.6}$ & $\underline{6.3}$ & $\underline{5.4}$ & $\underline{4.7}$ \\
\hline Overall balance & $\underline{3.7}$ & $\underline{0.6}$ & $\underline{6.3}$ & $\underline{5.4}$ & $\underline{4.7}$ \\
\hline
\end{tabular}


Iable 22. Antigua and Barbuda: Operations of the Consolidated Public Sector (Continued)

(In millions of Eastern Caribbean dollars)

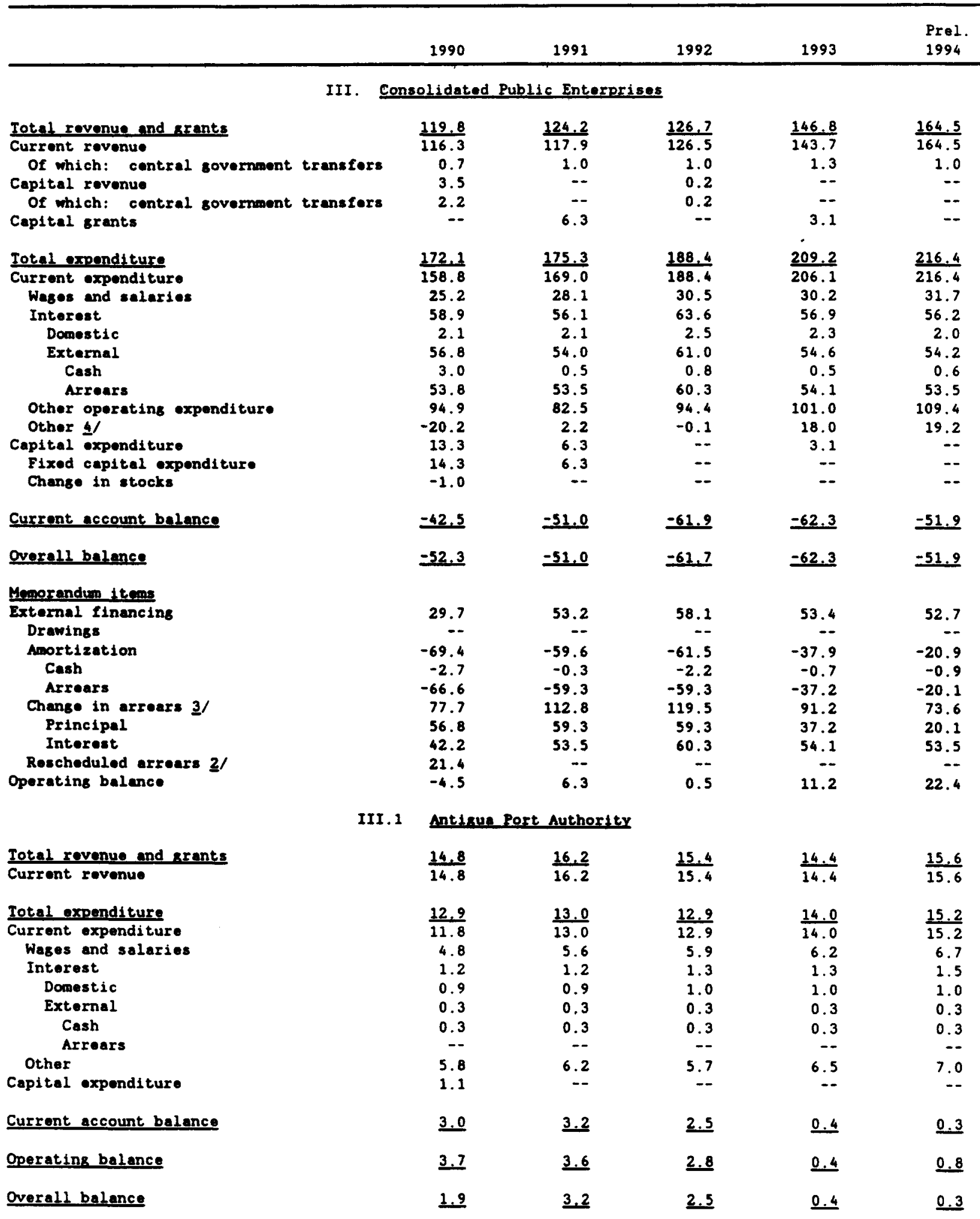


Table 22. Antigua and Barbuda: Operations of the Consolidated Public Sector (Continued)

(In millions of Eastern Caribbean dollars)

\begin{tabular}{|c|c|c|c|c|c|}
\hline & 1990 & 1991 & 1992 & 1993 & $\begin{array}{l}\text { Prel. } \\
1994\end{array}$ \\
\hline \multicolumn{6}{|l|}{ Memorandum items } \\
\hline External financing & -- & -- & -- & -- & -- \\
\hline Amortization & -0.4 & -0.4 & -0.4 & -0.4 & -0.4 \\
\hline Change in arrears & 0.4 & 0.4 & 0.4 & 0.4 & 0.4 \\
\hline \multirow[t]{2}{*}{ Principal } & 0.4 & 0.4 & 0.4 & 0.4 & 0.4 \\
\hline & \multicolumn{5}{|c|}{ Antigua Public Utilities Authority } \\
\hline Totel revenue and grants & $\underline{63.0}$ & 68.2 & 67.7 & 88.2 & 102.1 \\
\hline Current revenue & $\overline{60.8}$ & $\overline{61.9}$ & $\overline{67.5}$ & $\overline{85.1}$ & $\overline{102.1}$ \\
\hline Capital revenue and government transfers & 2.2 & -- & 0.2 & -- & -- \\
\hline Capital grants & -- & 6.3 & -- & 3.1 & -- \\
\hline Total expenditure & 107.7 & 86.7 & 95.1 & 102.6 & 107.4 \\
\hline Current expenditure & 98.4 & $\overline{80.4}$ & $\overline{95.1}$ & 99.5 & $\overline{107.4}$ \\
\hline Wages and salaries & 19.5 & 21.4 & 23.3 & 22.5 & 23.4 \\
\hline Interest & 25.9 & 18.7 & 19.2 & 19.6 & 21.9 \\
\hline Domestic & 0.2 & 0.1 & 0.1 & 0.1 & 0.1 \\
\hline External & 25.7 & 18.6 & 19.1 & 19.5 & 21.8 \\
\hline Cash & 2.3 & -- & -- & -- & 0.2 \\
\hline Arrears & 23.4 & 18.6 & 19.1 & 19.5 & 21.6 \\
\hline Other & 53.0 & 40.3 & 52.6 & 57.4 & 62.1 \\
\hline Capital expenditure & 9.3 & 6.3 & -- & 3.1 & -- \\
\hline Fixed capital expenditure & 10.3 & 6.3 & -- & 3.1 & -- \\
\hline Change in stocks & -1.0 & -- & -- & -- & - \\
\hline Current account balance & -37.6 & -18.5 & -27.6 & -14.4 & -5.3 \\
\hline Operating balance & -11.7 & $\underline{0.2}$ & -8.4 & $\underline{5.2}$ & $\underline{16.6}$ \\
\hline Overall balance & -44.7 & -18.5 & -27.4 & -14.4 & -5.3 \\
\hline \multicolumn{6}{|l|}{ Memorandum 1tems } \\
\hline External financing & 42.7 & 18.6 & 19.1 & 19.5 & 21.6 \\
\hline Drawings & -- & -- & -- & -. & - \\
\hline Amortization & -27.4 & -18.7 & -18.1 & -13.3 & -12.7 \\
\hline Cash & -2.0 & - & - & -- & - \\
\hline Arrears & -25.4 & -18.7 & -18.1 & -13.3 & -12.7 \\
\hline Change in arrears 1 / & 48.7 & 37.3 & 37.2 & 32.8 & 34.3 \\
\hline Rescheduled arrears $\underline{2}$ & 21.4 & -- & -- & -- & -- \\
\hline
\end{tabular}


Table 22. Antigua and Barbuda: Operations of the Consolidated Public Sector (Continued)

(In millions of Eastern Caribbean dollars)

\begin{tabular}{|c|c|c|c|c|c|c|}
\hline & & 1990 & 1991 & 1992 & 1993 & $\begin{array}{l}\text { Prel. } \\
1994\end{array}$ \\
\hline & III. 3 & \multicolumn{5}{|c|}{ Central Marketing Corporation } \\
\hline \multicolumn{2}{|l|}{ Total revenue and grants } & 10.2 & 10.5 & 10.9 & 10.8 & 11.4 \\
\hline \multicolumn{2}{|l|}{ Current revenue } & $\overline{10.2}$ & $\overline{10.5}$ & $\overline{10.9}$ & $\overline{10.8}$ & $\overline{11.4}$ \\
\hline Of which: central government & transfers & -- & -- & -- & -- & - \\
\hline \multicolumn{2}{|l|}{ Total expenditure } & 9.8 & 10.5 & 10.4 & 10.1 & 10.7 \\
\hline \multicolumn{2}{|l|}{ Current expenditure } & $\overline{9.0}$ & $\overline{10.5}$ & $\overline{10.4}$ & $\overline{10.1}$ & $\overline{10.7}$ \\
\hline \multicolumn{2}{|l|}{ Wages and salaries } & 0.6 & 0.6 & 0.7 & 0.7 & 0.7 \\
\hline \multirow{2}{*}{\multicolumn{2}{|c|}{$\begin{array}{l}\text { Interest } \\
\text { Other }\end{array}$}} & 0.1 & 0.3 & 0.3 & 0.2 & 0.1 \\
\hline & & 8. 3 & 9.5 & 9.5 & 9.1 & 9.9 \\
\hline \multicolumn{2}{|l|}{ Capital expenditure } & 0.8 & - & -- & -- & -- \\
\hline \multicolumn{2}{|l|}{ Current account balance } & $\underline{1.2}$ & $\underline{0.1}$ & $\underline{0.5}$ & $\underline{0.8}$ & $\underline{0.7}$ \\
\hline \multicolumn{2}{|l|}{ Operating balance } & 1.3 & $\underline{0.4}$ & $\underline{0.8}$ & $\underline{1.0}$ & $\underline{0.8}$ \\
\hline \multicolumn{2}{|l|}{ Overall balance } & $\underline{0.4}$ & $\underline{0.1}$ & $\underline{0.5}$ & $\underline{0.8}$ & $\underline{0.7}$ \\
\hline \multicolumn{7}{|c|}{ ep Bay Development Company } \\
\hline \multicolumn{2}{|l|}{ Total rovenue } & 27.7 & 25.0 & 27.5 & 27.1 & 28.3 \\
\hline \multicolumn{2}{|l|}{ Current revenue } & $\overline{26.4}$ & 25.0 & 27.5 & 27.1 & $\overline{28.3}$ \\
\hline \multirow{2}{*}{\multicolumn{2}{|c|}{$\begin{array}{l}\text { Hotel } \\
\text { Villas (rentals) }\end{array}$}} & 22.5 & 20.9 & 23.5 & 23.0 & 24.1 \\
\hline & & 3.9 & 4.1 & 4.0 & 4.1 & 4.2 \\
\hline \multicolumn{2}{|l|}{ Capital revenue (villa sales) } & 1.4 & -- & -- & -- & -- \\
\hline \multicolumn{2}{|l|}{ Total expenditure } & 54.3 & 54.2 & 60.2 & $\underline{55.7}$ & 55.5 \\
\hline \multirow{2}{*}{\multicolumn{2}{|c|}{ Current expenditure }} & $\overline{52.2}$ & $\overline{54.2}$ & $\overline{60.2}$ & $\overline{55.7}$ & $\overline{55.5}$ \\
\hline \multirow{2}{*}{\multicolumn{2}{|c|}{$\begin{array}{l}\text { Operating exponditure } \\
\text { Interest paymonts }\end{array}$}} & 26.0 & 24.5 & 24.6 & 25.9 & 27.9 \\
\hline & & 26.2 & 29.7 & 35.6 & 29.8 & 27.6 \\
\hline \multicolumn{2}{|l|}{ Domestic } & 0.8 & 0.7 & 1.1 & 0.9 & 0.7 \\
\hline \multicolumn{2}{|l|}{$\begin{array}{c}\text { External } \\
\text { Cash }\end{array}$} & 25.4 & 29.0 & 34.5 & 29.0 & 26.9 \\
\hline \multirow{2}{*}{\multicolumn{2}{|c|}{$\begin{array}{l}\text { Cash } \\
\text { Arrears }\end{array}$}} & -- & -- & $\cdots$ & $\cdots$ & - \\
\hline & & 25.4 & 29.0 & 34.5 & 29.0 & 26.9 \\
\hline \multicolumn{2}{|l|}{ Capital expenditure } & 2.1 & -- & -- & -- & -- \\
\hline \multicolumn{2}{|l|}{ Current account balance } & -25.8 & -29.3 & -32.7 & -28.6 & -27.2 \\
\hline \multicolumn{2}{|l|}{ Operatins balance } & $\underline{0.4}$ & $\underline{0.5}$ & $\underline{2.8}$ & $\underline{1.2}$ & $\underline{0.4}$ \\
\hline \multicolumn{2}{|l|}{ Overall balance } & $\underline{-26.5}$ & $-29,3$ & -32.7 & -28.6 & -27.2 \\
\hline Memorandum items & & & & & & \\
\hline External finencing & & 25.4 & 29.0 & 34.5 & 29.0 & 26.9 \\
\hline Drawings & & -- & -- & -- & -- & -- \\
\hline Amortization & & -33.6 & -32.7 & -34.7 & -16.4 & -- \\
\hline Arrears & & -33.6 & -32.7 & -34.7 & -16.4 & -. \\
\hline Change in arrears & & 58.9 & 61.7 & 69.3 & 45.4 & 26.9 \\
\hline Principal & & 33.6 & 32.7 & 34.7 & 16.4 & -- \\
\hline Interest & & 25.4 & 29.0 & 34.5 & 29.0 & 26.9 \\
\hline
\end{tabular}


Table 22. Antigua and Barbuda: Operations of the Consolidated Public Sector (Concluded)

(In millions of Eastern Caribbean dollars)

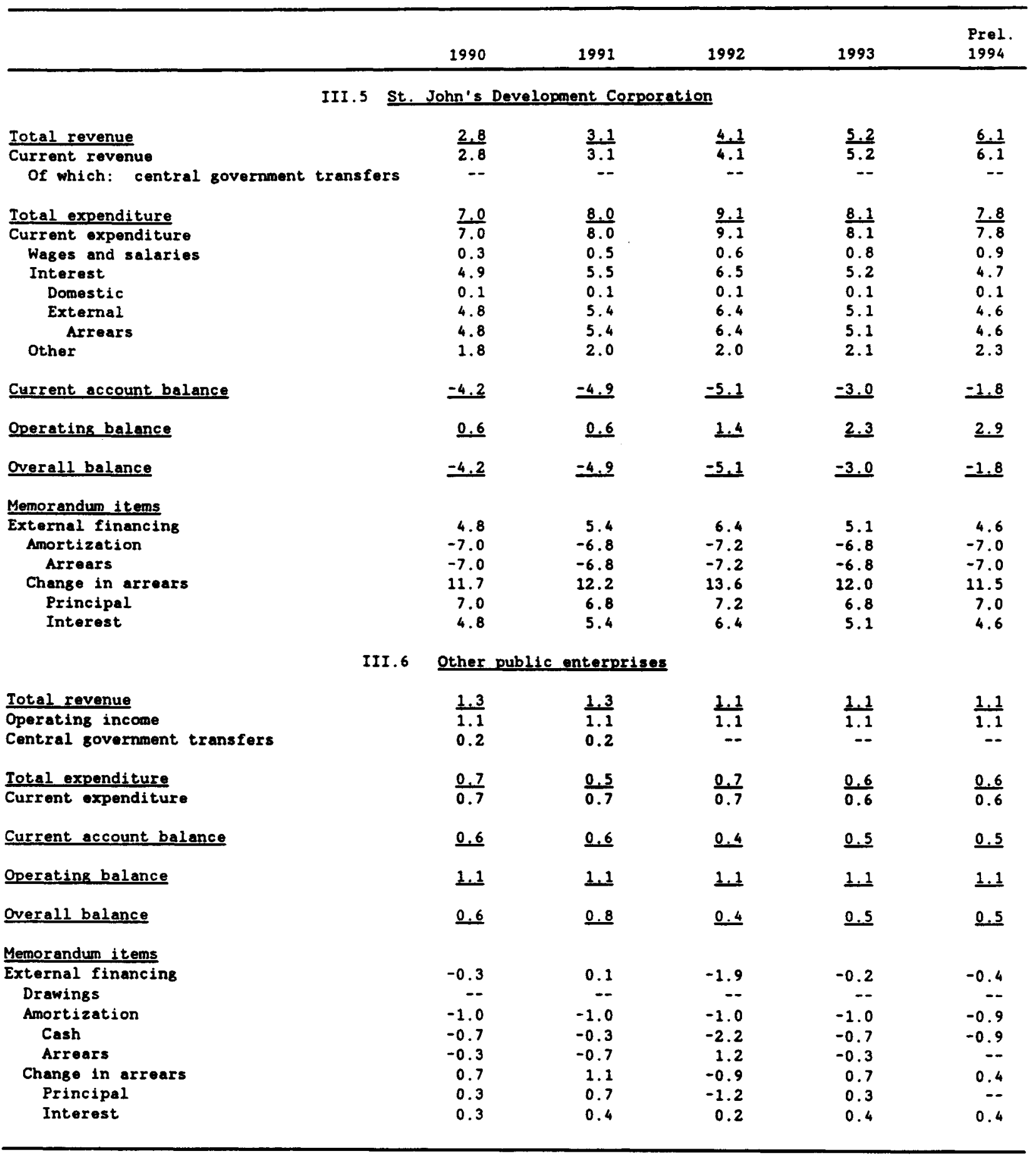

Sources: Ministry of Finance: Social Security Scheme; Medical Benefits Scheme; public enterprises; and Fund staff estimates.

1/ Includes statistical discrepancy between items above and below the line.

2/ Includes write-off of arrears.

3/ Net of rescheduling. 
Table 23. Antigua and Barbuda: Central Government Operations

(In millions of Eastern Caribbean dollars)

\begin{tabular}{|c|c|c|c|c|c|c|c|c|c|c|c|c|c|c|c|}
\hline \multirow[b]{3}{*}{ Current revenue } & \multicolumn{3}{|c|}{1990} & \multicolumn{3}{|c|}{1991} & \multicolumn{3}{|c|}{1992} & \multicolumn{3}{|c|}{1993} & \multicolumn{3}{|c|}{1994 Prel. } \\
\hline & Cash & Arrears & Accrual & Cash & Arrears & Accrual & Cash $A$ & IrrearsA & ccrual & Cash & Arrears & Accrual & Cash & Arrears & Accrual \\
\hline & $\underline{223.5}$ & $=$ & $\underline{223.5}$ & $\underline{221.5}$ & $=$ & $\underline{221.5}$ & $\underline{241.4}$ & $=$ & $\underline{241.4}$ & $\underline{246.8}$ & $=$ & $\underline{246.8}$ & $\underline{276.0}$ & $=$ & $\underline{276.0}$ \\
\hline Current expenditure & 200.8 & $\underline{34.8}$ & 235.6 & 193.9 & 37.2 & 231.1 & 240.9 & $\underline{25.1}$ & 266.0 & $\underline{245.7}$ & $\underline{10.0}$ & $\underline{255.7}$ & $\underline{268.8}$ & 39.6 & 308.4 \\
\hline Wages and salaries 1 / & $\overline{116.1}$ & $\cdots$ & $\overline{116.1}$ & $\overline{123.0}$ & $\overline{--}$ & $\overline{123.0}$ & $\overline{132.3} 2$ & 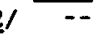 & $\overline{132.3} \underline{2}$ & $\overline{140.5}$ & $\overline{--}$ & $\overline{140.5}$ & $\overline{161.6}$ & -- & $\overline{161.6}$ \\
\hline Established civil servants & 59.3 & -- & 59.3 & 63.8 & $\cdots$ & 63.8 & 67.8 & -- & 67.8 & 71.2 & -- & 71.2 & 83. 3 & -- & 83.3 \\
\hline Nonestablished workers & 56.8 & -- & 56.8 & 59.2 & -- & 59.2 & 64.5 & -- & 64.5 & 69.3 & -- & 69.3 & 78.3 & -. & 78.3 \\
\hline Soclal socurity payments & -- & 5.0 & 5.0 & -- & 5.4 & 5.4 & -- & 5.9 & 5.9 & -- & 6.1 & 6.1 & -- & 7.0 & 7.0 \\
\hline Medical benefit payments & -- & 2.1 & 2.1 & -- & 2.2 & 2.2 & $\cdots$ & 2.8 & 2.8 & -- & 3.0 & 3.0 & -- & 3.3 & 3.3 \\
\hline Other goods and services $\underline{3}$ / & 40.0 & 14.7 & 54.7 & 28.2 & 14.9 & 43.1 & 65.0 & 1.3 & 66.3 & 53.9 & -16.5 & 37.4 & 66.9 & 1.4 & 68.3 \\
\hline Interest payments & 32.9 & 13.0 & 45.9 & 30.7 & 14.7 & 45.4 & 28.5 & 15.1 & 43.6 & 36.8 & 13.4 & 50.2 & 20.8 & 25.9 & 46.6 \\
\hline Domestic & 27.6 & 2.5 & 30.1 & 23.6 & 6.5 & 30.1 & 21.2 & 4.7 & 25.9 & 26.5 & 5.4 & 31.9 & 12.8 & 14.4 & 27.2 \\
\hline External & 5.3 & 10.5 & 15.8 & 7.1 & 8.2 & 15.3 & 7.3 & 10.4 & 17.7 & 10.3 & 8.0 & 18.3 & 7.9 & 11.5 & 19.4 \\
\hline Pensions and gratuities & 9.2 & -- & 9.2 & 10.3 & -- & 10.3 & 12.0 & -- & 12.0 & 10.3 & -- & 10.3 & 14.5 & -- & 14.5 \\
\hline Transfers & 2.6 & -- & 2.6 & 1.8 & -- & 1.8 & 3.1 & -- & 3.1 & 4.2 & 4.0 & 8.2 & 5.0 & 2.0 & 7.0 \\
\hline Private sector & 0.4 & -- & 0.4 & 0.3 & -. & 0.3 & 0.3 & -- & 0.3 & 0.4 & -- & 0.4 & 1.3 & -- & 1.3 \\
\hline International 1 & 1.5 & -- & 1.5 & 0.7 & -- & 0.7 & 1.8 & 1.5 & 3.3 & 2.5 & 4.0 & 6.5 & 2.7 & 2.0 & 4.7 \\
\hline Public sector & 0.7 & -- & 0.7 & 0.8 & -- & 0.8 & 1.0 & -- & 1.0 & 1.3 & -- & 1.3 & 1.0 & -- & 1.0 \\
\hline Current account balance & $\underline{22.7}$ & -34.8 & -12.1 & $\underline{27.6}$ & -37.2 & -9.6 & $\underline{0.5}$ & -25.1 & $\underline{-24.6}$ & $\underline{1.1}$ & -10.0 & -8.9 & $\underline{7.2}$ & -39.6 & -32.4 \\
\hline Capital receipts & $\underline{12.4}$ & $\underline{-}$ & $\frac{12.4}{1.4}$ & 8.0 & $\underline{-}$ & 0.0 & $\frac{11.2}{3.5}$ & $\underline{-}$ & $\frac{11.2}{3.5}$ & $\frac{8.7}{2.1}$ & $=$ & $\frac{8.7}{2.7}$ & $\frac{9.2}{3.0}$ & 二 & $\frac{9.2}{3.0}$ \\
\hline Capital revenue & 7.1 & $\overline{--}$ & 7.1 & $\overline{4.4}$ & -- & $\overline{4.4}$ & $\overline{3.5}$ & $=$ & $\overline{3.5}$ & $\overline{2.4}$ & $=$ & $\overline{2.4}$ & 3.0 & -- & 3.0 \\
\hline Foreign grants & 5.3 & -- & 5.3 & 3.6 & -- & 3.6 & 7.7 & $\cdots$ & 7.7 & 6.3 & -- & 6.3 & 6.2 & - & 6.2 \\
\hline Capital expenditure and net lending & 19.4 & $\underline{-}$ & $\underline{19.4}$ & $\underline{33.1}$ & 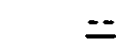 & 33.1 & $\underline{10.7}$ & $=$ & $\underline{10.7}$ & $\underline{26.3}$ & $=$ & $\underline{26.3}$ & $\underline{42.3}$ & 二- & 42.3 \\
\hline Land purchases & $\overline{1.3}$ & $\overline{--}$ & $\overline{1.3}$ & $\overline{--}$ & $=-$ & -- & $\overline{0.4}$ & $=$ & $\overline{0.4}$ & $\overline{3.5}$ & $\overline{-}$ & $\overline{3.5}$ & -- & $\overline{-}$ & $\overline{--}$ \\
\hline Fixed capital expenditure & 15.9 & -- & 15.9 & 33.1 & -- & 33.1 & 10.3 & -- & 10.3 & 22.8 & -- & 22.8 & 32.3 & - & 32.3 \\
\hline Externally financed projects & 15.9 & -- & 15.9 & 26.1 & -- & 26.1 & 8.4 & -- & 8.4 & 6.3 & -- & 6.3 & 11.6 & - & 11.6 \\
\hline Loans & 10.4 & -- & 10.4 & 22.5 & -- & 22.5 & 0.6 & -- & 0.6 & -- & -- & -- & 4.4 & -- & 4.4 \\
\hline Grants & 5.3 & -- & 5.3 & 3.6 & -- & 3.6 & 7.7 & -- & 7.7 & 6.3 & -- & 6.3 & 6.2 & - & 6.2 \\
\hline Local contribution & 0.2 & -- & 0.2 & -- & - & - & 0.1 & -- & 0.1 & -- & - & -- & 1.0 & -- & 1.0 \\
\hline Locally financed projects & -- & -- & -- & 7.0 & -- & 7.0 & 1.9 & -- & 1.9 & 16.5 & - & 16.5 & 20.7 & -- & 20.7 \\
\hline Net lending & -- & - & $\cdots$ & - & -- & -- & -- & -- & -- & -- & - & -- & 10.0 & -- & 10.0 \\
\hline Iransfers to the public sector & 2.2 & $\cdots$ & 2.2 & -- & -- & $\cdots$ & -- & - & -- & - & - & -- & -- & - & -- \\
\hline Overall deficit & $\underline{15.7}$ & -34.8 & -19.1 & $\underline{2.5}$ & -37.2 & -34.7 & $\underline{1.0}$ & -25.2 & -24.1 & -16.5 & -10.0 & $\underline{-26.5}$ & -25.9 & -39.6 & -65.5 \\
\hline
\end{tabular}


Tablo 23. Antigua and Barbuda: Central Government Operations (Concluded)

(In millions of Eastern Caribbean dollars)

\begin{tabular}{|c|c|c|c|c|c|c|c|c|c|c|c|c|c|c|c|}
\hline \multirow[b]{3}{*}{ Financing } & \multicolumn{3}{|c|}{1990} & \multicolumn{3}{|c|}{1991} & \multicolumn{3}{|c|}{1992} & \multicolumn{3}{|c|}{1993} & \multicolumn{3}{|c|}{1994 Prel. } \\
\hline & \multirow{2}{*}{$\begin{array}{c}\text { Cash } \\
-15.7\end{array}$} & Arrears & \multirow{2}{*}{$\begin{array}{r}\text { Accrual } \\
19.1\end{array}$} & \multirow{2}{*}{$\begin{array}{l}\text { Cash } \\
-2.5\end{array}$} & Arrears & \multirow{2}{*}{$\begin{array}{r}\text { Accrual } \\
34,7\end{array}$} & \multicolumn{3}{|c|}{ Cash Arrearsaccrual } & \multirow{2}{*}{$\begin{array}{l}\text { Cash } \\
16.5\end{array}$} & Arrears & \multirow{2}{*}{$\frac{\text { Accrual }}{26.5}$} & \multirow{2}{*}{$\begin{array}{l}\text { Cash } \\
25.9\end{array}$} & Arrears & \multirow{2}{*}{$\begin{array}{r}\text { Accrual } \\
65.5\end{array}$} \\
\hline & & 34.8 & & & $\underline{37.2}$ & & -1.0 & 25.1 & 24.1 & & 10.0 & & & 39.6 & \\
\hline External financing (net) & -11.5 & 31.9 & 20.3 & $\overline{8.6}$ & -8.9 & -0.2 & $-\overline{12.7}$ & -10.9 & -23.6 & -13.9 & -10.1 & -24.0 & -8.8 & -9.9 & $-\overline{18.7}$ \\
\hline Drawings & 10.4 & -- & 10.6 & 22.5 & - & 22.5 & 0.6 & -- & 0.6 & -- & -- & -- & 4.4 & -- & 4.4 \\
\hline Amortization & -21.1 & -5.8 & -27.0 & -13.8 & -8.9 & -22.7 & $-13 \cdot 3$ & -10.9 & -24.1 & -13.9 & -10.1 & -24.0 & -13.2 & -9.9 & -23.1 \\
\hline Rescheduled arrears & -- & 37.7 & 37.7 & -- & -- & -- & -- & -- & -- & -- & -- & -- & -- & -- & -- \\
\hline Change in foretgn assets & -0.8 & -- & -0.8 & -- & -- & -- & -- & -. & -- & -- & -- & -- & -- & -- & -- \\
\hline Domestic Innencing (net) & 0.4 & 12.3 & 12.7 & -11.1 & 5.9 & -5.2 & 11.7 & -- & 11.7 & 30.4 & -. & 30.4 & 28.6 & -- & 28.6 \\
\hline ECCB (not) & 7.2 & -- & 7.2 & 1.5 & -- & 1.5 & -8.6 & -- & -8.6 & 2.3 & -- & 2.3 & 0.1 & -- & 0.1 \\
\hline Comnerclel banks & -6.2 & -- & -6.2 & -6.4 & -- & -6.4 & 15.0 & -- & 15.0 & 21.0 & -- & 21.0 & 6.0 & -- & 6.0 \\
\hline Soctal Security Scheme & 1.3 & 12.3 & 13.6 & -0.7 & 5.9 & 5.2 & -- & -- & -- & -- & -- & -- & -- & -- & -- \\
\hline Rest of public sector & 0.1 & -- & 0.1 & -1.7 & -- & -1.7 & -- & -- & -- & -- & -- & -- & -- & -- & - \\
\hline Private sector bills and bonds & -2.0 & -- & -2.0 & -3.8 & $\cdots$ & -3.8 & 5.3 & -- & 5.3 & 4.9 & -- & 4.9 & 5.0 & -- & 5.0 \\
\hline Other private sector & -- & -- & -- & -- & -- & -- & -- & -- & -- & 2.2 & -- & 2.2 & 17.5 & -- & 17.5 \\
\hline Change in arrears (not) & -4.6 & -9.4 & -14.0 & -- & 40.2 & 40.2 & -- & 35.9 & 35.9 & -- & 20.1 & 20.1 & 6.1 & 49.5 & 55.6 \\
\hline External 4 / & -- & -21.4 & -21.4 & -- & 17.1 & 17.1 & -- & 21.2 & 21.2 & -- & 18.1 & 18.1 & -- & 21.4 & 21.4 \\
\hline Amortization & -- & -18.8 & -18.8 & -- & 8.9 & 8.9 & -- & 10.9 & 10.9 & -- & 10.1 & 10.1 & -- & 9.9 & 9.9 \\
\hline Interest & -- & -2.6 & -2.6 & -- & 8.2 & 8.2 & -- & 10.4 & 10.4 & -- & B. 0 & 8.0 & -- & 11.5 & 11.5 \\
\hline Domestic & -4.6 & 12.0 & 7.4 & - & 23.1 & 23.1 & -- & 14.7 & 14.7 & -- & 2.0 & 2.0 & 6.1 & 28.1 & 34.2 \\
\hline Social Socurity Scheme & -- & -1.8 & -1.8 & -- & 4.9 & 4.9 & -- & 11.2 & 11.2 & -- & 12.1 & 12.1 & -- & 13.5 & 13.5 \\
\hline Medical Benefits Schome & -- & 4.3 & 4.3 & -- & 4.5 & 4.5 & -- & 5.5 & 5.5 & -- & 5.9 & 5.9 & -- & 6.6 & 6.6 \\
\hline Interest (to private sector) & -- & -- & -- & $\cdots$ & 3.9 & 3.9 & -- & 1.6 & 1.6 & -- & 0.4 & 0.4 & -- & 4.3 & 4.3 \\
\hline Floating debt & -4.6 & 9.5 & 4.9 & -- & 9.8 & 9.8 & -- & -3.7 & -3.7 & -- & -5.8 & -5.8 & 6.1 & 1.7 & 7.8 \\
\hline Other & -- & -- & -- & -- & - & -- & -- & 0.1 & 0.1 & -- & -10.6 & -10.6 & -- & 2.0 & 2.0 \\
\hline \multicolumn{16}{|c|}{ (In percent of GDP) } \\
\hline Total revenue and grants & & & 22.1 & & & 20.0 & & & 21.5 & & & 20.8 & & & 21.4 \\
\hline of which: current rovenue & & & 21.0 & & & 19.3 & & & 20.6 & & & 20.1 & & & 20.7 \\
\hline Totel expenditure and net lending & & & 23.9 & & & 23.1 & & & 23.6 & & & 22.9 & & & 26.3 \\
\hline Current expenditure & & & 22.1 & & & 20.2 & & & 22.6 & & & 20.8 & & & 23.2 \\
\hline Wages and solaries 1 & & & 10.9 & & & 10.7 & & & 11.3 & & & 11.4 & & & 12.1 \\
\hline Goods and services $\underline{3}$ / & & & 5.1 & & & 3.8 & & & 5.6 & & & 3.0 & & & 5.1 \\
\hline Other & & & 6.1 & & & 5.7 & & & 5.7 & & & 6.3 & & & 5.9 \\
\hline Capital expenditure and net lending & & & 1.8 & & & 2.9 & & & 0.9 & & & 2.1 & & & 3.2 \\
\hline Central government saving & & & -1.1 & & & -0.8 & & & -2.1 & & & -0.7 & & & -2.4 \\
\hline Overall surplus or deflelt & & & -1.8 & & & -3.0 & & & -2.0 & & & -2.2 & & & -4.9 \\
\hline Change in arrears & & & -1.3 & & & 3.5 & & & 3.1 & & & 1.6 & & & 4.2 \\
\hline External & & & -2.0 & & & 1.5 & & & 1.8 & & & 1.5 & & & 1.6 \\
\hline Domestic & & & 0.7 & & & 2.0 & & & 1.3 & & & 0.2 & & & 2.6 \\
\hline
\end{tabular}

Sources: Ministry of Finance; and Fund staff estimates.

1 ' On a cash basis.

$2 /$ Includes retroactive payment of EC\$6.5 million corresponding to increase granted in 1990.

3 Includes statistical discrepancy between 1 tems above and below the line.

4/ Net of rescheduling. 
Table 24. Antigua and Barbuda: Central Government Revenue and Grants

(In millions of Eastern Caribbean dollars)

\begin{tabular}{|c|c|c|c|c|c|}
\hline & 1990 & 1991 & 1992 & 1993 & $\begin{array}{l}\text { Prel } \\
1994 \\
\end{array}$ \\
\hline Total revenue and grants & $\underline{235.9}$ & $\underline{229.5}$ & $\underline{252.6}$ & $\underline{255.5}$ & $\underline{285.2}$ \\
\hline Current revenue & $\underline{223.5}$ & $\underline{221.5}$ & $\underline{241.4}$ & $\underline{246.8}$ & $\underline{276.0}$ \\
\hline Iax revenue & 194.4 & $\underline{192.9}$ & 201.1 & $\underline{211.4}$ & 236.4 \\
\hline Taxes on income & $\overline{27.5}$ & $\overline{23.7}$ & $\overline{23.5}$ & 23.9 & 25.3 \\
\hline Corporation tax & 26.1 & 22.6 & 22.3 & 22.3 & 23.4 \\
\hline Business tax & 1.2 & 1.0 & 1.2 & 1.3 & 1.6 \\
\hline Incone tex & 0.2 & 0.1 & -- & 0.2 & 0.2 \\
\hline Taxes on property & 3.1 & 3.2 & 2.7 & 3.2 & 2.7 \\
\hline Taxes on domestic production and consumption & 78.5 & 84.5 & 91.0 & 94.8 & 105.2 \\
\hline Consumption duties & 57.5 & 63.6 & 67.5 & 70.9 & 74.4 \\
\hline Sales tax & -- & -- & -- & -- & 1.1 \\
\hline Casino tax & 1.0 & 0.4 & 0.4 & 0.6 & 0.7 \\
\hline Telocommunications tax & 6.4 & 6.5 & 7.6 & 8.0 & 9.1 \\
\hline Stamp duties & 6.1 & 6.6 & 7.3 & 5.9 & 10.1 \\
\hline Business license & -- & -- & -- & -- & -- \\
\hline Other & 7.5 & 7.4 & 8.2 & 9.4 & 9.8 \\
\hline Taxes on international transactions & 85.3 & 81.5 & 83.9 & 89.4 & 103.2 \\
\hline Import duty & 43.8 & 42.4 & 42.5 & 44.0 & 47.0 \\
\hline Customs surcharge & 1.4 & 2.0 & 2.1 & 2.1 & 2.3 \\
\hline Customs service charge & 8.3 & 8.0 & 8.3 & 8.8 & 15.3 \\
\hline Hotel and guest tax & 14.1 & 12.4 & 12.8 & 15.3 & 16.6 \\
\hline Hotel guest lovy & 4.2 & 3.7 & 3.6 & 4.5 & 4.8 \\
\hline Travel tax & 2.0 & 2.0 & 2.3 & 2.1 & 2.7 \\
\hline Embarkation tax & 3.9 & 4.2 & 5.0 & 5.8 & 6.1 \\
\hline Foreign currency levy & 6.3 & 4.8 & 5.2 & 4.7 & 5.3 \\
\hline Cruise-ship passonger tax & 1.3 & 1.7 & 2.1 & 2.0 & 2.9 \\
\hline Other & -- & 0.3 & -- & 0.1 & 0.2 \\
\hline Nontex revenue & 29.1 & 28.6 & 40.3 & 35.4 & 39,7 \\
\hline ECCB profits & 4.3 & $\overline{4.1}$ & 4.1 & $\overline{4.2}$ & $\overline{4.2}$ \\
\hline Lease of bases & 6.9 & 6.9 & 6.9 & 6.9 & 6.9 \\
\hline Licenses and fees & 4.9 & 5.3 & 6.8 & 7.4 & 11.7 \\
\hline Post Office revenue (net) & 0.7 & 0.2 & 0.9 & 1.2 & 0.9 \\
\hline Other & 12.3 & 12.1 & 21.6 & 15.8 & 16.0 \\
\hline $\begin{array}{c}\text { Of which: West Indies Oil Company } \\
\text { profits share }\end{array}$ & -- & -- & 10.8 & 4.9 & 4.2 \\
\hline Capital receipts & $\underline{7.1}$ & $\underline{4.4}$ & $\underline{3.5}$ & $\underline{2.4}$ & $\underline{3.0}$ \\
\hline Land sales & $\overline{6.8}$ & $\overline{4.4}$ & $\overline{3.5}$ & 2.4 & $\overline{3.0}$ \\
\hline Other & 0.3 & -- & - & -- & -- \\
\hline External srants & $\underline{5.3}$ & $\underline{3.6}$ & $\underline{7.7}$ & $\underline{6.3}$ & $\underline{6.2}$ \\
\hline
\end{tabular}

Sources: Ministry of Finance; and Fund staff estimates. 
Table 25. Antigua and Barbuda: Central Government Expenditure

(In millions of Eastern Caribbean dollars)

\begin{tabular}{|c|c|c|c|c|c|}
\hline & 1990 & 1991 & 1992 & 1993 & $\begin{array}{l}\text { Prel. } \\
1994\end{array}$ \\
\hline Total expenditure & $\underline{255.0}$ & $\underline{264.2}$ & $\underline{278.2}$ & $\underline{282.0}$ & $\underline{350.7}$ \\
\hline Current expenditure & $\underline{235.6}$ & 231.1 & 267.5 & 255.7 & 308.4 \\
\hline Wages and salaries 1 & $\overline{116.1}$ & $\overline{123.0}$ & $\overline{132.3} \underline{2}$ & $\overline{140.5}$ & $\overline{161.6}$ \\
\hline $\begin{array}{l}\text { Established civil servants } \\
\text { Nonestablished workers }\end{array}$ & $\begin{array}{l}59.3 \\
56.8\end{array}$ & $\begin{array}{l}63.8 \\
59.2\end{array}$ & $\begin{array}{l}67.8 \\
64.5\end{array}$ & $\begin{array}{l}71.2 \\
69.3\end{array}$ & $\begin{array}{l}83.3 \\
78.3\end{array}$ \\
\hline Social security payments & 5.0 & 5.4 & 5.9 & 6.1 & 7.0 \\
\hline Payments in arrears & 5.0 & 5.4 & 5.9 & 6.1 & 7.0 \\
\hline Medical bonefit payments & 2.1 & 2.2 & 2.8 & 3.0 & 3.3 \\
\hline Payments in arrears & 2.1 & 2.2 & 2.8 & 3.0 & 3.3 \\
\hline Other goods and services $\underline{3} /$ & 54.7 & 43.1 & 66.3 & 37.4 & 68.3 \\
\hline Payments in arrears & 14.7 & 14.9 & 1.3 & -16.5 & 1.4 \\
\hline Interest payments & 45.9 & 45.4 & 43.6 & 50.2 & 46.6 \\
\hline Domestic & 30.1 & 30.1 & 25.9 & 31.9 & 27.2 \\
\hline Cash payments & 27.6 & 23.6 & 21.2 & 26.5 & 12.8 \\
\hline Payments in arrears & 2.5 & 6.5 & 4.7 & 5.4 & 14.4 \\
\hline External & 15.8 & 15.3 & 17.7 & 18.3 & 19.4 \\
\hline Cash payments & 5.3 & 7.1 & 7.3 & 10.3 & 7.9 \\
\hline Payments in arrears & 10.5 & 8.2 & 10.4 & 8.0 & 11.5 \\
\hline Pensions and gratuitios & 9.2 & 10.3 & 12.0 & 10.3 & 14.5 \\
\hline Transfers & 2.6 & 1.8 & 4.6 & 8.2 & 7.0 \\
\hline Private sector & 0.4 & 0.3 & 0.3 & 0.4 & 1.3 \\
\hline International & 1.5 & 0.7 & 3.3 & 6.5 & 4.7 \\
\hline Public sector & 0.7 & 0.8 & 1.0 & 1.3 & 1.0 \\
\hline Capital expenditure and not lending & 19.4 & 33.1 & 10.7 & 26.3 & 42.3 \\
\hline Land purchases & 1.3 & -- & 0.4 & 3.5 & $-\infty$ \\
\hline Fixed cepital expenditure & 15.9 & 33.1 & 10.3 & 22.8 & 32.3 \\
\hline External & 15.7 & 26.1 & 8.3 & 6.3 & 10.6 \\
\hline Local & 0.2 & 7.0 & 2.0 & 16.5 & 21.7 \\
\hline Net lending & -- & -- & -- & -- & 10.0 \\
\hline Transfors to rest of public sector & 2.2 & -- & -- & -- & -- \\
\hline \multicolumn{6}{|l|}{ Memorandum item } \\
\hline Buildup of arrears on current expenditure & 34.8 & 37.2 & 25.1 & 6.0 & 37.6 \\
\hline
\end{tabular}

Sources: Ministry of Finance; and Fund staff estimates.

1/ On a cash basis.

2/ Includes retroactive payment of EC\$6.5 million corresponding to increase granted in 1990.

3/ Includes statistical discrepancy between items above and below the line. 
Table 26. Antigua and Barbuda: Commercial Banks' Operations

(In millions of Eastern Caribbean dollars)

\begin{tabular}{|c|c|c|c|c|c|}
\hline & \multicolumn{5}{|c|}{ December 31} \\
\hline & & & & & Est. \\
\hline & 1990 & 1991 & 1992 & 1993 & 1994 \\
\hline Net foreign assets & -70.9 & -2.0 & -36.1 & $\underline{5.5}$ & 32.4 \\
\hline Foreign assets & $\overline{81.8}$ & $\overline{133.4}$ & $\overline{144.3}$ & $25 \overline{5.9}$ & $\overline{173.1}$ \\
\hline Foreign currency holdings & 4.8 & 4.0 & 6.5 & 5.0 & 6.2 \\
\hline Claims on ECCB area banks & 14.8 & 17.8 & 29.1 & 19.2 & 28.0 \\
\hline Claims on banks abroad & 44.5 & 95.2 & 70.3 & 75.1 & 75.9 \\
\hline Other & 17.6 & 16.4 & 38.4 & 56.6 & 63.0 \\
\hline Foreign liabilities & -152.7 & -135.4 & -180.3 & -150.4 & -140.7 \\
\hline Balances due to ECCB area banks & -31.6 & -26.9 & -43.1 & -24.3 & -22.9 \\
\hline Balances due to banks abroad & -59.4 & -50.0 & -66.8 & -56.5 & -50.7 \\
\hline Nonresident deposits & -61.0 & -58.1 & -69.2 & -68.5 & -66.8 \\
\hline Demand & -10.6 & -4.2 & -13.3 & -9.1 & -7.4 \\
\hline Savings & -20.0 & -19.6 & -24.5 & -27.9 & -28.2 \\
\hline Time & -28.3 & -31.6 & -30.6 & -28.6 & -26.9 \\
\hline Foreign currency & -2.0 & -2.7 & -0.8 & -2.9 & -4.3 \\
\hline Other & -0.8 & -0.4 & -1.2 & -1.2 & -0.4 \\
\hline Net position with ECCB & $\underline{62.2}$ & 77.1 & 97.8 & 91.1 & 79.0 \\
\hline Claims on ECCB & $\overline{63.3}$ & $\overline{77.2}$ & $\overline{98.3}$ & 91.7 & 79.0 \\
\hline Currency holdings & 11.6 & 14.3 & 9.7 & 13.5 & 10.3 \\
\hline Current deposits & 10.2 & 14.8 & 38.3 & 24.2 & 7.6 \\
\hline Fixed deposits & 4.0 & 6.0 & 6.0 & 3.3 & 6.0 \\
\hline Statutory reserves & 37.0 & 42.1 & 44.4 & 50.7 & 55.1 \\
\hline Statutory deposits & 0.6 & -- & -- & -- & -- \\
\hline Liabilities to ECCB & -1.1 & -0.1 & -0.5 & -0.5 & -- \\
\hline Net domestic essets & 513.0 & $\underline{512.4}$ & 543.8 & 591.0 & 638.2 \\
\hline Net credit to Govermment & 79.8 & 73.4 & 88.4 & 109.4 & 113.0 \\
\hline Treasury bills & 9.7 & 8.1 & 23.1 & 24.1 & 22.2 \\
\hline Debentures & 14.9 & 14.4 & 14.4 & 14.4 & 13.9 \\
\hline Loans and advances & 59.1 & 57.6 & 58.9 & 80.7 & 84.3 \\
\hline Deposits & -3.9 & -6.7 & -7.9 & -9.7 & -7.3 \\
\hline Net credit to statutory bodies & -24.6 & -34.2 & -42.5 & -41.4 & -37.2 \\
\hline Loans and advances & 8.4 & 6.3 & 11.8 & 9.7 & 14.0 \\
\hline Deposits & -33.0 & -40.4 & -54.4 & -51.1 & -51.2 \\
\hline Net credit to other & & & & & \\
\hline Einancial institutions & 1.8 & -3.1 & -35.8 & -30.8 & -28.5 \\
\hline Loans, advances, and investments & 22.3 & 23.2 & 3.5 & 5.9 & 6.4 \\
\hline Deposits & -20.5 & -26.3 & -39.3 & -36.7 & -34.9 \\
\hline Credit to private sector & 503.1 & 547.4 & 586.7 & 625.3 & 650.3 \\
\hline Interbank float & -1.1 & 6.5 & 9.9 & 1.3 & 1.2 \\
\hline Claims & 4.7 & 8.4 & 12.3 & 5.8 & 12.4 \\
\hline Liabilities & -5.8 & -1.9 & -2.4 & -4.5 & -11.2 \\
\hline Net unclessified assets & -45.9 & -77.6 & -62.9 & -72.8 & -60.7 \\
\hline Assets & 55.6 & 52.5 & 53.5 & 63.8 & 85.6 \\
\hline Liabilities & -101.5 & -130.2 & -116.4 & -136.6 & -146.3 \\
\hline Liabilities to the private sector & $\underline{504.3}$ & 587.5 & 605.5 & 687.7 & 749.6 \\
\hline Demand deposits & 86.4 & 97.0 & $\overline{104.1}$ & $\overline{113.8}$ & $\overline{123.5}$ \\
\hline Savings deposits & 188.1 & 203.6 & 224.1 & 282.9 & 320.0 \\
\hline Time deposits & 207.6 & 254.6 & 244.2 & 265.9 & 274.4 \\
\hline Foreign currency deposits & 22.2 & 32.4 & 33.1 & 25.1 & 31.7 \\
\hline
\end{tabular}

Sources: Eastern Caribbean Central Bank; and comercial banks. 
Table 27. Antigua and Barbuda: Distribution of Commercial Bank Credit to Private Sector

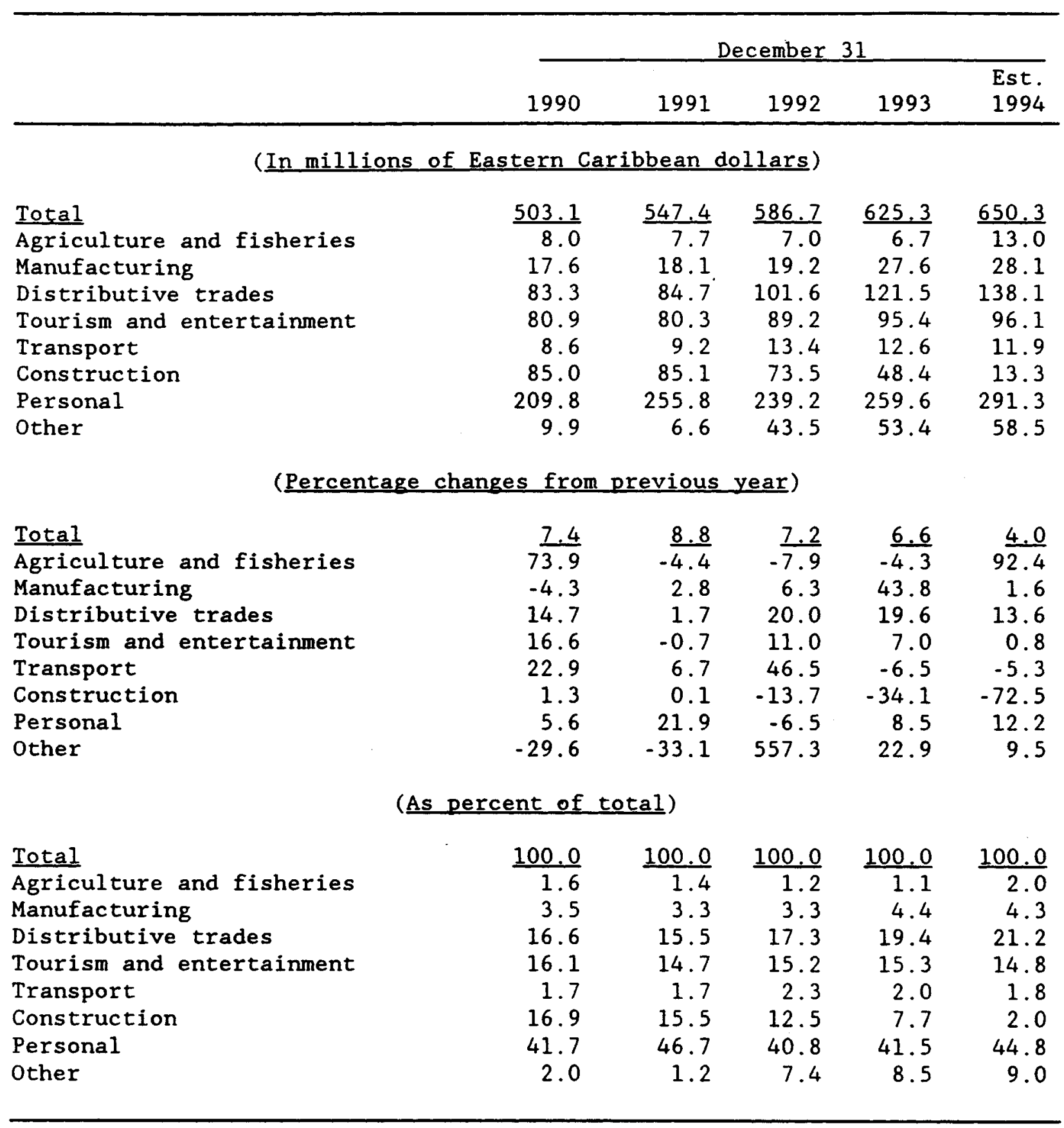

Source: Eastern Caribbean Central Bank. 
Table 28. Antigua and Barbuda: Selected Interest Rates

(In percent per annum at end of period)

\begin{tabular}{llllll}
\hline & & & & Est. \\
& 1990 & 1991 & 1992 & 1993 & 1994 \\
\hline
\end{tabular}

I. Commercial Bank Rates 1/

$\begin{array}{lrrrrr}\begin{array}{l}\text { Deposits } \\ \text { Savings } \\ \text { Time }\end{array} & 4.0-7.5 & 4.0-7.5 & 4.0-8.0 & 4.0-8.0 & 4.0-8.0 \\ \quad \begin{array}{l}\text { 3-month } \\ \text { - -month } \\ \text { 12-month }\end{array} & 4.0-8.5 & 4.0-8.0 & 2.0-7.0 & 2.0-6.5 & 2.0-6.5 \\ & 4.0-9.0 & 4.0-8.0 & 2.0-7.5 & 2.0-7.0 & 2.0-7.0 \\ \text { Lending } & 4.0-10.0 & 4.0-8.0 & 2.0-8.0 & 2.0-7.5 & 2.0-8.0 \\ \begin{array}{l}\text { Prime } \\ \text { Other }\end{array} & & & & & \\ & 10.5-12.0 & 11.0-13.0 & 11.0-13.0 & 10.5-13.0 & 10.0-12.0 \\ & 10.5-23.2 & 11.0-23.2 & 11.0-24.4 & 10.5-24.0 & 8.5-24.0\end{array}$

II. Other Rates

Government

Treasury bill rate $\underline{2} /$

6.5

7.5

7.7

7.7

7.7

7.7

Debenture rate

9.0

9.0

9.0

9.0

$\underline{E C C B}$

Bankers' deposits rate $\underline{3} /$

Lending rate to banks
$6.75-8.0 \quad 4.40-4.67$
10.5
10.0

$2.84-3.48$

$2.72-3.54$

$5.79-7.46$

10.5

10.5

Sources: Eastern Caribbean Central Bank; commercial banks; and Ministry of Finance.

1/ Ranges show variations among banks.

2/ Issue rate; from 1991, rates are weighted averages.

3/ Rates vary with the term of deposits. 
Table 29. Antigua and Barbuda: Balance of Payments

(In millions of U.S. dollars)

\begin{tabular}{|c|c|c|c|c|c|c|}
\hline & \multicolumn{3}{|c|}{1990} & \multicolumn{3}{|c|}{1991} \\
\hline & Credit & Debit & Net & Credit & Debit & $\overline{\text { Net }}$ \\
\hline Goods, services, and transfers & 419.1 & 477.1 & -58.1 & $\underline{454.3}$ & 494.1 & -39.7 \\
\hline Merchandise & $\underline{33.4}$ & $\underline{358.8}$ & -325.4 & 49.5 & 375.9 & -326.4 \\
\hline$\frac{\text { Services }}{\text { Travel }}$ & $\frac{371.2}{298.3}$ & $\frac{113.2}{18.0}$ & $\frac{258.0}{280.3}$ & $\frac{389.0}{308.9}$ & $\frac{112.1}{20.4}$ & $\frac{276.9}{288.5}$ \\
\hline Other nonfactor services & 70.4 & 45.6 & 24.8 & 77.4 & 50.3 & 27.1 \\
\hline Rassenger services & 41.9 & 9.7 & 32.2 & 55.3 & 10.9 & 44.4 \\
\hline Port services & 4.8 & 5.4 & -0.6 & 7.4 & 4.6 & 2.8 \\
\hline Other goods and services & 23.7 & 21.3 & 2.4 & 14.7 & 24.2 & -9.5 \\
\hline official & 2.6 & 3.7 & -1.1 & 2.6 & 3.7 & -1.1 \\
\hline Private & 21.1 & 17.7 & 3.4 & 12.2 & 20.6 & -8.4 \\
\hline Insurance $\underline{2}$ & 11.8 & 12.3 & -0.5 & 3.8 & 10.9 & -7.2 \\
\hline Other & 9.3 & 5.4 & 3.9 & 8.4 & 9.7 & -1.2 \\
\hline Services on major projects $\underline{3}$ / & -- & 9.1 & -9.1 & -- & 10.6 & -10.6 \\
\hline Public sector interest $1 /$ & -- & 26.9 & -26.9 & -- & 25.7 & -25.7 \\
\hline Due and paid & 0.5 & 3.0 & -2.5 & -- & 2.9 & -2.9 \\
\hline Repayment in arrears & -0.5 & 23.8 & -24.3 & -- & 22.8 & -22.8 \\
\hline Commercial banks' interest & 0.5 & 0.9 & -0.4 & 1.1 & 1.0 & 0.2 \\
\hline Profit remittances & $\cdots$ & 17.3 & -17.3 & -- & 13.3 & -13.3 \\
\hline Other factor payments 2 / & 2.0 & 4.5 & -2.5 & 1.5 & 1.5 & -- \\
\hline Iransfers & 14.5 & $\underline{5.2}$ & 9.3 & $\underline{15.9}$ & 6.1 & 9.7 \\
\hline Private & 12.5 & 4.6 & $\overline{7.9}$ & $\overline{12.2}$ & $\overline{5.6}$ & 6.6 \\
\hline official & 2.0 & 0.5 & 1.5 & 3.7 & 0.6 & 3.1 \\
\hline Capital account & 45.7 & 38.2 & 2.4 & 52.8 & $\underline{56.0}$ & -3.2 \\
\hline Official drawings on loans & 3.9 & $\cdots$ & 3.9 & $\overline{8.3}$ & - & $\overline{8.3}$ \\
\hline Official obligations on loans & - & 35.7 & -35.7 & -- & 30.5 & -30.5 \\
\hline Repayment made & -- & 8.8 & -8.8 & -- & 5.2 & -5.2 \\
\hline Repayments in arrears & -- & 26.8 & -26.8 & -- & 25.3 & -25.3 \\
\hline Commercial banks & -- & 2.6 & -2.6 & -- & 25.5 & -25.5 \\
\hline Private direct investment & 41.8 & -- & 41.8 & 44.5 & - & 44.5 \\
\hline Qverall balance & & & -50.7 & & & -42.9 \\
\hline Overall financing & $\underline{72.8}$ & $\underline{21,9}$ & 50.7 & 48.0 & $\underline{5.1}$ & 42.9 \\
\hline Change in government foreign assets & -- & 0.3 & $\overline{-0.3}$ & - & $\overline{-\cdots}$ & -- \\
\hline Change in imputed reserves & 0.3 & -- & 0.3 & -- & 5.1 & -5.1 \\
\hline Accumulation of arrears & 50.7 & $\cdots$ & 50.7 & 48.0 & -- & 48.0 \\
\hline Reduction in arrears & -- & 21.9 & -21.9 & -- & -- & -- \\
\hline Rescheduling of arrears/write-offs & 21.9 & - & 21.9 & -- & -- & -- \\
\hline
\end{tabular}


Table 29. Antigua and Barbuda: Balance of Payments (Concluded)

(In millions of U.S, dollars)

\begin{tabular}{|c|c|c|c|c|c|c|c|c|c|}
\hline & \multicolumn{3}{|c|}{1992} & \multicolumn{3}{|c|}{1993} & \multicolumn{3}{|c|}{1994} \\
\hline & Credit & Debit & Net & Credit & Dobit & Net & Credit & Debit & Net \\
\hline Goods, services, and transfers & $\underline{497.3}$ & $\underline{531.0}$ & -33.7 & $\underline{526.0}$ & 557.4 & $\underline{-31.3}$ & $\underline{557.8}$ & $\underline{584.4}$ & -26.7 \\
\hline Merchandise & $\underline{69.6}$ & 417.0 & -347.4 & $\underline{50.9}$ & $\underline{425.8}$ & -374.9 & 40.9 & 443.8 & -402.9 \\
\hline Services & $\frac{414.0}{2.10}$ & $\underline{108.1}$ & 305,9 & $\frac{460.0}{1.0}$ & 124.8 & 335,2 & 502.9 & $\underline{133.3}$ & 369.6 \\
\hline Travel & 331.9 & 22.6 & 309.4 & 376.4 & 23.4 & 353.0 & 415.2 & 24.6 & 390.6 \\
\hline Other nonfactor services & 78.7 & 39.0 & 39.7 & 80.9 & 59.8 & 21.1 & 84.9 & 63.5 & 21.4 \\
\hline Passenger services & 53.1 & 9.4 & 43.6 & 52.0 & 11.4 & 40.6 & 54.6 & 12.0 & 42.6 \\
\hline Port services & 9.4 & 4.4 & 5.0 & 12.5 & 5.2 & 7.3 & 13.1 & 5.4 & 7.7 \\
\hline Other soods and services & 16.3 & 22.3 & -6.0 & 16.4 & 38.4 & -22.0 & 17.2 & 43.0 & -25.8 \\
\hline Official & 2.6 & 3.7 & -1.1 & 2.6 & 3.7 & -1.1 & 2.6 & 3.7 & -1.1 \\
\hline Private & 13.7 & 18.7 & -4.9 & 13.8 & 34.7 & -20.9 & 14.6 & 39.3 & -24.7 \\
\hline Insurance & 6.0 & 10.9 & -4.9 & 5.5 & 18.6 & -13.1 & 5.8 & 20.0 & -14.2 \\
\hline Other & 7.8 & 7.8 & -- & 8.3 & 16.1 & -7.8 & 8.8 & 19.3 & -10.5 \\
\hline Services on major projects & -- & 2.8 & -2.8 & -- & 4.8 & -4.8 & -- & 3.1 & -3.1 \\
\hline Public sector interest $\underline{1}$ & -- & 29.1 & -29.1 & -- & 27.0 & -27.0 & -- & 27.2 & -27.2 \\
\hline Due and paid & -- & 3.0 & -3.0 & -- & 4.0 & -4.0 & -- & 3.2 & -3.2 \\
\hline Repayment in arrears & -- & 26.1 & -26.1 & -- & 23.0 & -23.0 & -- & 24.0 & -24.0 \\
\hline Commercial banks interest & 1.2 & 0.9 & 0.3 & 1.1 & -- & 1.1 & 1.2 & -- & 1.2 \\
\hline Profit remittances & -- & 13.2 & -13.2 & -- & 11.0 & -11.0 & -- & 14.0 & -14.0 \\
\hline Other factor payments & 2.2 & 3.3 & -1.1 & 1.6 & 3.5 & -1.9 & 1.6 & 4.0 & -2.4 \\
\hline Iransfers & $\underline{13.7}$ & 5.9 & 2.8 & $\underline{15.1}$ & 6.8 & 8.3 & 14.0 & 2.3 & 6.8 \\
\hline Private & 10.8 & 5.9 & 4.9 & 11.6 & 6.1 & 5.6 & 11.7 & 6.1 & 5.6 \\
\hline Official & 2.9 & - & 2.9 & 3.5 & 0.8 & 2.7 & 2.3 & 1.1 & 1.2 \\
\hline Capital account 2/ & $\underline{26.7}$ & $\underline{31.7}$ & -5.1 & $\underline{24.1}$ & $\underline{38.3}$ & -14.2 & $\underline{5.5}$ & $\underline{16.3}$ & -10.8 \\
\hline Official drawings on loans & 0.2 & -- & 0.2 & -- & -- & $\cdots$ & 1.6 & -- & 1.6 \\
\hline Official obligations on loans & -- & 31.7 & -31.7 & - & 22.9 & -22.9 & -- & 16.3 & -16.3 \\
\hline Repayments made & -- & 5.8 & -5.8 & -- & 5.5 & -5.5 & -- & 5.3 & -5.3 \\
\hline Repayments in arrears & -- & 25.9 & -25.9 & -- & 17.4 & -17.4 & -- & 11.0 & -11.0 \\
\hline Conmercial banks & 12.6 & -- & 12.6 & -- & 15.4 & -15.4 & -- & 10.0 & -10.0 \\
\hline Private direct investment & 13.8 & -- & 13.8 & 24.1 & -- & 24.1 & 13.9 & -- & 13.9 \\
\hline Overall balance & & & -38.7 & & & -45.5 & & & -37.4 \\
\hline Overail financing & $\underline{52.0}$ & 13.2 & $\underline{38.7}$ & 40.4 & -5.1 & 45.5 & 35.1 & -2.4 & $\underline{37.4}$ \\
\hline Change in government foreign assets & -- & -- & -- & -- & -- & -- & -- & -- & -- \\
\hline Change in imputed reserves & $\cdots$ & 13.2 & -13.2 & -- & -5.1 & 5.1 & - & -2.4 & 2.4 \\
\hline Accumulation of arrears & 52.0 & -- & 52.0 & 40.4 & -- & 40.4 & 35.1 & -- & 35.1 \\
\hline Reduction in arrears & -- & -- & - & -- & -- & -- & -- & -- & - \\
\hline Rescheduling of arrears/write-offs & -- & -- & -- & -- & -- & $\cdots$ & $\cdots$ & -- & -- \\
\hline
\end{tabular}

Sources: Statistics Division. Ministry of Finance; ECCB; Department of Tourism; and Fund staff estimates.

1/ Data cover only official external debt because of lack of information on private debt.

2/ Exsludes capital flows to or from the nonbank domestic private sector because of lack of information. 
Table 30. Antigua and Barbuda: Selected Tourism Statistics

\begin{tabular}{|c|c|c|c|c|c|}
\hline & 1990 & 1991 & 1992 & 1993 & $\begin{array}{l}\text { Prel } \\
1994\end{array}$ \\
\hline \multicolumn{6}{|c|}{ (Number of visitors) } \\
\hline Total visitors & 457,512 & 478,958 & 485,135 & $\underline{498,839}$ & 517,839 \\
\hline Cruise-ship & 236,007 & 266,524 & 258,827 & $\overline{246,609}$ & 251,213 \\
\hline Yacht & 24,459 & 15,863 & 16,406 & 15,500 & 16,275 \\
\hline Air stayovers & 184,248 & 182,188 & 193,589 & 221,230 & 234,541 \\
\hline Private homes and others & 12,798 & 14,383 & 16,313 & 15,500 & 15,810 \\
\hline \multicolumn{6}{|c|}{ (Share in total number of visitors) } \\
\hline Total visitors & 100.0 & 100.0 & $\underline{100.0}$ & $\underline{100.0}$ & 100.0 \\
\hline Cruise-ship & 51.6 & 55.6 & 53.4 & 49.4 & 48.5 \\
\hline Yacht & 5.3 & 3.3 & 3.4 & 3.1 & 3.1 \\
\hline Air stayovers & 40.3 & 38.1 & 39.8 & 44.4 & 45.3 \\
\hline Private homes and others & 2.8 & 3.0 & 3.4 & 3.1 & 3.1 \\
\hline \multicolumn{6}{|c|}{ (Annual percentage change) } \\
\hline Total visitors & 8.9 & 4.7 & 1.3 & 2.8 & 3.8 \\
\hline Cruise-ship & 9.0 & $1 \overline{2.9}$ & -2.9 & $-\overline{4.7}$ & $\overline{1.9}$ \\
\hline Yacht & 68.2 & -35.1 & 3.4 & -5.5 & 5.0 \\
\hline Air stayovers & 5.0 & -1.1 & 6.3 & 14.3 & 6.0 \\
\hline Private homes and others & -5.8 & 12.4 & 13.4 & -5.0 & 2.0 \\
\hline \multicolumn{6}{|c|}{ (In number of days) } \\
\hline Average length of stay & $\underline{4.9}$ & 4.7 & 4.9 & 5.3 & $\underline{5.6}$ \\
\hline Cruise-ship & 1.0 & 1.0 & 1.0 & 1.0 & $\overline{1.0}$ \\
\hline Yacht & 10.0 & 10.0 & 10.0 & 10.0 & 10.0 \\
\hline Air stayovers & 8.6 & 8.9 & 9.0 & 9.2 & 9.6 \\
\hline Private homes and others & 14.0 & 14.0 & 14.0 & 14.0 & 14.0 \\
\hline \multicolumn{6}{|c|}{ (In U.S. dollars) } \\
\hline Averase daily expenditure $1 /$ & 86.4 & 84.9 & 88.2 & 93.5 & 94.7 \\
\hline Cruise-ship & 23.4 & 24.8 & 25.8 & 25.8 & $\overline{25.3}$ \\
\hline Yacht & 46.2 & 49.0 & 51.0 & 53.0 & 54.1 \\
\hline Air stayovers & 175.3 & 178.8 & 178.8 & 175.2 & 175.2 \\
\hline Private homes and others & 43.8 & 44.7 & 44.7 & 43.8 & 43.8 \\
\hline \multicolumn{6}{|c|}{ (Annual percentage change) } \\
\hline Average daily expenditure 1/ & 2.0 & $=1.8$ & $\underline{3.9}$ & $\underline{6.1}$ & 1.2 \\
\hline Cruise-ship & 6.0 & 6.0 & $\overline{4.0}$ & $\overline{--}$ & $-\overline{2.0}$ \\
\hline Yacht & 5.0 & 6.0 & 4.0 & 4.0 & 2.0 \\
\hline Air stayovers & 4.0 & 2.0 & -- & -2.0 & -- \\
\hline Private homes and others & 4.0 & 2.0 & -- & -2.0 & - \\
\hline \multicolumn{6}{|c|}{ (In millions of U.S. dollars) } \\
\hline Total expenditure & $\underline{298.3}$ & 308.9 & $\underline{331.9}$ & $\underline{376.4}$ & 415.2 \\
\hline Cruise-ship & 5.5 & 6.6 & 6.7 & 6.4 & 6.3 \\
\hline Yacht & 11.3 & 7.8 & 8.4 & 8.2 & 8.8 \\
\hline Air stayovers & 276.6 & 288.8 & 310.4 & 355.6 & 393.5 \\
\hline Private homes and others & 4.8 & 5.7 & 6.5 & 6.2 & 6.6 \\
\hline \multicolumn{6}{|c|}{ (Annual percentage change) } \\
\hline Total expenditure & 11.7 & $\underline{3.6}$ & 7.5 & 13.4 & $\underline{10.3}$ \\
\hline Cruise-ship & 15.5 & 19.7 & 1.0 & -4.7 & -0.2 \\
\hline Yacht & 76.6 & -31.3 & 7.6 & -1.7 & 7.1 \\
\hline Air stayovers & 10.2 & 4.4 & 7.5 & 14.6 & 10.7 \\
\hline Private homes and others & -1.0 & 18.7 & 14.7 & -4.7 & 6.5 \\
\hline
\end{tabular}

Sources: Statistics Division, Ministry of Finance; Department of Tourism; and Fund staff estimates.

1' Based on an index of room rates computed by Fund staff from rate schedules published by the Antigua and Barbuda Hotels and Tourist Association. 
Table 31. Antigua and Barbuda: Air Stayover Arrivals

\begin{tabular}{|c|c|c|c|c|c|c|}
\hline & 1989 & 1990 & 1991 & 1992 & 2993 & $\begin{array}{l}\text { Prel } \\
1994\end{array}$ \\
\hline \multicolumn{7}{|c|}{ (Number of stayover arrivals) } \\
\hline Total & $\underline{175,500}$ & 184.248 & $\underline{182,188}$ & $\underline{193,589}$ & $\underline{221,230}$ & 234,541 \\
\hline$\overline{J a n u a r y}$ & 17,720 & 16,269 & 14,726 & 16.068 & 19,689 & $21, .135$ \\
\hline February & 18,811 & 17,760 & 15,295 & $18,0 \pm \varepsilon$ & 8,583 & 20,024 \\
\hline March & 17,972 & 16,679 & 15,776 & 16,007 & $\pm 8,583$ & 21,647 \\
\hline April & 16,629 & 19,939 & 18,803 & 17.632 & 22,123 & 23,357 \\
\hline May & 13,270 & 14,096 & 15,011 & 15,534 & 18,805 & 18,911 \\
\hline June & 10,507 & 12,508 & 12,376 & $13, \geq \pm 2$ & $14,60 ?$ & 16,085 \\
\hline July & 14,988 & 14,995 & 16,268 & 17.340 & 19,011 & 21,953 \\
\hline August & 14,515 & 15,343 & 15,780 & 15,882 & 17,920 & 18,614 \\
\hline September & 8,307 & 10,763 & 10,446 & 12,280 & 14,822 & 16,135 \\
\hline October & 10,933 & 12,414 & 13,006 & 14,910 & 18,038 & 17,220 \\
\hline November & 14,747 & 16,334 & 16,652 & 18,077 & 18,366 & 18,480 \\
\hline December & 17,101 & 17,148 & 18,049 & 18,729 & 19,789 & 20,980 \\
\hline \multicolumn{7}{|c|}{ (Annual percentage change) } \\
\hline Total & -0.8 & $\underline{5.0}$ & -1.1 & $\underline{6.3}$ & 14.3 & $\underline{6.0}$ \\
\hline
\end{tabular}

Sources: Statistics Division, Ministry of Finance; and Department of Tourism. 
Table 32. Antigua and Barbuda: Air Stayover Arrivals by Origin

\begin{tabular}{|c|c|c|c|c|c|}
\hline & 1990 & 1991 & 1992 & 1993 & $\begin{array}{l}\text { Prel. } \\
1994\end{array}$ \\
\hline \multicolumn{6}{|c|}{ (In number of arrivals) } \\
\hline $\begin{array}{l}\text { Total stayover arrivals } \\
\text { United States 1/ } \\
\text { Canada } \\
\text { Europe } \\
\text { Caribbean countries } \\
\text { Others }\end{array}$ & $\begin{array}{r}184,248 \\
80,812 \\
15,175 \\
50,592 \\
31,338 \\
6,331\end{array}$ & $\begin{array}{r}182,188 \\
73,865 \\
12,100 \\
56,300 \\
34,122 \\
5,801\end{array}$ & $\begin{array}{r}193,589 \\
75,078 \\
15,483 \\
62,716 \\
36,127 \\
4,185\end{array}$ & $\begin{array}{r}221,230 \\
85,818 \\
15,873 \\
78,497 \\
36,974 \\
4,068\end{array}$ & $\begin{array}{r}\frac{234,541}{82,936} \\
16,424 \\
95,940 \\
31,886 \\
7,355\end{array}$ \\
\hline \multicolumn{6}{|c|}{ (In percent of total arrivals by air) } \\
\hline $\begin{array}{l}\text { United States } 1 / \\
\text { Canada } \\
\text { Europe } \\
\text { Caribbean countries } \\
\text { Others }\end{array}$ & $\begin{array}{r}43.9 \\
8.2 \\
27.5 \\
17.0 \\
3.4\end{array}$ & $\begin{array}{r}40.5 \\
6.6 \\
30.9 \\
18.7 \\
3.2\end{array}$ & $\begin{array}{r}38.8 \\
8.0 \\
32.4 \\
18.7 \\
2.2\end{array}$ & $\begin{array}{r}38.8 \\
7.2 \\
35.5 \\
16.7 \\
1.8\end{array}$ & $\begin{array}{r}35.4 \\
7.0 \\
40.9 \\
13.6 \\
3.1\end{array}$ \\
\hline $\begin{array}{l}\text { Average length of stay } \\
\text { (days) } 2 /\end{array}$ & 8.6 & 8.9 & 9.0 & 9.2 & 9.6 \\
\hline
\end{tabular}

Sources: Tourism Board; and Caribbean Tourism Research and Development Centre.

1/ Includes Puerto Rico and the U.S. Virgin Islands.

2/ Assumed to be 14 days for European, 6 days for U.S. and Canadian, and 7.8 days for all other visitors. 
Table 33. Antigua and Barbuda: Tourism Capacity

\begin{tabular}{|c|c|c|c|c|c|}
\hline & 1990 & 1991 & 1992 & 1993 & $\begin{array}{l}\text { Prel. } \\
1994\end{array}$ \\
\hline \multicolumn{6}{|l|}{ Hoce $1 \mathrm{~s}$} \\
\hline Number of hotels & 53 & 56 & 56 & 56 & 56 \\
\hline Number o $=$ rooms & 2,731 & 2,997 & 2,997 & 3,140 & 3,213 \\
\hline Number of beds & 5,527 & 6,065 & 6,065 & 6,355 & 6,502 \\
\hline \multicolumn{6}{|l|}{ Guest houses } \\
\hline Number of guest houses & 15 & 15 & 15 & 15 & 15 \\
\hline Number of rooms & 64 & 64 & 64 & 64 & 64 \\
\hline Number of beds & 120 & 120 & 120 & 120 & 120 \\
\hline \multicolumn{6}{|l|}{ Apartments } \\
\hline Number of apartments & 36 & 32 & 32 & 32 & 32 \\
\hline Number of rooms & 320 & 256 & 256 & 256 & 256 \\
\hline Number of beds & 650 & 520 & 520 & 520 & 520 \\
\hline \multicolumn{6}{|l|}{ Total } \\
\hline \multicolumn{6}{|l|}{$\overline{\text { Number }}$ of hotels, guest } \\
\hline Number of rooms & 3,115 & 3,317 & 3,317 & 3,460 & 3,533 \\
\hline Number of beds & 6,297 & 6,705 & 6,705 & 6,995 & 7,142 \\
\hline \multicolumn{6}{|l|}{ Estimated occupancy } \\
\hline rate (percent) $1 /$ & $\underline{29.6}$ & $\underline{27.5}$ & $\underline{29.2}$ & $\underline{32.0}$ & 33.2 \\
\hline \multicolumn{6}{|l|}{ Annual change } \\
\hline \multicolumn{6}{|l|}{ Number of hotels, guest } \\
\hline houses, and apartments & -4 & -1 & - - & - & - \\
\hline Number of rooms & 60 & 202 & - & 143 & 73 \\
\hline Number of beds & 37 & 408 & - & 289 & 148 \\
\hline
\end{tabular}

Sources: Statistics Division, Ministry of Finance; Department of Tourism; and Tourism Planning Advisor (under the Commonwealth Fund for Technical Cooperation).

1) Estimated on the basis of the number of arrivals by air and the number of rooms. 
Table 34. Antigua and Barbuda: Visitor Arrivals as a Share of the Caribbean Market

\begin{tabular}{|c|c|c|c|c|c|}
\hline & 1990 & 1991 & 1992 & 1993 & $\begin{array}{l}\text { Prel. } \\
1994\end{array}$ \\
\hline \multicolumn{6}{|c|}{ (In thousands) } \\
\hline \multicolumn{6}{|c|}{ Stayover passenger arrivals $1 /$} \\
\hline Antigua & 221.5 & 212.4 & 226.3 & 252.2 & 266.6 \\
\hline $\begin{array}{l}\text { Caribbean area } \\
\text { Percentage share }\end{array}$ & $11,853.0$ & $11,765.0$ & $12,123.0$ & $12,729.2$ & $13,238.3$ \\
\hline of Antigua & 1.87 & 1.81 & 1.87 & 1.98 & 2.01 \\
\hline \multicolumn{6}{|c|}{$\frac{\text { Cruise-ship passenger }}{\text { arrivals }}$} \\
\hline Antigua & 236.0 & 266.5 & 258.8 & 246.6 & 251.2 \\
\hline $\begin{array}{l}\text { Caribbean area } \\
\text { Percentage share }\end{array}$ & $7,270.0$ & $8,190.0$ & $8,780.0$ & $9,131.2$ & $9,039.9$ \\
\hline of Antigua & 3.25 & 3.25 & 2.95 & 2.70 & 2.78 \\
\hline \multicolumn{6}{|c|}{ (Annual percentage change) } \\
\hline \multicolumn{6}{|c|}{ Stayover passenger arrivals I/ } \\
\hline $\begin{array}{l}\text { Antigua } \\
\text { Caribbean area }\end{array}$ & $\begin{array}{r}8.8 \\
11.1\end{array}$ & $\begin{array}{l}-4.1 \\
-0.7\end{array}$ & $\begin{array}{l}6.5 \\
3.0\end{array}$ & $\begin{array}{r}11.5 \\
5.0\end{array}$ & $\begin{array}{l}5.7 \\
4.0\end{array}$ \\
\hline \multicolumn{6}{|c|}{ Cruise-ship passenger arrivals } \\
\hline $\begin{array}{l}\text { Antigua } \\
\text { Caribbean area }\end{array}$ & $\begin{array}{l}9.0 \\
9.1\end{array}$ & $\begin{array}{l}12.9 \\
12.7\end{array}$ & $\begin{array}{r}-2.9 \\
7.2\end{array}$ & $\begin{array}{r}-4.7 \\
4.0\end{array}$ & $\begin{array}{r}1.9 \\
-1.0\end{array}$ \\
\hline
\end{tabular}

Sources: Caribbean Tourism Organization; and Antiguan Department of Tourism.

1/ Total visitors excluding cruise-ship passenger arrivals. 
Table 35. Antigua and Barbuda: Exchange Rates

\begin{tabular}{|c|c|c|c|c|c|}
\hline & \multicolumn{3}{|c|}{ Units per ECS } & \multicolumn{2}{|c|}{$\begin{array}{r}\text { Effective Exchange } \\
1980=100 \\
\end{array}$} \\
\hline & $\overline{\text { SDR }}$ & US\$ & $\tilde{E}$ & Nominal & $\overline{\text { Real }} 2 /$ \\
\hline \multicolumn{6}{|c|}{ Quarterly average } \\
\hline \multicolumn{6}{|l|}{1990} \\
\hline I & 0.282 & 0.370 & 0.223 & 110.5 & 98.8 \\
\hline II & 0.282 & 0.370 & 0.221 & 110.6 & 97.0 \\
\hline III & 0.270 & 0.370 & 0.199 & 107.4 & 95.2 \\
\hline IV & 0.258 & 0.370 & 0.190 & 105.7 & 95.4 \\
\hline \multicolumn{6}{|l|}{1991} \\
\hline$I$ & 0.262 & 0.370 & 0.194 & 106.4 & 95.9 \\
\hline II & 0.277 & 0.370 & 0.217 & 110.0 & 99.3 \\
\hline III & 0.266 & 0.370 & 0.220 & 110.6 & 99.6 \\
\hline IV & 0.267 & 0.370 & 0.209 & 109.8 & 98.3 \\
\hline \multicolumn{6}{|l|}{1992} \\
\hline$I$ & 0.267 & 0.370 & 0.209 & 110.5 & 98.3 \\
\hline II & 0.266 & 0.370 & 0.205 & 110.5 & 98.0 \\
\hline III & 0.255 & 0.370 & 0.194 & 108.3 & 95.9 \\
\hline IV & 0.264 & 0.370 & 0.234 & 113.1 & 100.1 \\
\hline \multicolumn{6}{|l|}{1993} \\
\hline $\bar{I}$ & 0.269 & 0.370 & 0.251 & 114.7 & 101.6 \\
\hline II & 0.262 & 0.370 & 0.241 & 114.8 & 101.9 \\
\hline III & 0.264 & 0.370 & 0.246 & 116.0 & 102.6 \\
\hline IV & 0.266 & 0.370 & 0.248 & 117.0 & 103.4 \\
\hline \multicolumn{6}{|l|}{1994} \\
\hline $\bar{I}$ & 0.267 & 0.370 & 0.249 & 117.7 & 104.1 \\
\hline II & 0.262 & 0.370 & 0.246 & 117.4 & 103.9 \\
\hline III & 0.254 & 0.370 & 0.239 & 115.9 & 102.5 \\
\hline IV & 0.253 & 0.370 & 0.233 & 115.2 & 101.9 \\
\hline \multicolumn{6}{|c|}{ Annual average } \\
\hline 1990 & 0.273 & 0.370 & 0.208 & 108.6 & 96.6 \\
\hline 1991 & 0.268 & 0.370 & 0.210 & 109.2 & 98.3 \\
\hline 1992 & 0.263 & 0.370 & 0.211 & 110.6 & 98.1 \\
\hline 1993 & 0.265 & 0.370 & 0.246 & 115.6 & 102.4 \\
\hline 1994 & 0.259 & 0.370 & 0.242 & 116.5 & 103.1 \\
\hline
\end{tabular}

Sources: IMF, International Financial Statistics; and Fund staff estimates.

1) The weighting scheme used to calculate indices of nominal and real effective exchange rate is based on data for tourism receipts and on data for aggregate bilateral non-oil trade flow.

2) Deflated by relative consumer prices. 
Table 36. Antigua and Barbuda: Terms of External Debt

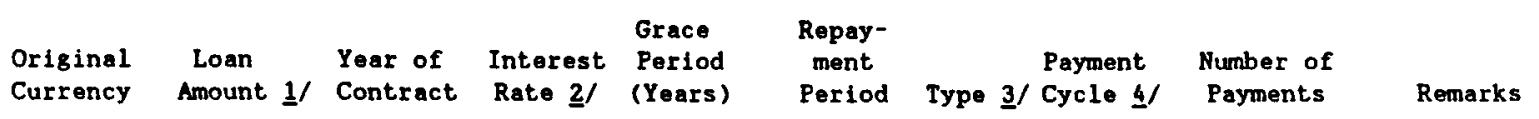

\section{Central Government}

Caribbean Development Bank

Emergency Fund assistance (1/EF)

Period Type 3/ Cycle $4 /$ Payments

Remarks

$\begin{array}{rrrr}\text { ECS } & 1,000 & 1976 & 6.00 \\ \text { C\$;EStB; US\$ } & 3,187 & 1979 & 5.00 \\ \text { USS } & 100 & 1973 & 4.00 \\ \text { CS } & 184 & 1974 & 4.00 \\ \text { USS } & 81 & 1979 & 4.00\end{array}$

\begin{tabular}{|c|c|c|c|c|c|c|c|c|}
\hline 6,378 & 1983 & 4.00 & $\ldots$ & $\ldots$ & $A$ & $Q$ & $\ldots$ & $\begin{array}{l}\text { Serviced out of U.S. } \\
\text { base lease receipts. }\end{array}$ \\
\hline 750 & 1983 & 6.00 & $\cdots$ & $\cdots$ & A & $\mathbf{Q}$ & $\ldots$ & \\
\hline 495 & 1979 & 1.00 & 10 & $1990-2019$ & $\mathbf{L}$ & B & 60 & \\
\hline 1,542 & 1985 & 1.00 & 10 & $1996-2025$ & L & B & 60 & \\
\hline 1,500 & 1990 & 2.00 & 5 & $1997-2018$ & $\mathrm{~L}$ & $\mathbf{B}$ & 40 & \\
\hline 250 & 1975 & 3.00 & 10 & $1985-2010$ & A & A & 25 & \\
\hline 3,825 & 1981 & 4.00 & 5 & $1984-94$ & A & B & 20 & \\
\hline 455 & 1981 & 4.00 & 5 & $1985-2004$ & A & B & 40 & \\
\hline 706 & 1967 & 6.28 & 1 & $1968-92$ & A & $A$ & 24 & \\
\hline 282 & 1981 & -- & -- & $1996-2010$ & $\mathrm{~L}$ & B & 28 & \\
\hline 1,000 & 1982 & 6.00 & $\underline{2}$ & $\underline{1985-90}$ & $\underline{L}$ & $\underline{B}$ & $\underline{10}$ & \\
\hline$\underline{7,000}$ & $\underline{1993}$ & 4.50 & $\underline{10}$ & $\underline{2003}$ & $\underline{L}$ & $\underline{\mathrm{B}}$ & $\underline{1}$ & \\
\hline 5,000 & 1987 & -- & 20 & $2007-2017$ & $L$ & $A$ & 10 & \\
\hline 20.000 & 1991 & -- & 20 & $2011-2031$ & $\mathrm{~L}$ & A & 10 & \\
\hline 15,786 & 1990 & -- & -- & $1990-95$ & $\mathrm{~L}$ & B & $\ldots$ & ) Serviced out of gaso- \\
\hline 22,000 & 1990 & -- & -- & $1990-95$ & $\mathbf{L}$ & B & $\ldots$ & Mine tax proceeds \\
\hline 425 & 1979 & 7.30 & 1 & $1981-85$ & $\mathbf{L}$ & B & 10 & \\
\hline 427 & 1980 & 7.30 & 1 & $1981-87$ & L & B & 10 & \\
\hline 172 & 1980 & 6.58 & 2 & $1982-85$ & $\mathbf{L}$ & B & 10 & \\
\hline 5,100 & 1981 & 10.00 & 1 & $1982-86$ & L & B & 8 & \\
\hline 3,000 & 1981 & 7.07 & 1 & $1983-86$ & $\mathbf{L}$ & B & 10 & \\
\hline
\end{tabular}

\section{China}

Sugar industry (2/SFR-OR)

Livestock development (2/SFR)

Cargo facility ( $3 / \mathrm{SFR}$ )

Feeder roads (17/SFR)

Fisheries development (18/SFR)

\section{U.S. Eximbank}

Colt Industries and Aqua Chem. (APUA)

Antigua Port Authority - Loan B

European Development Fund

North Shore Water Distribution

Road Rehabilitation Program I

Airport Navigation System

\section{Irinidad and Tobabo}

Earthquake damage

Rehabilitation of electricity supply

Factory shells

DM; USS : SKI;CS $\quad 3.271 \quad 1980 \quad 4.00$

United Kingdom

Independence

OPEC: Balance of payments support

Kuwaiti Fund - Road improvement

Creekside bridge

Exhibition center

\section{Commercial}

Credit Suisse - rescheduled

Foster Wheeler Power Prod. (Resch. 2)

Pye TV Ltd.

Italian International Bank

Lloyds Bank (Bahamas) - Carmichael

Banco do Brasil - Deep Bay

Loan "A"

$1979-94$

$1986-2001$

Loan " $B$ " 
Tablo 36. Antigua and Barbuda: Terms of Externel Debt (Continued)

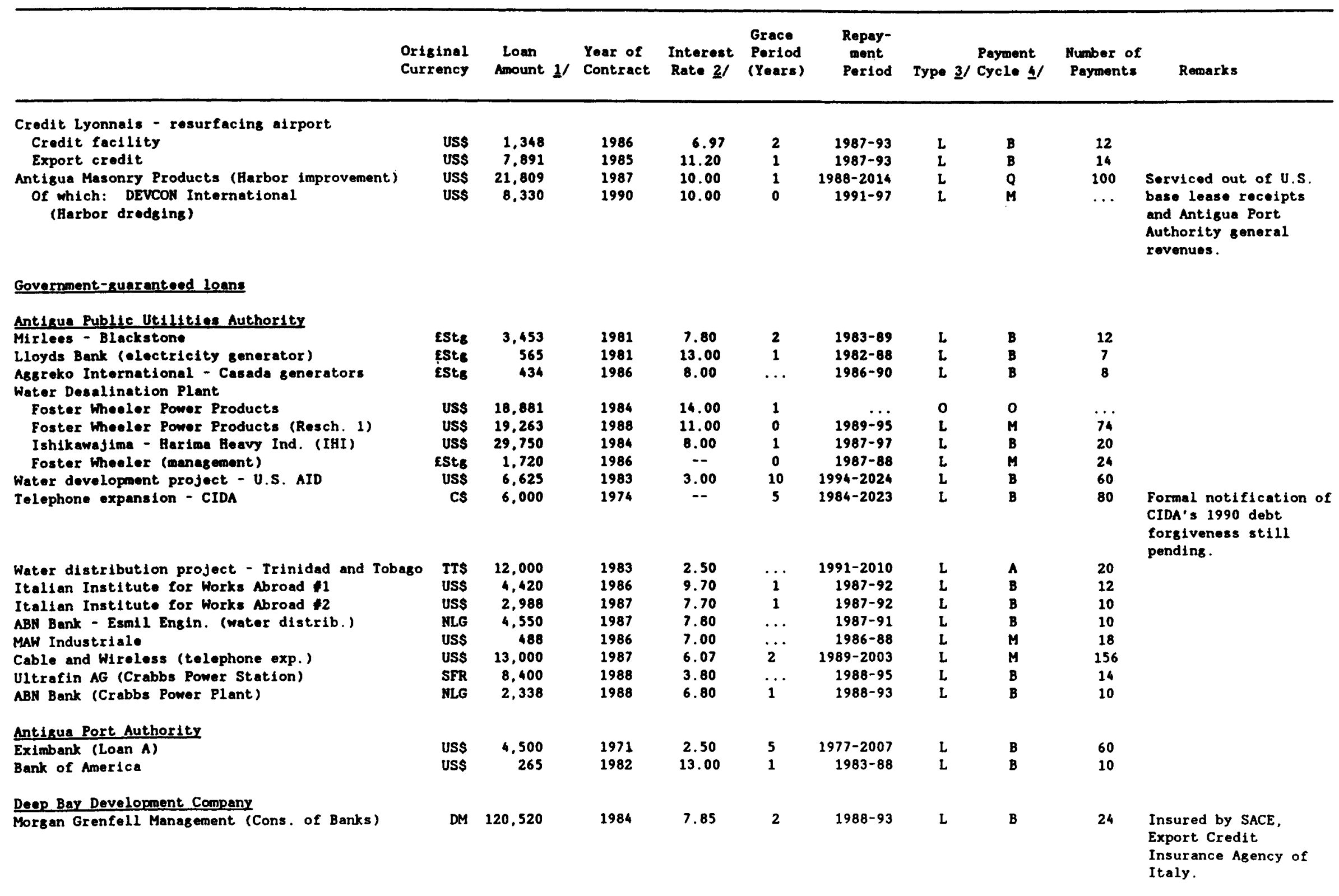


Table 36. Antigua and Barbuda: Terms of External Debt (Concluded)

\begin{tabular}{|c|c|c|c|c|c|c|c|c|c|c|}
\hline & $\begin{array}{l}\text { Original } \\
\text { Currency }\end{array}$ & $\begin{array}{l}\text { Loan } \\
\text { Amount } 1 /\end{array}$ & $\begin{array}{l}\text { Year of } \\
\text { Contract }\end{array}$ & $\begin{array}{l}\text { Interest } \\
\text { Rate } 2 /\end{array}$ & $\begin{array}{l}\text { Grace } \\
\text { Period } \\
\text { (Years) }\end{array}$ & $\begin{array}{l}\text { Repay- } \\
\text { ment } \\
\text { Period }\end{array}$ & Type $\underline{3} /$ & $\begin{array}{l}\text { Payment } \\
\text { Cycle } 4 /\end{array}$ & $\begin{array}{l}\text { Number of } \\
\text { Payments }\end{array}$ & Remarks \\
\hline \multicolumn{11}{|l|}{ St. John's Development Corporation } \\
\hline Ultrafin AG (Main) & DM & 25,075 & 1986 & 6.65 & 1 & $1989-94$ & $\mathrm{~L}$ & B & 10 & \\
\hline Ultrafin AG (Bridging) & DM & 393 & 1986 & 6.65 & -- & 1989 & L & A & 1 & \\
\hline \multicolumn{11}{|l|}{ Antigua and Barbuda Development Bank } \\
\hline Venezuela Investment Fund & US\$ & 500 & 1982 & 8.00 & $\ldots$ & $\ldots$ & $\mathbf{0}$ & B & $\ldots$ & \\
\hline \multicolumn{11}{|l|}{ Caribbean Developpent Bank } \\
\hline $\begin{array}{l}\text { Agricultural and industrial credit } I(2 / 0 R) \\
\text { Agricultural and industrial credit }\end{array}$ & $\mathrm{c} \$$ & 111 & 1974 & 7.30 & 5 & $1981-96$ & $\mathbf{L}$ & $\mathbf{Q}$ & 60 & \\
\hline $\operatorname{II}(3 / 0 R)$ & E; EStB;TTS & 92 & 1975 & 7.50 & 5 & $1981-96$ & $A$ & $\mathbf{Q}$ & 60 & \\
\hline Housing rehabilitation (1/SFR-OR) & ECS;USS;TIS & 213 & 1975 & 4.50 & 2 & $1978-88$ & A & Q & 40 & \\
\hline Mortgage finance ( $3 / \mathrm{SFR}-\mathrm{OR})$ & ;TS;EC\$;C\$ & 1,000 & 1980 & 6.80 & 5 & $1984-2001$ & $\mathbf{L}$ & $\mathbf{Q}$ & 60 & \\
\hline Farm improvement credit $(1 / \mathrm{SFR})$ 1st & cs & 200 & 1974 & 4.00 & 5 & $1980-95$ & L & B & 30 & \\
\hline Farm improvement credit (1/SFR) CCF & TTS & 94 & 1976 & 4.00 & 3 & $1980-95$ & $\mathrm{~L}$ & B & 30 & \\
\hline Student loans (4/SFR) & US\$ & 80 & 1974 & 4.00 & 5 & $1981-91$ & L & B & 20 & \\
\hline Industrial estates (5/SFR) & cs. & 197 & 1974 & 4,00 & 5 & $1981-96$ & A & Q & 60 & \\
\hline Small industry credit ( $6 / S F R)$ & TTS.USS & 73 & 1974 & 4.00 & 5 & $1980-95$ & $\mathbf{L}$ & 0 & 60 & \\
\hline Small industry credit II (B/SFR) & DM & 408 & 1976 & 4.00 & 4 & $1981-96$ & L & $Q$ & 60 & \\
\hline Farm improvement credit II $(9 /$ SFR) & c\$ & 191 & 1976 & 4.00 & 5 & $1981-96$ & A & Q & 60 & \\
\hline Industrial estates ( $12 / \mathrm{SFR})$ & ES; fStg; USS & 1,122 & 1977 & 4.00 & 5 & $1982-97$ & A & Q & 60 & \\
\hline Student loans (13/SFR) & ES;EStg;USS & 180 & 1977 & 4.00 & 5 & $1983-93$ & A & Q & 40 & \\
\hline Agricultural production credit (15/SFR) & US\$ & 192 & 1978 & 4.00 & 5 & $1983-98$ & A & Q & 60 & \\
\hline Agricultural and industrial credit (16/SFR) & EStg & 225 & 1978 & 4.00 & 4 & $1984-99$ & A & Q & 60 & \\
\hline Global line of credit (19/SFR) & us\$ & 800 & 1980 & 4.00 & 5 & $1986-2001$ & $\mathrm{~L}$ & $Q$ & 60 & \\
\hline Investment in equity (20/SFR) & us\$ & 800 & 1980 & 0.80 & 5 & $1990-2010$ & L & B & 40 & \\
\hline \multicolumn{11}{|l|}{ Central Housing and Planning Authority } \\
\hline CDB - UWCH (10/SFR) & uss & 560 & 1976 & 4.00 & 1 & $1978-98$ & A & 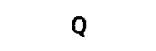 & 80 & \\
\hline \multicolumn{11}{|l|}{ Friends of the English Harbour } \\
\hline CDB loan $1 / O R$ & ECS & 510 & 1975 & 8.00 & 7 & $1982-93$ & A & B & 24 & \\
\hline CDB loan $1 / O R$ (additional) & US\$ & 73 & 1975 & 9.50 & 7 & $1982-93$ & $A$ & B & 24 & \\
\hline
\end{tabular}

Source: Ministry of Finance.

1/ In thousands of original currency.

2f Average rate in 1994 .

$3 /$ A - Annuity; L - Level payment; S - Sinking fund; and 0 - Other.

4/ A - Annual; B - Biannual; $Q$ - Quarterly; and M - Monthly. 
Table 37. Antigua and Barbuda: Extornal Debt Excluding Interest Arrears

(In thousends of U.S, dollern)

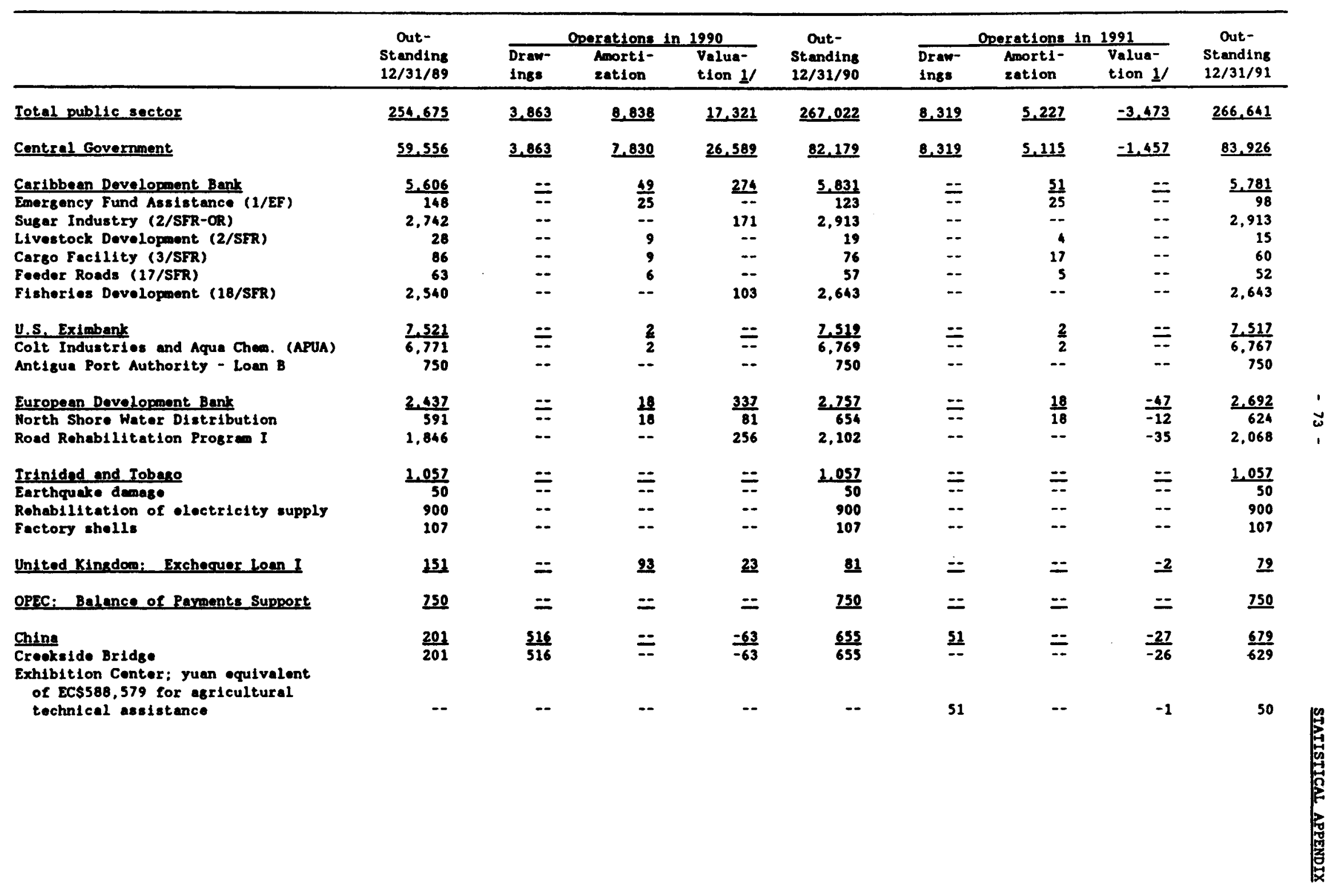


(In thousands of U.S, dollers)

\begin{tabular}{|c|c|c|c|c|c|c|c|c|c|}
\hline & \multirow{2}{*}{$\begin{array}{c}\text { Out- } \\
\text { Standing } \\
12 / 31 / 89\end{array}$} & \multicolumn{3}{|c|}{ Operations in 1990} & \multirow{2}{*}{$\begin{array}{c}\text { Out- } \\
\text { Standing } \\
12 / 31 / 90\end{array}$} & \multicolumn{3}{|c|}{ Operations in 1991} & \multirow{2}{*}{$\begin{array}{c}\text { Out- } \\
\text { Standing } \\
12 / 31 / 91\end{array}$} \\
\hline & & $\begin{array}{l}\text { Draw- } \\
\text { Ings }\end{array}$ & $\begin{array}{l}\text { Amort1- } \\
\text { zation }\end{array}$ & $\begin{array}{l}\text { Value- } \\
\text { tion 1/ }\end{array}$ & & $\begin{array}{l}\text { Draw- } \\
\text { Ings }\end{array}$ & $\begin{array}{l}\text { Amorti- } \\
\text { zation }\end{array}$ & $\begin{array}{l}\text { Valua- } \\
\text { tion } 1 / \\
\end{array}$ & \\
\hline Comercial & 41,833 & $\underline{3.347}$ & 7.668 & $\underline{26.018}$ & $\underline{63,529}$ & 8.268 & 5.044 & -1.382 & 65.371 \\
\hline Credit Suisse - Halcyon Cove & 8,186 & -- & -- & $\overline{-8,186}$ & & -- & -- & - & 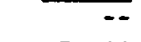 \\
\hline Credit Suisse - Rescheduled & -- & -- & 2,725 & 11,988 & 9,263 & -- & 837 & $-1,344$ & 7,082 \\
\hline Foster Wheeler Power Prod. (Resch. 2) & -- & -- & 4.000 & 22,000 & 18,000 & -- & 4,000 & -- & 14.000 \\
\hline Pye TV Ltd. & 477 & -- & -- & 96 & 573 & -- & - & -17 & 556 \\
\hline Italian International Bank & 596 & -- & -- & 120 & 715 & -- & -- & -21 & 694 \\
\hline Lloyds Bank (Baharoas) - Carmichael & 129 & -- & -- & -- & 129 & -- & - & -- & 129 \\
\hline Banco do Brasil - Deep Bay & 8,100 & -- & -- & -- & 8,100 & -- & -- & -- & 8,100 \\
\hline Loan "A" & 5,100 & -- & -- & -- & 5,100 & -- & -- & $\cdots$ & 5,100 \\
\hline Loan "B" & 3,000 & $\cdots$ & -- & -- & 3,000 & -- & - & -- & 3,000 \\
\hline Credit Lyonnais - resurfacing alrport & 0.145 & -- & -- & -- & 8,145 & -- & -- & -- & B, 145 \\
\hline Credit facility & 1.170 & -- & -- & -- & 1,170 & -- & -- & -- & 1,170 \\
\hline Export credit & 6,975 & -- & -- & -- & 6,975 & -- & -- & -- & 6.975 \\
\hline Antigua Masonry Products (harbor & & & & & & & & & \\
\hline $\begin{array}{l}\text { improvement) } \\
\text { of which: DEVCON International }\end{array}$ & 16,200 & 3,347 & 942 & -- & 18,605 & 8,268 & 208 & - & 26.665 \\
\hline (Harbor Dredging) & -- & 1,000 & -- & -- & 1,000 & 8,268 & -- & -- & 9,268 \\
\hline Goverment-suaranteed Loans & 195.119 & $=$ & 1.009 & $\underline{-9,267}$ & $\underline{184.843}$ & $\underline{z}$ & $\underline{112}$ & $\underline{-2.016}$ & 182.715 \\
\hline Antigua Public Utilities Authority & $\underline{100.233}$ & $=$ & $\underline{755}$ & $-21,080$ & 28,397 & $=$ & $=$ & $\underline{-592}$ & $\underline{77,806}$ \\
\hline Mirlees-Blackstone & 5,545 & -- & -- & 1,114 & 6,659 & -- & -- & -198 & 6.461 \\
\hline Lloyds Bank (electricity generator) & 907 & -- & -- & 182 & 1,089 & -- & -- & -32 & 1.057 \\
\hline As8reko International - Casada senerators & 729 & -- & -- & 146 & 875 & -- & -- & -26 & 849 \\
\hline Water Desalination Plant & 49.013 & $=$ & $=$ & $-19,263$ & $\underline{29.750}$ & $=$ & $=$ & $=$ & $\underline{29,750}$ \\
\hline Foster Wheeler Power Prod. (Resch. 1) & 19.263 & - & -- & $-19,263$ & & -- & -- & -- & -- \\
\hline Ishikawajima-Harima Beavy Ind. (IBI) & 29.750 & -- & -- & -- & 29,750 & -- & -- & -- & 29.750 \\
\hline Water Development Project - U.S. AID & 6.625 & -- & -- & -- & 6,625 & -- & -- & -- & 6,625 \\
\hline Telephone Expansion - CIDA & 4.792 & -- & -- & -4.792 & -- & -- & -- & -- & -- \\
\hline Water distribution project - I\& $T$ & 2,856 & -- & -- & - & 2,856 & -- & -- & -- & 2,856 \\
\hline Itallan Institute for Works Abroad $\$ 1$ & 4,420 & -- & -- & -- & 4.420 & -- & -- & -- & 4,420 \\
\hline Italian Inst ttute for Works Abroad $\$ 2$ & 2,988 & -- & -- & -- & 2,988 & -- & -- & -- & 2,988 \\
\hline ABN Bank - Esmil Engin. (water distrib.) & 2,376 & -- & -- & 317 & 2,692 & -- & -- & -32 & 2.660 \\
\hline MAW Industriale & 330 & -- & -- & -- & 330 & -- & -- & -- & 330 \\
\hline Cable and Wireless (telephone expansion) & 13,000 & -- & 755 & -- & 12,245 & -- & -- & -- & 12,245 \\
\hline U1trafin AG (Crabbs Power Station) & 5,431 & -- & -- & 1,053 & 6.484 & -- & -- & -287 & 6.197 \\
\hline ABN Bank (Crabbs Powor Plant) & 1,221 & -- & -- & 163 & 1,383 & -- & -- & -16 & 1,367 \\
\hline
\end{tabular}


Table 37. Antigua and Barbuda: External Debt Excluding Interest Arrears (Continued)

(In thousands of U.S. dollars)

\begin{tabular}{|c|c|c|c|c|c|c|c|c|c|}
\hline & \multirow{2}{*}{$\begin{array}{c}\text { Out- } \\
\text { Standins } \\
12 / 31 / 89\end{array}$} & \multicolumn{3}{|c|}{ Operations in 1990} & \multirow{2}{*}{$\begin{array}{c}\text { Out- } \\
\text { Standing } \\
12 / 31 / 90\end{array}$} & \multicolumn{3}{|c|}{ Operations in 1991} & \multirow{2}{*}{$\begin{array}{c}\text { Out- } \\
\text { Standing } \\
12 / 31 / 91\end{array}$} \\
\hline $\begin{array}{l}\text { Stat } \\
12 /:\end{array}$ & & $\begin{array}{l}\text { Draw- } \\
\text { ings }\end{array}$ & $\begin{array}{l}\text { Amort1- } \\
\text { zation }\end{array}$ & $\begin{array}{l}\text { Valua- } \\
\text { tion } 1 /\end{array}$ & & $\begin{array}{l}\text { Draw- } \\
\text { ings }\end{array}$ & $\begin{array}{l}\text { Amort1- } \\
\text { sation }\end{array}$ & $\begin{array}{l}\text { Valua- } \\
\text { tion 1/ }\end{array}$ & \\
\hline$\frac{\text { Antigua Port Authority }}{\text { U.S. Eximbank (Loan } A)}$ & $\frac{4,500}{4,500}$ & $\underline{--}$ & $=$ & $\underline{-=}$ & $\frac{4.500}{4,500}$ & $\ddot{-=}$ & $\ddot{-=}$ & $=$ & $\frac{4,500}{4,500}$ \\
\hline$\frac{\text { Deep Bay Development Company }}{\text { Morgan Gronfe11. Ment. (Cons. of Banks) }}$ & $\frac{70,986}{70,986}$ & $\ddot{=}$ & $=$ & $\frac{9.683}{9.683}$ & $\frac{80.669}{80,669}$ & $=$ & $\ddot{=-}$ & $\frac{-1,171}{-1,171}$ & $\frac{79.499}{79.499}$ \\
\hline St John's Devolopment Corporation & $\frac{15.002}{14.769}$ & $\underline{=}$ & $=$ & $\frac{2.046}{2,015}$ & $\frac{17,046}{16,784}$ & $=$ & $=$ & $\frac{-247}{-244}$ & $\frac{16,801}{16,540}$ \\
\hline UItrafin AG (Bridging) & 233 & - & $\cdots$ & 32 & 264 & $\cdots$ & -- & -4 & 261 \\
\hline $\begin{array}{l}\text { Antigue and Barbude Development Bank } \\
\text { Venezuela Investmont Fund }\end{array}$ & $\frac{3.773}{500}$ & $\underline{=}$ & $\frac{223}{\ldots-}$ & $\frac{86}{--}$ & $\frac{3.634}{500}$ & $\underline{-=}$ & $\underline{81}$ & $\underline{-6}$ & $\frac{3,546}{500}$ \\
\hline Cartbbean Developmont Bank & 3,273 & -- & 223 & 84 & 3,134 & -- & 81 & -6 & 3,046 \\
\hline Agricultural and Industrial Credit $I(2 / 0 R)$ & 53 & -- & -- & -- & 53 & -- & -- & -- & 53 \\
\hline Agricultural and Industrial Credlt II(3/OR) & 44 & -- & -- & 2 & 46 & $\cdots$ & 2 & -- & 44 \\
\hline Bousing Rehabilitation (1/SFR-OR) & 49 & - & 10 & -- & 39 & -- & -- & -- & 39 \\
\hline Mortgage Finance (3/SFR-OR) & 526 & -- & -- & -53 & 673 & -- & 3 & -- & 470 \\
\hline Farm Improvement Credit (1/SFR) 1st & 63 & - & 6 & -- & 37 & -- & 12 & -- & 46 \\
\hline Farm Improvenont Credit (1/SFR) CCF & 14 & -- & -- & -- & 14 & - & 1 & -- & 13 \\
\hline Student Loans (4/SFR) & 16 & -- & 7 & -- & 9 & -- & -- & -- & $\mathbf{9}$ \\
\hline Induatrial Estatos (5/SFR) & 125 & -- & 9 & -- & 115 & -- & -- & -- & 116 \\
\hline Smell Industry Crodit ( $6 / \mathrm{SFR})$ & 35 & -- & -. & -- & 35 & -- & 12 & -- & 23 \\
\hline Sma11 Industry Credit II (8/SFR) & 148 & -- & -- & 20 & 169 & -- & -- & -2 & 166 \\
\hline Farm Improvement Credit II ( $9 / \mathrm{SFR})$ & 91 & -- & -- & -- & 90 & -- & -- & -- & 91 \\
\hline Industrial Estates (12/SFR) & 834 & -- & 45 & 79 & 868 & -- & -- & -- & 868 \\
\hline Student Loans (13/SFR) & 194 & -- & 77 & 12 & 79 & -- & -- & -- & 79 \\
\hline Agricultural Production Credit (15/SFR) & 107 & -- & 8 & -- & 99 & -- & 11 & -- & 88 \\
\hline Asriculturel and Industriel Crodit (16/SFR) & ) 124 & $\cdots$ & 9 & 24 & 139 & -- & 15 & -5 & 119 \\
\hline Global Line of Credit (19/SFR) & 342 & -- & 45 & -- & 297 & -- & 24 & -- & 273 \\
\hline Investmont in Equity (20/SFR) & 558 & -- & 6 & -- & 552 & -- & - & $\cdots$ & 552 \\
\hline Central Housins and Planning Authority & $\underline{301}$ & $=$ & $\underline{31}$ & $=$ & $\underline{270}$ & $=$ & $\underline{31}$ & $=$ & $\underline{239}$ \\
\hline CDB - UWCE (10/SFR) & $\overline{301}$ & $\overline{--}$ & $\overline{31}$ & $\overline{--}$ & 270 & $\overline{--}$ & $\overline{31}$ & $=$ & $\overline{239}$ \\
\hline Friends of the Enslish Barbour & $\underline{324}$ & $=$ & $=$ & $=$ & $\underline{324}$ & $=$ & $=$ & $=$ & $\underline{324}$ \\
\hline CDB loan 1/OR & $\overline{242}$ & $\overline{--}$ & $=$ & $=$ & 242 & $\overline{--}$ & $\overline{-}$ & $\overline{--}$ & 242 \\
\hline CDB loan 1/OR (additional) & 82 & -- & -- & -- & 82 & -- & -- & -- & 82 \\
\hline
\end{tabular}


Table 37. Antigue and Barbuda: External Debt Excluding Interest Arrears (Continued)

(In thous ands of U.S. dollars)

\begin{tabular}{|c|c|c|c|c|c|c|c|c|c|}
\hline & \multirow{2}{*}{$\begin{array}{c}\text { Out- } \\
\text { Standing } \\
12 / 31 / 91\end{array}$} & \multicolumn{3}{|c|}{ Operations in 1992} & \multirow{2}{*}{$\begin{array}{c}\text { Out- } \\
\text { Standing } \\
12 / 31 / 92\end{array}$} & \multicolumn{3}{|c|}{ Operations in 1993} & \multirow{2}{*}{$\begin{array}{c}\text { Out- } \\
\text { Standing } \\
12 / 31 / 93\end{array}$} \\
\hline & & $\begin{array}{l}\text { Draw- } \\
\text { ings }\end{array}$ & $\begin{array}{l}\text { Amorti- } \\
\text { zation }\end{array}$ & $\begin{array}{l}\text { Valua- } \\
\text { tion } 1 /\end{array}$ & & $\begin{array}{l}\text { Draw- } \\
\text { Ings }\end{array}$ & $\begin{array}{l}\text { Amorti- } \\
\text { 2ation }\end{array}$ & $\begin{array}{l}\text { Valua- } \\
\text { tion } \underline{1}\end{array}$ & \\
\hline Iotal public sector & $\underline{266.641}$ & $\underline{214}$ & $\underline{5.814}$ & $-10,027$ & $\underline{251.014}$ & $=$ & $\underline{5.448}$ & $\underline{-8,529}$ & $\underline{237,037}$ \\
\hline Central Government & 83.926 & $\underline{214}$ & 4,921 & $-1,864$ & $\underline{77.355}$ & $=$ & $\underline{5.141}$ & $-1,382$ & $\underline{70.832}$ \\
\hline $\begin{array}{l}\text { Caribbean Development Bank } \\
\text { Emergency Fund Assistance (1/EF) }\end{array}$ & $\frac{5.781}{98}$ & $=$ & $\frac{49}{25}$ & $\frac{-5}{--}$ & $\frac{5.727}{74}$ & $\overline{--}$ & $\frac{38}{25}$ & $\frac{-2}{--}$ & $\frac{5.688}{49}$ \\
\hline Sugar Industry (2/SFR-OR) & 2,913 & -- & -- & -- & 2,913 & -- & - & - & 2,913 \\
\hline Livestock Development ( $2 / \mathrm{SFR})$ & 15 & -- & 5 & -- & 10 & -- & 7 & -- & 3 \\
\hline Cargo Facility (3/SFR) & 60 & -- & 16 & -5 & 39 & -- & -- & -2 & 37 \\
\hline Foeder Roads (17/SFR) & 52 & -- & 3 & -- & 49 & -- & 7 & - & 42 \\
\hline Fisheries Development (18/SFR) & 2,643 & -- & -- & -- & 2,643 & -- & -- & -- & 2,643 \\
\hline U.S. Eximbank & 7.517 & $=$ & $=$ & $=$ & 2.517 & $\underline{z}$ & $=$ & $=$ & 7,517 \\
\hline Colt Industries and Aqua Chem. (APUA) & $\overline{6,767}$ & $\overline{--}$ & $=$ & $\overline{--}$ & $\overline{6,767}$ & $\overline{--}$ & $\overline{--}$ & $\overline{-\infty}$ & $\overline{6,767}$ \\
\hline Port Authority - Loan B & 750 & -- & -- & -- & 750 & -- & -- & -- & 750 \\
\hline European Deve lopment Bank & 2.692 & $=$ & $\underline{19}$ & -260 & 2.414 & $=$ & $\underline{9}$ & -181 & $\underline{2.224}$ \\
\hline North Shore Water Distribution & 624 & $=$ & 19 & -59 & 546 & $\overline{-}$ & 9 & -41 & 497 \\
\hline Road Rehabilitation Prosram I & 2,068 & - & -- & -200 & 1,867 & -- & -- & -140 & 1,727 \\
\hline Irinidad and Tobero & 1.057 & $=$ & $=$ & $=$ & $\underline{1.057}$ & $=$ & $=$ & -284 & $\underline{773}$ \\
\hline Earthquake damage & 50 & $\overline{--}$ & $=$ & $=$ & 50 & $\overline{--}$ & $=-$ & $\overline{-13}$ & 36 \\
\hline Rehabilitation of electricity supply & 900 & -- & -- & -- & 900 & -- & -- & -242 & 658 \\
\hline Factory shells & 107 & -- & -- & -- & 107 & -- & -- & -29 & 78 \\
\hline United Kingdom & $\underline{79}$ & $\underline{145}$ & $=$ & -36 & $\underline{188}$ & $=$ & $=$ & -4 & $\underline{183}$ \\
\hline United Kingdom: Exchequer Loan I & $\overline{79}$ & $=$ & $=$ & $\overline{-15}$ & $\overline{64}$ & $=$ & $=-$ & $\overline{-1}$ & 62 \\
\hline United Kingdom: Independence loan & -- & 145 & - & -21 & 124 & -- & -- & -3 & 121 \\
\hline OPEC: Balence of Payments Support & $\underline{750}$ & $=$ & $=$ & $=$ & $\underline{750}$ & $=$ & $=$ & $=$ & $\underline{750}$ \\
\hline China & & & & & & & & & \\
\hline Creekside Bridge & 629 & 69 & -- & -38 & 661 & -- & -- & -5 & 655 \\
\hline technicel assistance & 50 & -- & -- & -3 & 48 & -- & -- & -- & 47 \\
\hline
\end{tabular}


Table 37. Antigue and Barbuda: Externel Debt Excluding Interest Arrears (Continued)

(In thousands of U.S, dollars)

\begin{tabular}{|c|c|c|c|c|c|c|c|c|c|}
\hline & \multirow{2}{*}{$\begin{array}{c}\text { Out- } \\
\text { Standing } \\
12 / 31 / 91\end{array}$} & \multicolumn{3}{|c|}{ Operations in 1992} & \multirow{2}{*}{$\begin{array}{c}\text { Out- } \\
\text { Standing } \\
12 / 31 / 92\end{array}$} & \multicolumn{3}{|c|}{ Operations in 1993} & \multirow{2}{*}{$\begin{array}{c}\text { Out- } \\
\text { StandIng } \\
12 / 31 / 93\end{array}$} \\
\hline & & $\begin{array}{l}\text { Dran- } \\
\text { Ings }\end{array}$ & $\begin{array}{l}\text { Amorti- } \\
\text { zation }\end{array}$ & $\begin{array}{l}\text { Valua- } \\
\text { tion } 1 /\end{array}$ & & $\begin{array}{l}\text { Draw- } \\
\text { ings }\end{array}$ & $\begin{array}{l}\text { Amorti- } \\
\text { zation }\end{array}$ & $\begin{array}{l}\text { Valua } \\
\text { tion } 1 /\end{array}$ & \\
\hline$\frac{\text { Commercial }}{\text { Credit Suisse - Rescheduled }}$ & $\frac{65.371}{7,082}$ & $\equiv$ & $\frac{4.853}{853}$ & $\frac{-1,523}{-1,284}$ & $\frac{58,994}{4.945}$ & $\frac{-}{--}$ & $\frac{5.095}{812}$ & $\frac{-905}{-889}$ & $\frac{52.994}{3.244}$ \\
\hline Foster Wheeler Power Prod. (Resch. 2) & 14.000 & -- & 4.000 & 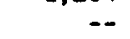 & 10,000 & -- & 4,000 & -- & 6,000 \\
\hline Pye TV Ltd. & 556 & -- & -- & -107 & 449 & -- & -- & -9 & 440 \\
\hline Italian International Bank & 694 & -- & -- & -133 & 561 & -- & 283 & -8 & 271 \\
\hline Lloyds Bank (Bahamas) - Carmichael & 129 & -- & -. & -- & 129 & -- & -- & -- & 129 \\
\hline Banco do Brasil - Deep Bay & 8,100 & -- & -- & -- & 8,100 & -- & - & -- & 8.100 \\
\hline Loan "A" & 5,100 & -- & -. & -- & 5,100 & -. & -- & -. & 5,100 \\
\hline Loan "B" & 3,000 & -- & -- & -- & 3,000 & -- & -- & -- & 3,000 \\
\hline Credit Lyonnais - resurfacing alrport & 8,145 & $\cdots$ & -- & -- & 8,145 & -- & $\cdots$ & -- & 8,145 \\
\hline Crodit facility & 1,170 & -- & .. & -. & 1,170 & -- & -- & -- & 1,170 \\
\hline Export credit & 6,975 & - & -- & -- & 6,975 & -- & -- & -- & 6,975 \\
\hline & & & & & & & & & \\
\hline Of which: DEVCON International & 26,665 & - & $\cdots$ & -- & 26,665 & $\cdots$ & $=-$ & -- & 26,665 \\
\hline (harbor dredging) & 9.268 & -- & -- & -- & 9,268 & -- & -- & -- & 9,268 \\
\hline Government-guaranteed loans & 182.715 & $=$ & $\underline{893}$ & -8.163 & $\underline{173.659}$ & $=$ & $\underline{\mathbf{3 0 7}}$ & $-7,147$ & $\underline{166,205}$ \\
\hline Antisue Public Utilities Authority & $\underline{77.806}$ & $=$ & $=$ & $-2,262$ & 75.543 & $=$ & $=$ & $-1,246$ & 74,297 \\
\hline Mirlees-Blackstone & $\overline{6,461}$ & $\overline{-}$ & $\overline{--}$ & $-1,239$ & 5,222 & $\overline{-}$ & $=$ & -106 & 5,116 \\
\hline Lloyds Bank (electricity generator) & 1,057 & -- & - & -203 & 854 & -- & -- & -17 & 837 \\
\hline Agrreko Internetionel - Cesada generators & 849 & -- & -- & -163 & 686 & -- & -- & -14 & 672 \\
\hline Water Desalination Plant & 29,750 & - & -- & -- & 29.750 & - & -- & -- & 29.750 \\
\hline Ishikawajima-Harima Heavy Ind. (IHI) & 29,750 & -- & -- & -- & 29,750 & -- & -- & - & 29.750 \\
\hline Water Dovolopment Project - U.S. AID & 6.625 & -- & -- & -- & 6,625 & -- & -- & -- & 6,625 \\
\hline Water distribution project $-I \& T$ & 2,856 & -- & -- & - & 2,856 & -- & -- & -768 & 2,088 \\
\hline Italian Institute for Works Abroad 1 & 4,420 & $\cdots$ & -- & -- & 4,420 & -- & -- & -- & 4,420 \\
\hline Italfan Institute for Works Abroad $\$ 2$ & 2,988 & -- & -- & -- & 2.988 & -- & -- & -- & 2,988 \\
\hline ABI Bank - Esmil Engln. (water distrib.) & 2,660 & -- & -- & -152 & 2,508 & -- & -- & -164 & 2,344 \\
\hline MaW Industriale & 330 & -- & -- & -- & 330 & -- & -- & -- & 330 \\
\hline Cable and Wireless (telephone oxpansion) & 12,245 & -- & -- & -- & 12,245 & -. & -- & -- & 12,245 \\
\hline U1trafin AG (Crabbs Powor Station) & 6,197 & -- & - & -428 & 5,769 & - & -- & -92 & 5,678 \\
\hline ABN Bank (Crabbs Power Plant) & 1,367 & - & -- & -78 & 1,289 & -- & -- & -84 & 1.205 \\
\hline
\end{tabular}


(In thousands of U.S, dollars)

\begin{tabular}{|c|c|c|c|c|c|c|c|c|c|}
\hline & \multirow{2}{*}{$\begin{array}{l}\text { Out- } \\
\text { Standins } \\
12 / 31 / 91\end{array}$} & \multicolumn{3}{|c|}{ Operattons in 1992} & \multirow{2}{*}{$\begin{array}{l}\text { Out- } \\
\text { Standing } \\
12 / 31 / 92\end{array}$} & \multicolumn{3}{|c|}{ Operations in 1993} & \multirow{2}{*}{$\begin{array}{l}\text { Out- } \\
\text { Standing } \\
12 / 31 / 93\end{array}$} \\
\hline $\begin{array}{l}\text { Stan } \\
12 / 3\end{array}$ & & $\begin{array}{l}\text { Draw- } \\
\text { ings }\end{array}$ & $\begin{array}{l}\text { Amorti- } \\
\text { zation }\end{array}$ & $\begin{array}{l}\text { Valua- } \\
\text { tion } 1 /\end{array}$ & & $\begin{array}{l}\text { Draw- } \\
\text { Inss }\end{array}$ & $\begin{array}{l}\text { Amorti- } \\
\text { zation }\end{array}$ & $\begin{array}{l}\text { Valua- } \\
\text { tion } 1 /\end{array}$ & \\
\hline$\frac{\text { Antigua Port Authority }}{\text { Eximbank (Loan A) }}$ & $\frac{4,500}{4,500}$ & $=$ & $\ddot{-=}$ & $\ddot{-\infty}$ & $\frac{4.500}{4.500}$ & $=$ & $\overline{-\sigma}$ & $\overline{-}$ & $\frac{4.500}{4,500}$ \\
\hline$\frac{\text { Deep Bey Development Company }}{\text { Morgan Grenfell. Mgmt. (Cons. of Banks) }}$ & $\frac{79,499}{79.499}$ & $\ddot{z}$ & 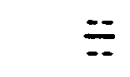 & $\frac{-4,827}{-4,827}$ & $\frac{74.672}{74.672}$ & $\ddot{=}$ & 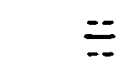 & $\frac{-4,858}{-4,858}$ & $\frac{69.814}{69.814}$ \\
\hline $\begin{array}{l}\text { St John's Development Corporation } \\
\text { U1trafin AG (Main) } \\
\text { ULtrafin AG (Bridging) }\end{array}$ & $\begin{array}{r}\frac{16.801}{16.540} \\
261\end{array}$ & $\begin{array}{ll}=- \\
--\end{array}$ & $\begin{array}{l}=- \\
--\end{array}$ & $\begin{array}{r}\frac{-1.020}{-1,004} \\
-16\end{array}$ & $\begin{array}{r}\frac{15.781}{15.536} \\
245\end{array}$ & $\begin{array}{l}=- \\
--\end{array}$ & $\begin{array}{l}= \\
=-\end{array}$ & $\begin{array}{r}\frac{-1.027}{-1.011} \\
-16\end{array}$ & $\frac{14.754}{14.525}$ \\
\hline $\begin{array}{l}\text { Antigua and Barbuda Development Bank } \\
\text { Venezuele Investment Fund } \\
\text { Carlbbeen Development Bank }\end{array}$ & $\begin{array}{r}\frac{3,546}{500} \\
3,046\end{array}$ & $\bar{z}$ & $\begin{array}{l}\frac{861}{101} \\
761\end{array}$ & $\frac{-53}{--}$ & $\begin{array}{r}\frac{2.632}{399} \\
2.233\end{array}$ & $=$ & $\begin{array}{r}\frac{282}{69} \\
213\end{array}$ & $\frac{-17}{-17}$ & $\begin{array}{r}\frac{2.333}{331} \\
2.002\end{array}$ \\
\hline $\begin{array}{l}\text { Agricultural and Industrial Credtt } I(2 / O R) \\
\text { Agricultural and Industrial Credit } I I(3 / 0 R)\end{array}$ & 53 & $\ddot{-}$ & 22 & -4 & $\begin{array}{l}27 \\
40\end{array}$ & -- & $\begin{array}{l}4 \\
3\end{array}$ & $\begin{array}{l}-1 \\
--\end{array}$ & $\begin{array}{l}23 \\
37\end{array}$ \\
\hline Housing Rehabilitation (1/SFR-OR) & 39 & - & 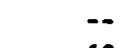 & -- & 39 & -- & -- & -- & 39 \\
\hline Mortgage Finance (3/SFR-OR) & 470 & -- & 68 & -- & 402 & -- & 25 & -- & 376 \\
\hline Farm Improvement Credit (1/SFR) 1st & 46 & -- & 17 & -3 & 26 & -- & 13 & -2 & 12 \\
\hline Farm Improvement Credit (1/SFR) CCF & 13 & -- & -- & -- & 12 & $\cdots$ & - & -3 & 9 \\
\hline Student Loans (4/SFR) & 9 & -- & 2 & -- & 7 & -- & -- & -- & 7 \\
\hline Industrial Estates (5/SFR) & 116 & -- & 39 & -9 & 68 & -- & 12 & -2 & 54 \\
\hline Small Industry Crod1t (6/SFR) & 23 & $\cdots$ & 6 & $\cdots$ & 17 & -- & 7 & -- & 10 \\
\hline Small Industry Crodit II (8/SFR) & 166 & -- & 70 & -8 & 88 & -- & 12 & -5 & 71 \\
\hline Farm Improvement Credit II (9/SFR) & 91 & -- & 16 & -7 & 68 & -- & -- & -3 & 65 \\
\hline Industrial Estetes (12/SFR) & 868 & -- & 390 & -. & 478 & -- & 84 & -- & 394 \\
\hline Student Loans (13/SFR) & 79 & -- & 56 & -- & 23 & -- & 16 & -- & 7 \\
\hline Agricultural Production Credit (15/SFR) & 88 & -- & 18 & -- & 70 & -- & 7 & -- & 63 \\
\hline Agricultural and Industrial Credit (16/SFR) & 119 & -- & 4 & -22 & 93 & -- & -- & -2 & 91 \\
\hline Globel Line of Credit (19/SFR) & 273 & -- & 34 & -- & 239 & -- & 30 & $\cdots$ & 209 \\
\hline Investment in Equity (20/SFR) & 552 & - & 16 & - & 536 & -- & -- & -- & 536 \\
\hline Central Housing and Plannins Authority & $\underline{239}$ & $=$ & $\underline{32}$ & $=$ & 207 & $=$ & $\underline{25}$ & $=$ & $\underline{182}$ \\
\hline CDB - UWCH (10/SFR) & $\overline{239}$ & - & $\overline{32}$ & $=$ & $\overline{207}$ & $=$ & $\overline{25}$ & $\overline{--}$ & $\overline{182}$ \\
\hline Friends of the English Rarbour & $\frac{324}{120}$ & $=$ & $=$ & $=$ & $\frac{324}{212}$ & $=$ & $=$ & $=$ & $\underline{324}$ \\
\hline CDB loan 1/OR & 242 & -- & -- & -- & 242 & -- & -- & -- & $\overline{242}$ \\
\hline CDB loan $1 / O R$ (additional) & 82 & - & -- & -- & 82 & -- & -- & -- & 82 \\
\hline
\end{tabular}


Table 37. Antigua and Barbuda: External Debt Excluding Interest Arrears (Continued)

(In thousands of U.S. dollars)

\begin{tabular}{|c|c|c|c|c|c|}
\hline & \multirow{2}{*}{$\begin{array}{l}\text { Out- } \\
\text { Standing } \\
12 / 31 / 93\end{array}$} & \multicolumn{3}{|c|}{ Operations in 1994} & \multirow{2}{*}{$\begin{array}{l}\text { Out- } \\
\text { Standing } \\
12 / 31 / 94\end{array}$} \\
\hline & & $\begin{array}{l}\text { Draw- } \\
\text { ings }\end{array}$ & $\begin{array}{l}\text { Amorti- } \\
\text { zation }\end{array}$ & $\begin{array}{l}\text { Valua- } \\
\text { tion } 1 /\end{array}$ & \\
\hline Total public sector & $\underline{237,037}$ & 1.646 & $\underline{5.284}$ & 10,719 & $\underline{244,119}$ \\
\hline Central Government & 20.832 & $\underline{1.646}$ & 4,916 & -461 & 67,102 \\
\hline Caribbean Development Bank & $\underline{5.688}$ & $=$ & $\underline{21}$ & $\underline{-2}$ & $\underline{5.665}$ \\
\hline Emergency Fund Assistance (1/EF) & 49 & - & $=-$ & $=$ & 49 \\
\hline Sugar Industry $(2 / S F R-O R)$ & 2,913 & -- & -- & -- & 2,913 \\
\hline Livestock Development (2/SFR) & 3 & -- & 3 & -- & $-\overline{-}$ \\
\hline Cargo Facility (3/SFR) & 37 & -- & 12 & -2 & 23 \\
\hline Foodor Roads (17/SFR) & 42 & -- & 5 & -- & 37 \\
\hline Fisheries Development (18/SFR) & 2,643 & -- & -- & -- & 2,643 \\
\hline U.S. Eximbank & 2.517 & $=$ & $=$ & $=$ & 7,517 \\
\hline Colt Industries and Aqua Chem. (APUA) & $\overline{6,767}$ & $\overline{--}$ & $\overline{--}$ & $\overline{--}$ & 6.767 \\
\hline Port Authority - Loan B & 750 & -- & -- & -- & 750 \\
\hline European Development Fund & $\underline{2.224}$ & $\underline{1.189}$ & 17 & $\underline{259}$ & $\underline{3.655}$ \\
\hline North Shore Water Distribution & 497 & -- & 17 & 48 & 528 \\
\hline Road Rehabilitation Program I & 1.727 & -- & -- & 170 & 1,897 \\
\hline Road Rohabilitation Program II & -- & 1.189 & -- & 41 & 1,230 \\
\hline Trinidad and Tobago & $\frac{773}{21}$ & $=$ & $=$ & $\underline{-10}$ & $\underline{763}$ \\
\hline Earthquake damage & 36 & -- & -- & -- & 36 \\
\hline Rohabilitation of electricity supply & 658 & - & -- & -9 & 649 \\
\hline Factory shells & 78 & -- & -- & -1 & 77 \\
\hline United Kingdom & $\underline{183}$ & $=$ & $=$ & 10 & 194 \\
\hline United Kingdam: Exchequer Loan I & 62 & -- & -- & 3 & 66 \\
\hline United Kingdom: Independence loan & 121 & -- & -- & 7 & 128 \\
\hline OPEC: Balance of Payments Support & $\underline{750}$ & $=$ & $=$ & $=$ & $\underline{750}$ \\
\hline Kuwait I Fund Road Improvement & $=$ & $\underline{458}$ & $=$ & $=$ & $\underline{458}$ \\
\hline Chine & $\underline{702}$ & $=$ & $=$ & -220 & $\underline{482}$ \\
\hline $\begin{array}{l}\text { Creokside Bridge } \\
\text { Exhibition Conter; yuan equivalont } \\
\text { of ECS588,579 for agricultural }\end{array}$ & $\overline{655}$ & $=$ & $=$ & -205 & $\overline{450}$ \\
\hline tochnical assistance & 47 & - & -- & -15 & 32 \\
\hline Commercial & $\underline{52.994}$ & $=$ & 4.877 & -498 & $\underline{47.619}$ \\
\hline Credit Suisse - Rescheduled & 3,244 & $\overline{--}$ & 877 & -537 & 1,830 \\
\hline Foster Wheeler Power Prod. (Resch. 2) & 6.000 & -- & 4,000 & -- & 2.000 \\
\hline Pye IV Ltd. & 440 & - & -- & 24 & 464 \\
\hline Italian International Bank & 271 & -- & -- & 15 & 286 \\
\hline Lloyds Bank (Bahamas) - Carmichael & 129 & -- & $=$ & -- & 129 \\
\hline Banco do Brasil - Deep Bay & 8,100 & - & -- & -- & 8,100 \\
\hline Loan "A" & 5,100 & -- & -- & - & 5,100 \\
\hline Loan "B" & 3.000 & -- & -- & -- & 3,000 \\
\hline Credit Lyonnais - Resurfacing airport & 8,145 & -- & -- & -- & 8,145 \\
\hline Credit facility & 1.170 & -- & -- & -- & 1,170 \\
\hline Export credit & 6,975 & $-\cdot$ & -- & -- & 6,975 \\
\hline Antigua Masonry Products & & & & & \\
\hline $\begin{array}{l}\text { (Harbor improvement) } \\
\text { Of which: DEVCON International }\end{array}$ & 26,665 & -- & -- & -- & 26,665 \\
\hline (Harbor dredging) & 9,268 & -- & -- & -- & 9.268 \\
\hline Government-guaranteed loans & 166,205 & $=$ & $\underline{368}$ & 11,180 & 177.017 \\
\hline
\end{tabular}


Table 37. Antigua and Barbuda: External Debt Excluding Interest Arrears (Concluded)

(In thousands of U.S. dollars)

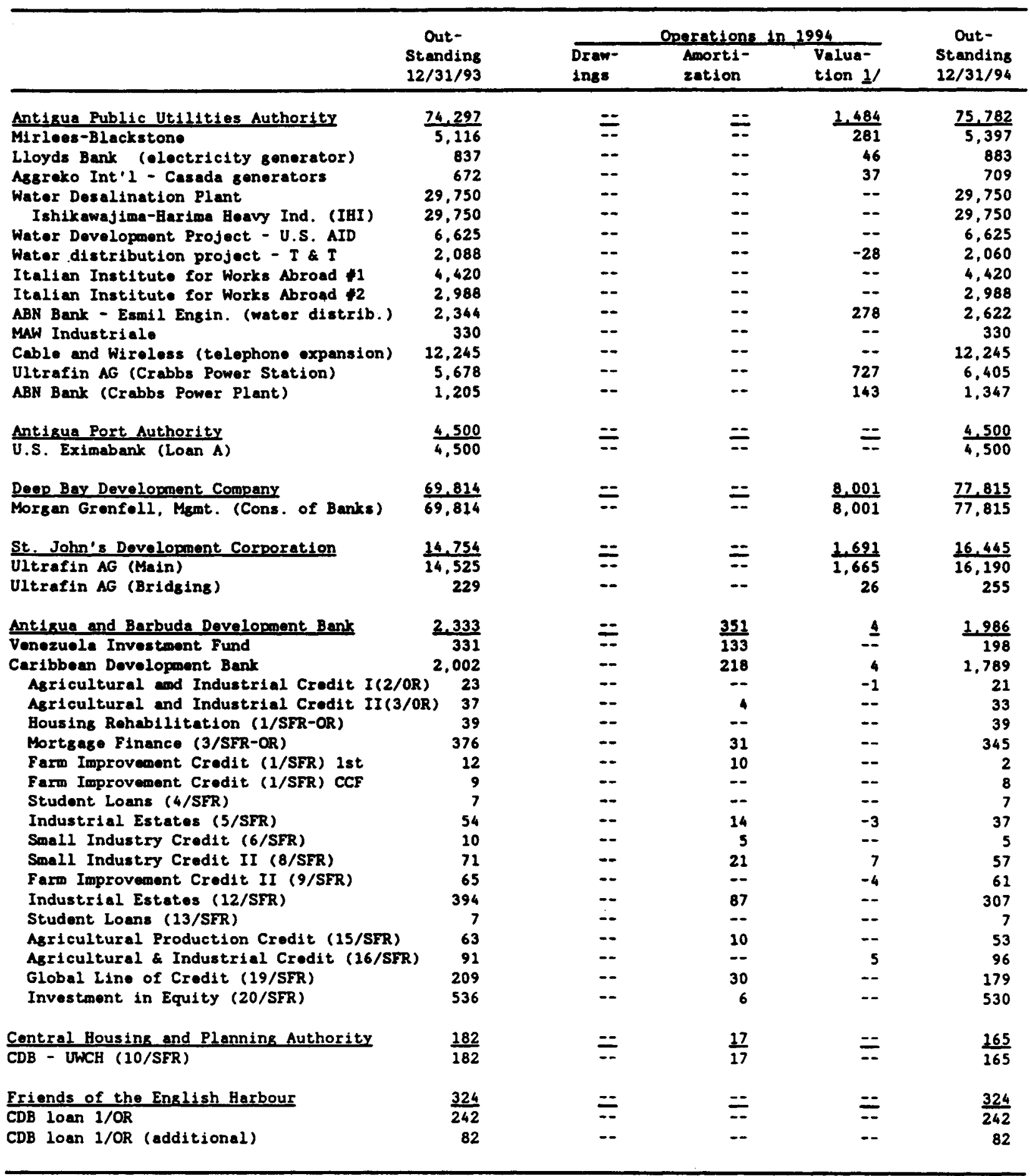

Source: Ministry of Finance.

1/ Includes debt reduction and rescheduling operations. 
(In thousends of U,S, dollars)

\begin{tabular}{|c|c|c|c|c|c|c|}
\hline & \multicolumn{3}{|c|}{1990} & \multicolumn{3}{|c|}{1991} \\
\hline & Principal & Interest & Total & Principal & Interest & Total \\
\hline \multicolumn{7}{|c|}{ (Outstand1ng at ond-year) } \\
\hline Total publlc sector & $\underline{101.966}$ & 61,536 & $\underline{163,503}$ & $\underline{127.654}$ & 85,048 & 212,702 \\
\hline Central Government & 17.639 & 17,009 & 34.648 & 20,896 & $\underline{19.918}$ & 40,815 \\
\hline Official & 4,603 & 3,566 & $\overline{8,169}$ & 5,391 & $\overline{3.964}$ & 9.355 \\
\hline Cartbbean Development Bank & 1,165 & 2.311 & 3,476 & 1,604 & 2,666 & 4.270 \\
\hline U.S. Eximbank & 2,110 & 554 & 2,664 & 2,318 & 516 & 2,833 \\
\hline OPEC Fund & 750 & 210 & 960 & 750 & 268 & 1,018 \\
\hline Trinidad and Tobago & 369 & 465 & 1,034 & 630 & 525 & 1,156 \\
\hline Other & 9 & 25 & 34 & 89 & -- & 89 \\
\hline Commercial. & 13,036 & 13,444 & 26,479 & 15,505 & 15,954 & 31,460 \\
\hline Banco do Brasil & 8,100 & 9.343 & 17,443 & 8,100 & 10,957 & 19.057 \\
\hline Credit Lyonnais & 3,519 & 1.928 & 5.447 & 4,767 & 3,011 & 7,778 \\
\hline Other & 1,417 & 2.172 & 3.589 & 2,638 & 1.986 & 4.625 \\
\hline Government suaranteed & 84,328 & 44,522 & 128,854 & 106,758 & 65,130 & $\underline{171.888}$ \\
\hline Offictal & 1,770 & 1,115 & 2,885 & 2,176 & 1,364 & 3,540 \\
\hline Caribbean Development Bank & 520 & 181 & 701 & 776 & 273 & 1,049 \\
\hline Irinided and Tobago & -- & 539 & 539 & 113 & 624 & 737 \\
\hline Other & 1,250 & 395 & 1.645 & 1,400 & 467 & 1.867 \\
\hline Commerclal & 82,558 & 43,412 & 125,969 & 104,469 & 63.766 & 168,235 \\
\hline ABH Bank (power station and water dist.) & 2,308 & 577 & 2,885 & 3,086 & 914 & 4,000 \\
\hline Cable and Wireless & 745 & 1,795 & 2.540 & 1.745 & 2,789 & 4,534 \\
\hline Ishikawajima-Harima Heavy Industries & 10,412 & 7,540 & 17,952 & 13,387 & 10,523 & 23,910 \\
\hline Ital1 an Institute for Works Abroad & 5,040 & 2.895 & 7,935 & 6,300 & 3,819 & 10,119 \\
\hline Mirlees-Blackstone & 6,659 & 4.149 & 10,808 & 6,461 & 4,843 & 11,305 \\
\hline Morgan-Grenfoll & 47,058 & 19.624 & 66,681 & 59,625 & 31,101 & 90,725 \\
\hline Uitrafin AG & 8,174 & 3,991 & 12,165 & 11,629 & 6.363 & 17,992 \\
\hline Other & 2,162 & 2,841 & 5,003 & 2,236 & 3,414 & 5.650 \\
\hline \multicolumn{7}{|c|}{ (Chenges durtns year) } \\
\hline \multicolumn{7}{|l|}{ Memorandum items } \\
\hline Change in stock of arrears & $\underline{22.785}$ & 18.485 & 41.271 & $\underline{25,688}$ & $\underline{23.512}$ & 49.200 \\
\hline Central Government & $\overline{-5.813}$ & -224 & $-6,037$ & 3.258 & 2,909 & 6.166 \\
\hline Government guaranteed & 28,598 & 18,709 & 47,307 & 22,430 & 20,603 & 43,033 \\
\hline Valuation adjustment $1 /$ & $\frac{8.700}{1.144}$ & $\frac{3.805}{724}$ & $\frac{12,506}{1,868}$ & $\frac{324}{-34}$ & $\frac{747}{-45}$ & $\frac{1.081}{-79}$ \\
\hline $\begin{array}{l}\text { Central Government } \\
\text { Government guaranteed }\end{array}$ & $\begin{array}{l}1,144 \\
7,556\end{array}$ & 3,081 & $\begin{array}{r}1,868 \\
10,638\end{array}$ & $\begin{array}{l}-34 \\
358\end{array}$ & $\begin{array}{l}-45 \\
802\end{array}$ & 1,160 \\
\hline Change in arrears $2 /$ & 14.085 & 14,680 & 28.765 & 25.251 & $\underline{22.755}$ & 48,005 \\
\hline Central Government & $-6,957$ & -947 & $-7,904$ & 3,292 & 2.954 & 6,245 \\
\hline Government suaranteed & 21,042 & 15,628 & 36,670 & 21.959 & 19,801 & 41,760 \\
\hline
\end{tabular}


Table 38. Ant1gua and Barbude: Summary of Arrears Outstanding on External Debt (Concluded)

(In thousands of U,S, dollars)

\begin{tabular}{|c|c|c|c|c|c|c|c|c|c|}
\hline & \multicolumn{3}{|c|}{1992} & \multicolumn{3}{|c|}{1993} & \multicolumn{3}{|c|}{1994} \\
\hline & Principal & Interest & Total & Prine1pal & Interest & Total & Princ1pal & Interest & Total \\
\hline \multicolumn{10}{|c|}{ (Outstandins at end-year) } \\
\hline Total public sector & 146.694 & 106.573 & 253.041 & 157.974 & 125,141 & 283,115 & 179,938 & $\underline{157,166}$ & 337.104 \\
\hline Central Government & 24,665 & $\underline{23,336}$ & 48,001 & 28,203 & 26,208 & 54.312 & 31,904 & $\underline{30,419}$ & $\underline{62,323}$ \\
\hline$\overline{\text { Offlelal }}$ & 6.089 & 4,422 & $\overline{10,511}$ & 6,616 & 4.741 & 11,357 & 7,333 & 5,235 & 12,568 \\
\hline Carlbbean Development Bank & 2,046 & 3,035 & 5,081 & 2,496 & 3.426 & 5,923 & 2,960 & 3,838 & 6,798 \\
\hline U.S. Eximbank & 2,528 & 476 & 3,004 & 2,738 & 435 & 3,172 & 2,948 & 381 & 3,329 \\
\hline OPEC Fund & 750 & 329 & 1,079 & 750 & 394 & $\cdot 1,144$ & 750 & 462 & 1,212 \\
\hline Trinidad and Tobago & 691 & 588 & 1,279 & 550 & 477 & 1,027 & 587 & 520 & 1,107 \\
\hline Other & 74 & -6 & 68 & 82 & 9 & 91 & 89 & 33 & 122 \\
\hline Commercial & 18,576 & 18,914 & 37,490 & 21,587 & 21,367 & 42,954 & 24,571 & 25,184 & 49,755 \\
\hline Banco do Brastl & 8,100 & 12,561 & 20.661 & 8,100 & 14,270 & 22.370 & 8,100 & 16.267 & 24,367 \\
\hline $\begin{array}{l}\text { Credit Lyonnals } \\
\text { Other }\end{array}$ & 6,015 & $\begin{array}{l}4,178 \\
2,174\end{array}$ & $\begin{array}{r}10,193 \\
6,636\end{array}$ & 7.263 & $\begin{array}{l}5,463 \\
1,634\end{array}$ & $\begin{array}{r}12,726 \\
7,858\end{array}$ & $\begin{array}{l}8,145 \\
8,326\end{array}$ & $\begin{array}{l}6,915 \\
2.002\end{array}$ & 15,060 \\
\hline Government guaranteed & 122.029 & 83.237 & 205.040 & 129.770 & 99.033 & 228.556 & 148.034 & 126.747 & 274.781 \\
\hline Officlal & 1,782 & 1,512 & 3,294 & 1.936 & 1,502 & 3,438 & 2,083 & 1,685 & 3,769 \\
\hline Carlbbean Development Bank & 332 & 313 & 645 & 405 & 400 & 805 & 454 & 479 & 933 \\
\hline Trinidad and Tobago & 226 & 711 & 711 & 248 & 585 & 833 & 326 & 643 & 963 \\
\hline Other & 1,449 & 488 & 1,938 & 1,531 & 518 & 2,049 & 1,548 & 564 & 2,111 \\
\hline Commerclel & 120,021 & 81.725 & 201,746 & 127,587 & 97.530 & 225,117 & 145,706 & 125,062 & 270,768 \\
\hline ABN Bank (power station and water dist.) & 3,653 & 1,211 & 4,864 & 3,534 & 1,482 & 5,016 & 3,953 & 2,009 & 5,962 \\
\hline Cable and Wireless & 2.745 & 3,526 & 6,271 & 3,745 & 4,221 & 7,966 & 4.745 & 5,221 & 9,966 \\
\hline Ishikawajima-Harima Heavy Industrles & 16,362 & 13,745 & 30,107 & 19,337 & 17,224 & 36,561 & 22,312 & 20,982 & 43,294 \\
\hline Itali an Institute for Works Abroad & 7,408 & 4,826 & 12,234 & 7,408 & 5,926 & 13,334 & 7,408 & 7,125 & 14,533 \\
\hline Mirlees-Blackstone & 5,222 & 4.627 & 9.850 & 5,116 & 5,286 & 10,402 & 5,397 & 6,432 & 11,829 \\
\hline Morgan-Grenfell & 68,450 & 41,585 & 110,035 & 69.814 & 49,151 & 118,965 & 77,815 & 65,193 & 143,008 \\
\hline Ultrafin $A G$ & 14,310 & 8,491 & 22,801 & 16,794 & 10,033 & 26.826 & 21,934 & 13,247 & 35,181 \\
\hline Other & 1,871 & 3,714 & 5,585 & 1,839 & 4,208 & 6,047 & 2.142 & 4.852 & 6.994 \\
\hline \multicolumn{10}{|c|}{ (Changes during year) } \\
\hline \multicolumn{10}{|l|}{ Memorandum 1tems } \\
\hline Change in outstanding arrears & $\underline{19,153}$ & $\underline{21.525}$ & 40.678 & 11,506 & $\underline{18,568}$ & $\underline{30.074}$ & 22,294 & $\underline{32.025}$ & 54,313 \\
\hline Central Government & 3,768 & 3,418 & 7,186 & 3,538 & 2,773 & 6,311 & 3,701 & 4,311 & 8,011 \\
\hline Government guaranteed & 15,384 & 18,107 & 33.491 & 7,968 & 15,796 & 23,764 & 18,593 & 27.714 & 46,307 \\
\hline Valuation adjustment & -7.056 & $-4,561$ & -11.617 & -6.155 & $-4,367$ & $-10,521$ & 10,954 & 2,998 & $\underline{18.953}$ \\
\hline Central Government & -255 & -410 & -665 & -208 & -189 & -397 & 38 & 55 & 93 \\
\hline Government suaranteed & $-6,801$ & $-4,151$ & $-10,952$ & $-5,946$ & $-4,178$ & $-10,124$ & 10,917 & 7,943 & 18,860 \\
\hline \multicolumn{10}{|l|}{ Change in outstanding arrears } \\
\hline $\begin{array}{l}\text { net of valuation adjustment } \\
\text { Central Government }\end{array}$ & $\frac{25,983}{4,024}$ & $\frac{26.086}{3,827}$ & $\frac{52.069}{7.851}$ & $\frac{17,413}{3,747}$ & $\frac{22,935}{2,962}$ & $\frac{40,348}{6,708}$ & $\frac{11.013}{3,663}$ & $\frac{24,027}{4,256}$ & $\frac{35,040}{7,919}$ \\
\hline Government guaranteed & 21,959 & 22,259 & 44,218 & 13,666 & 19.973 & 33.639 & 7.350 & 19,771 & 27,121 \\
\hline
\end{tabular}

Source: Ministry of Finance.

1/ Including valuation adjustment. 